Supplementary Information for

\title{
Steric Hindrance Favors Sigma Dimerization over Pi Dimerization for Julolidine Dicyanomethyl Radicals
}

\author{
Rui Zhang, Arkady Ellern, Arthur H. Winter \\ 1651 Gilman Hall, Department of Chemistry, Iowa State University, Ames IA 50010
}




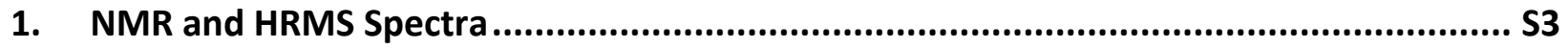

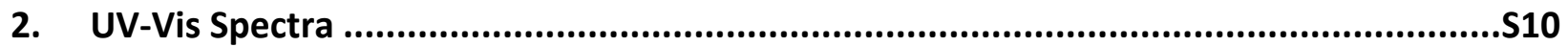

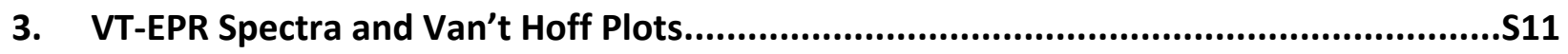

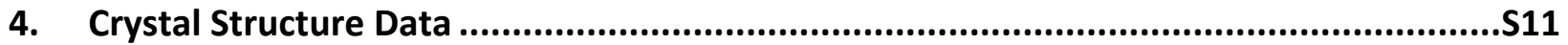

5. Cartesian Coordinates, Energies for Optimized Dimers ................................................S14 


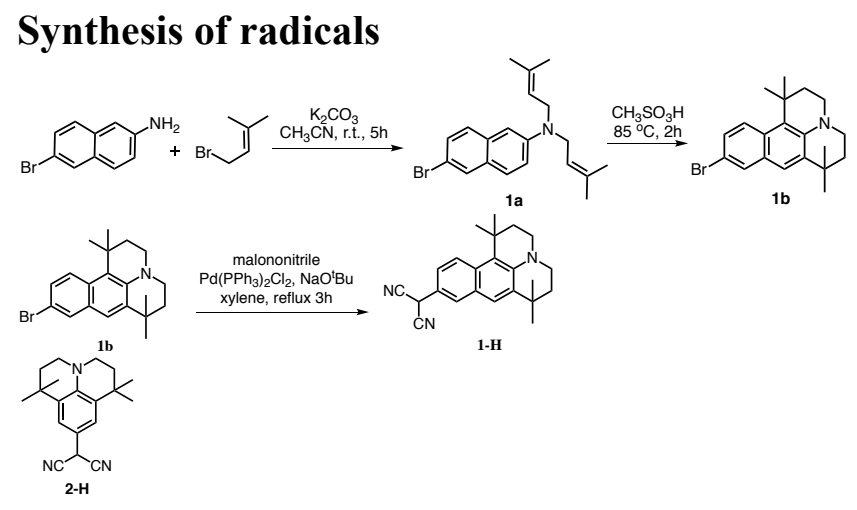

\section{NMR and HRMS Spectra ( ${ }^{1} \mathrm{H}$ NMR $\left.400 \mathrm{MHz},{ }^{13} \mathrm{C} 100 \mathrm{MHz}\right)$}

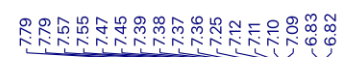

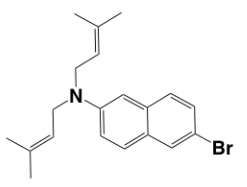

Solvent: $\mathbf{C D C l}_{3}$

1a

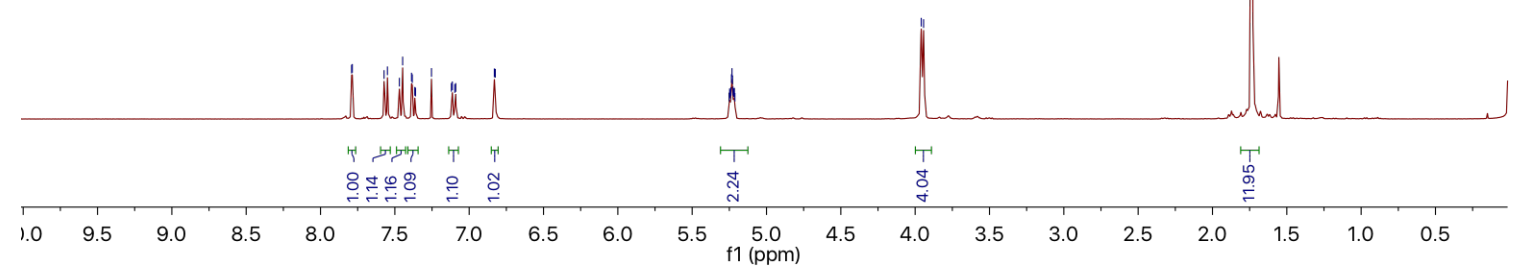




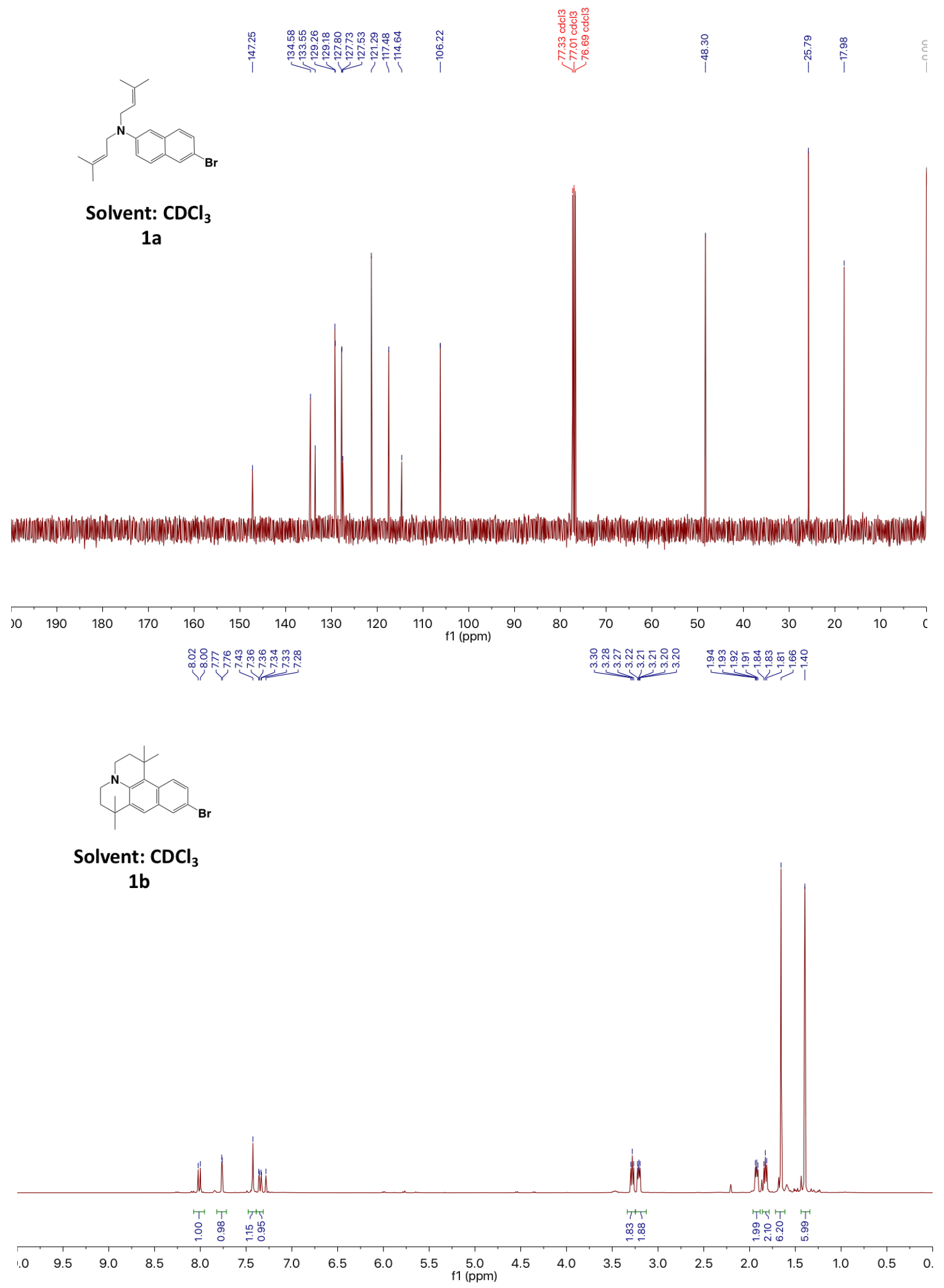




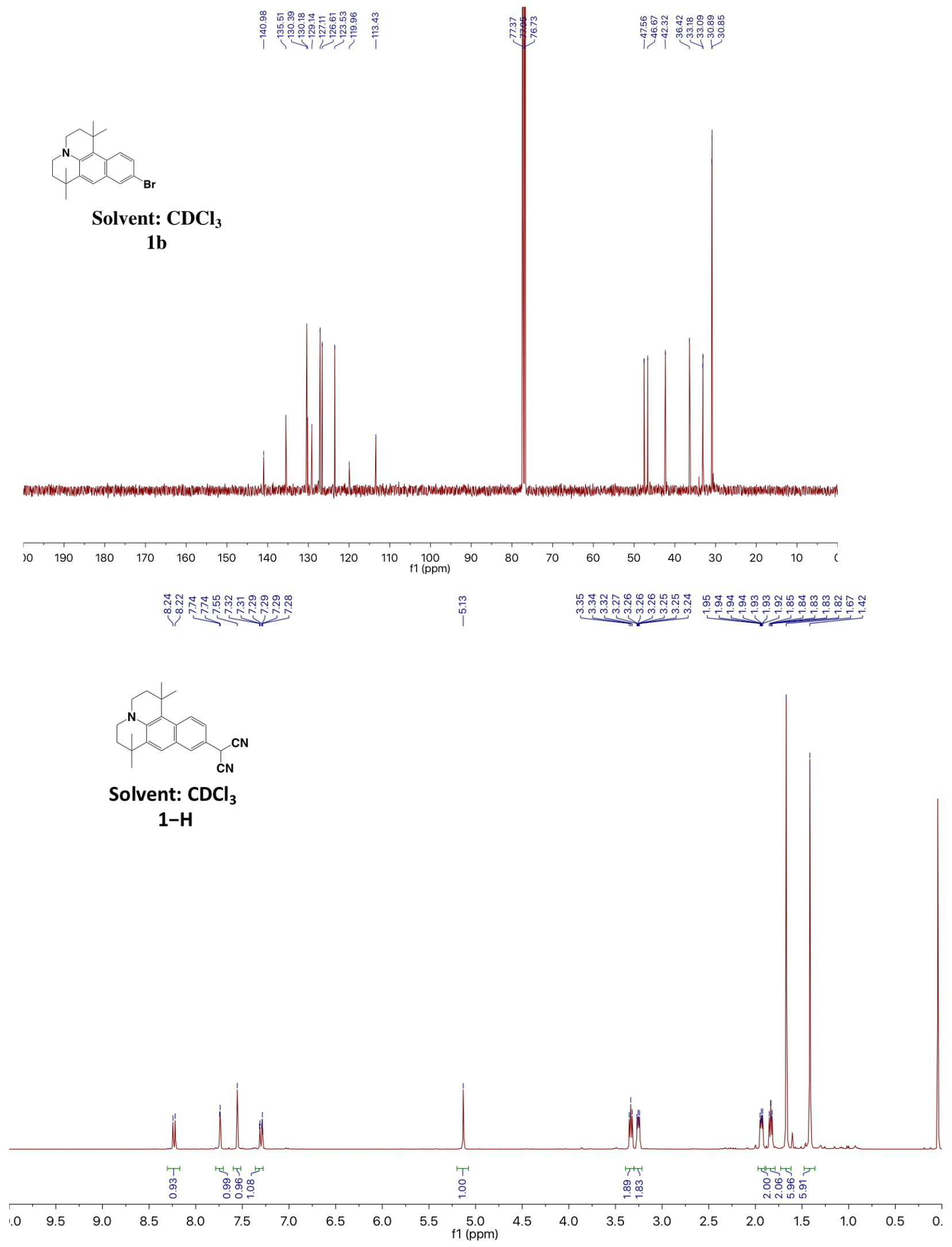




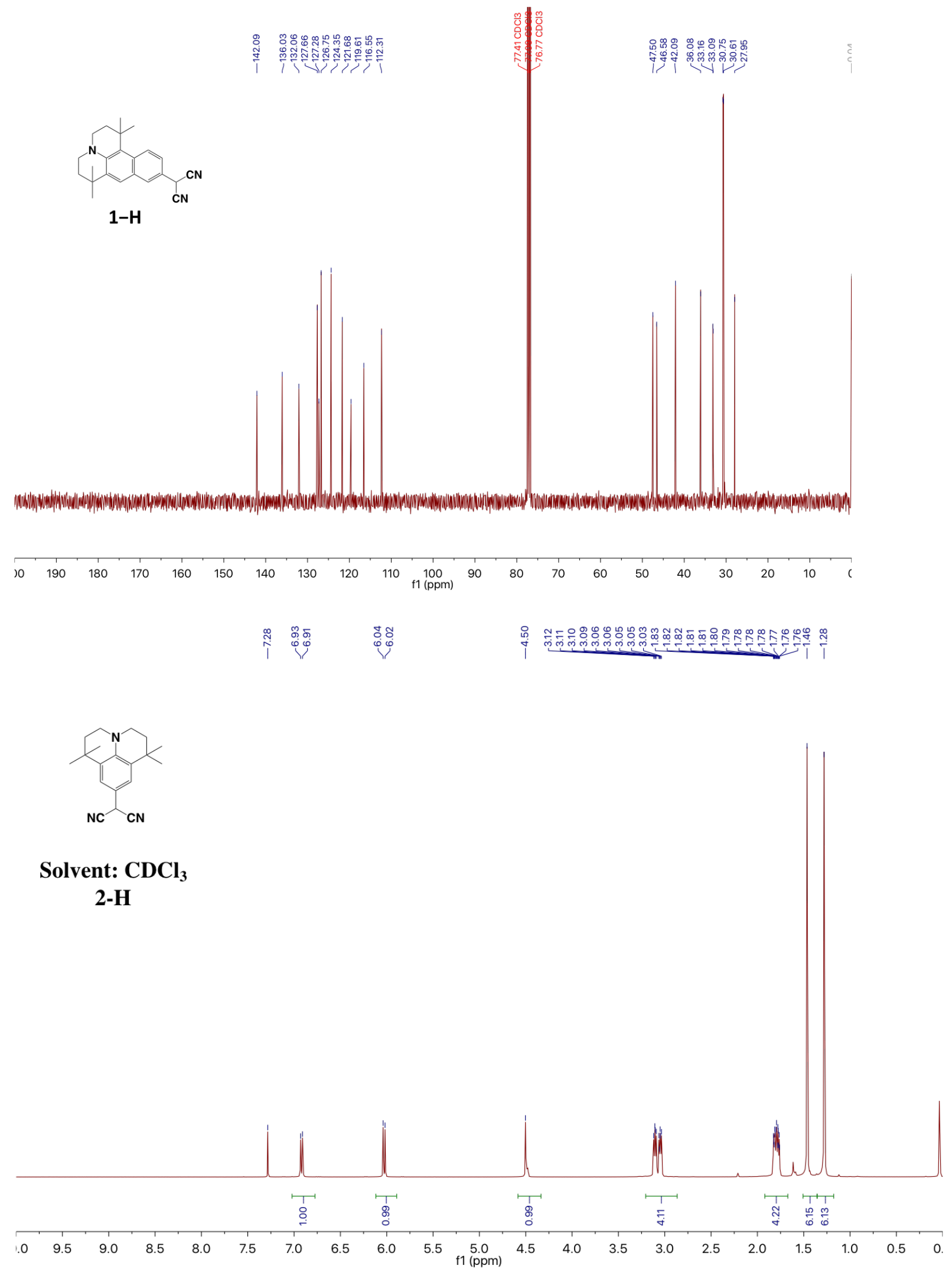




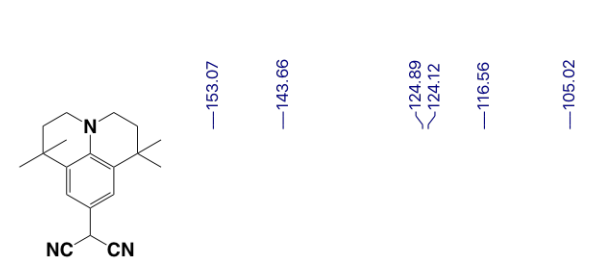

Solvent: $\mathbf{C D C l}_{3}$

2-H

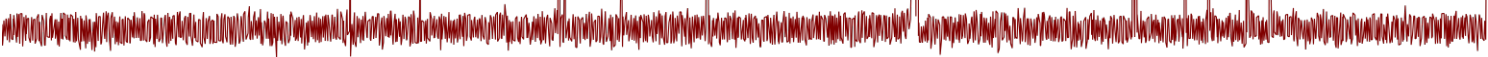

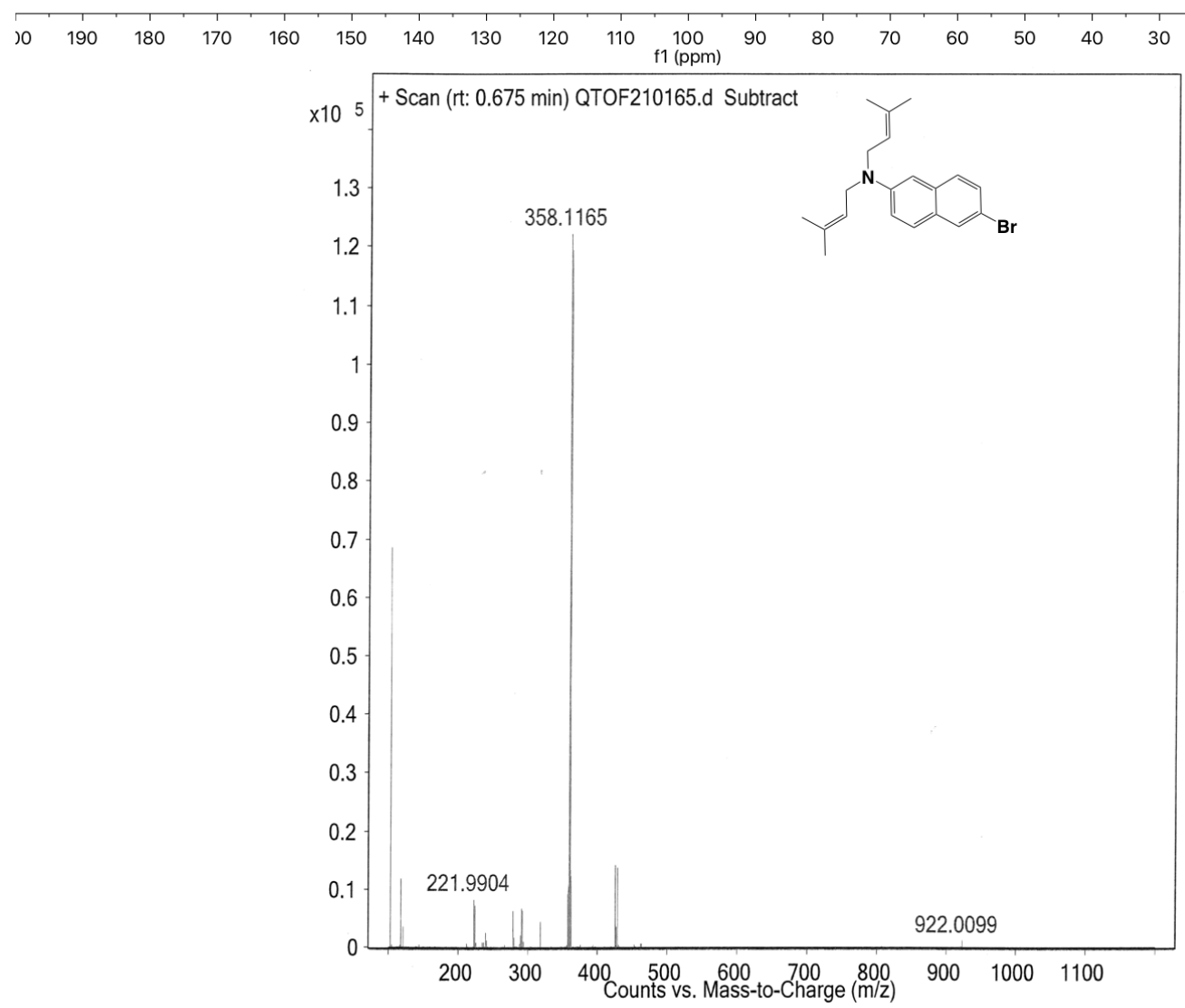



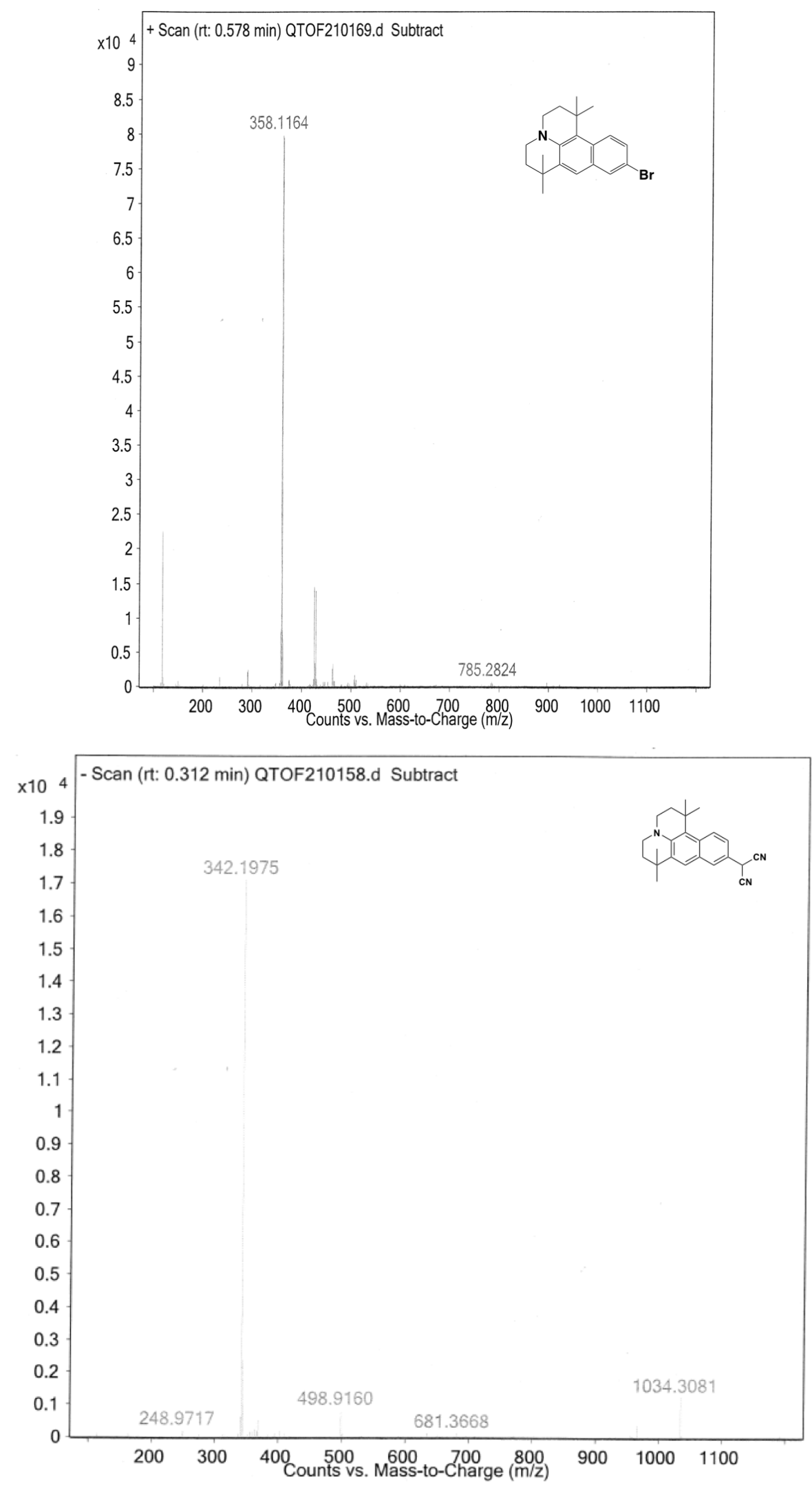


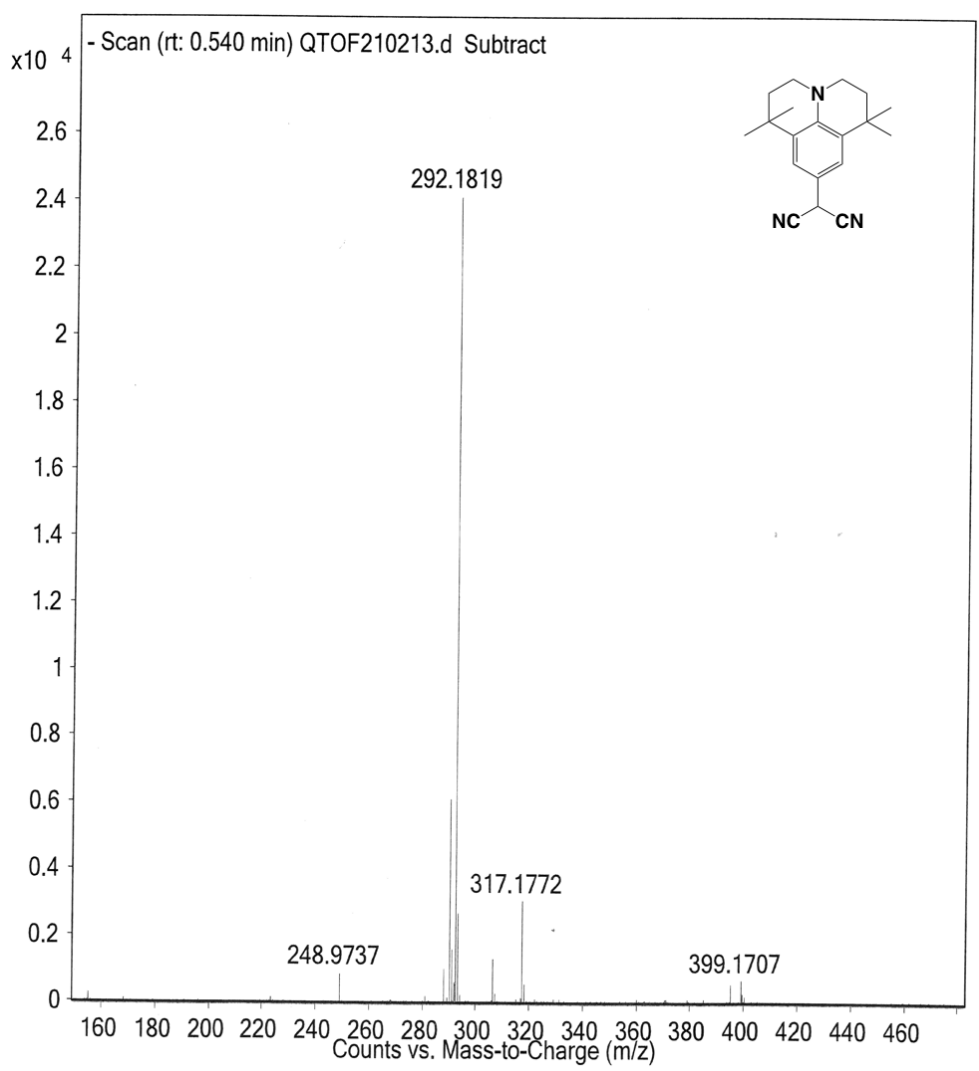




\section{UV-Vis Spectra}
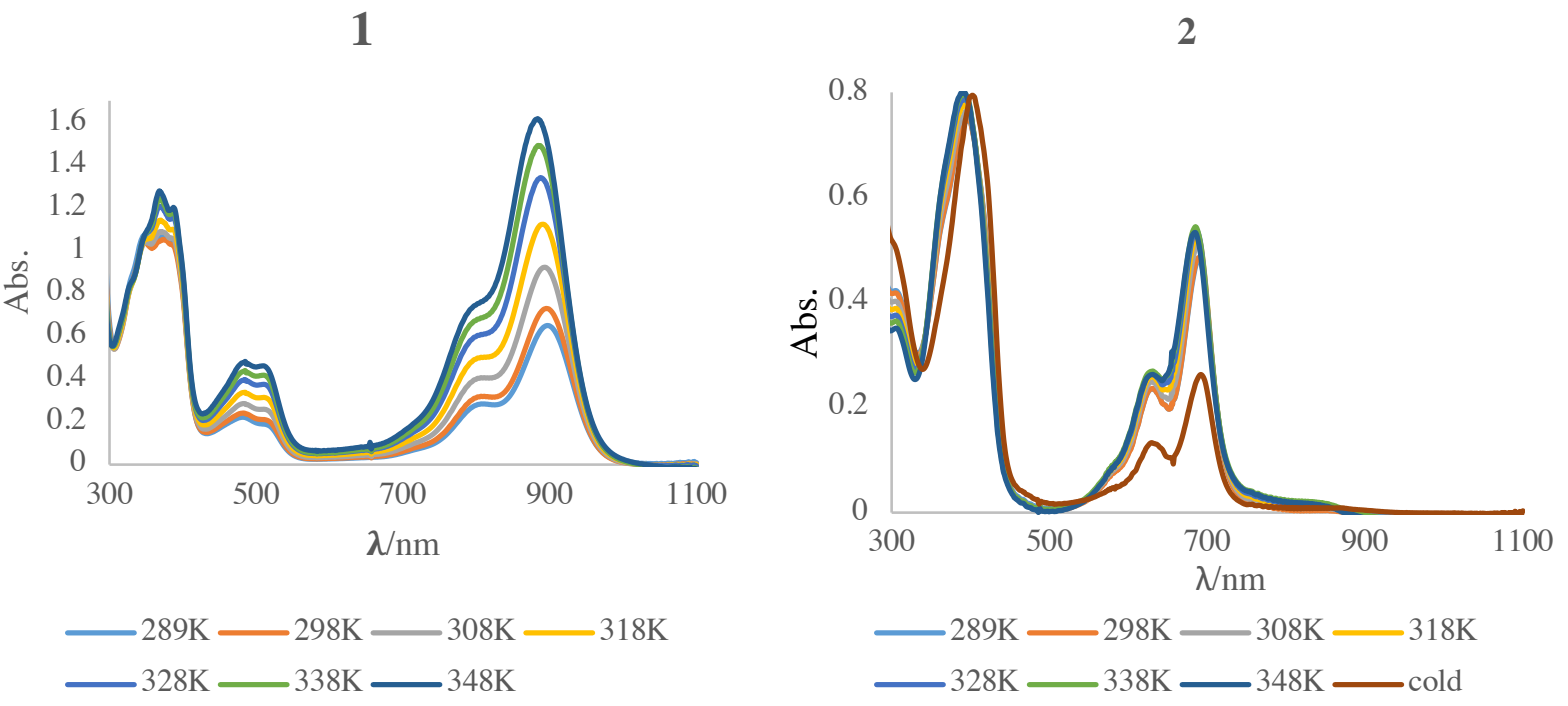

FigureS1. VT-UV-Vis spectra of $\mathbf{1} \bullet$ and $2 \bullet$ in toluene 


\section{VT-EPR Spectra and Van't Hoff Plots}

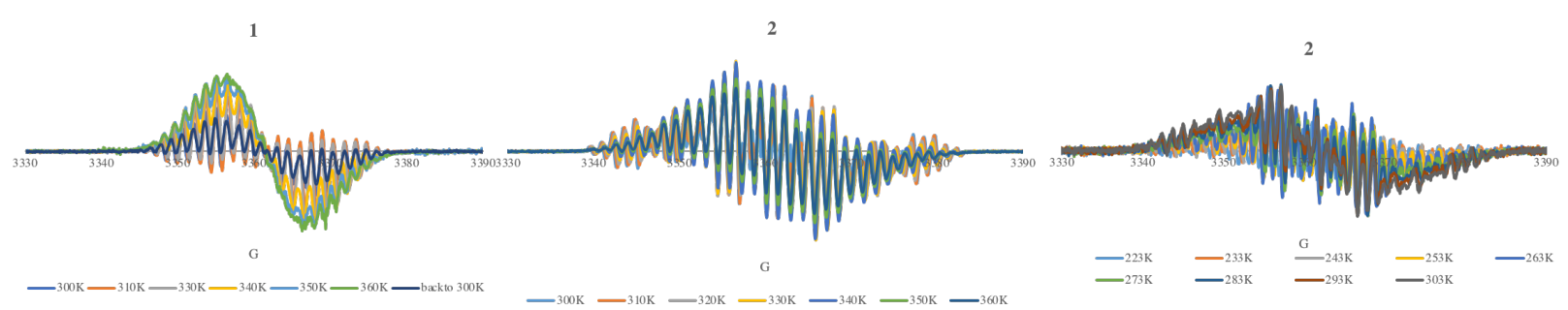

Figure S2. VT-EPR spectra of $4.0 \mathrm{mM} 1 \bullet$ and $3.4 \mathrm{mM} 2 \bullet$ in toluene.
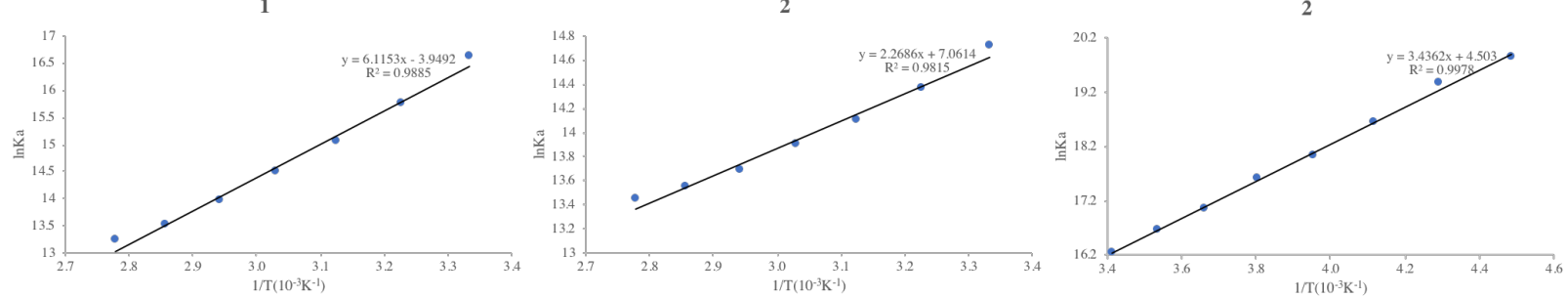

Van't Hoff plots of VT-EPR spectra of $4.0 \mathrm{mM}$ 1• and $3.4 \mathrm{mM} 2 \bullet$.

The dimerization thermodynamic parameters for each of the radicals was determined by variable temperature EPR. From the double integration of each EPR spectra, equilibrium constants were obtained. Intermolecular binding constants were calculated from a van 't Hoff plot. The EPR instrument was calibrated with a TEMPO standard. A van't Hoff plot $\left(\ln K_{\text {eq }}\right.$ vs $\left.1 / T\right)$ provides the equilibrium parameters $\left(\Delta H^{\circ}\right.$ and $\Delta S^{\circ}$, which lead to $\Delta G^{\circ}$ and $K_{\text {a }}$ values $)$ for each system. It is worth noting that EPR spectroscopy is blind to the nature of spin-paired species ( $\sigma$-dimer or pimer) so the $K_{\mathrm{a}}$ value is really measuring the equilibrium between EPR-inactive diamagnetic dimers and spin-unpaired free radicals (or possibly thermally populated triplet excited states of the pimers, at high temperatures). 
Crystal structure data

\section{0\% Thermal Probability Ellipsoids}

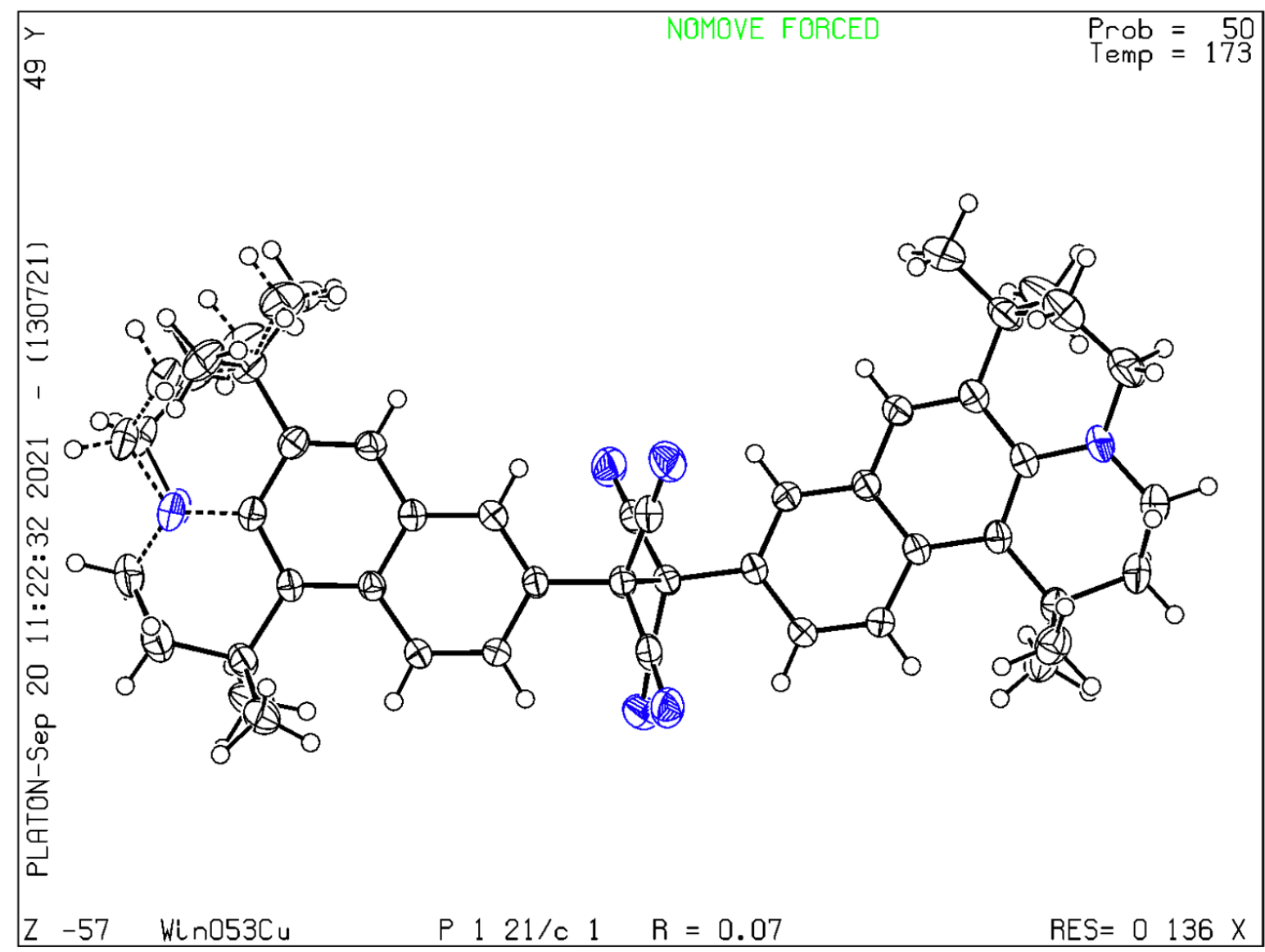

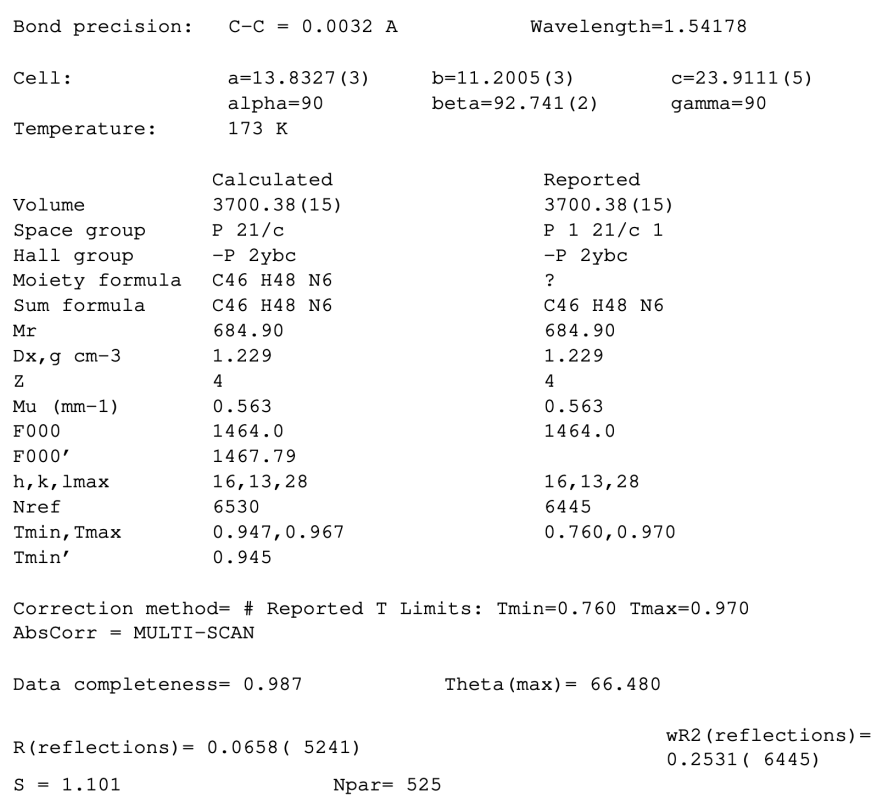




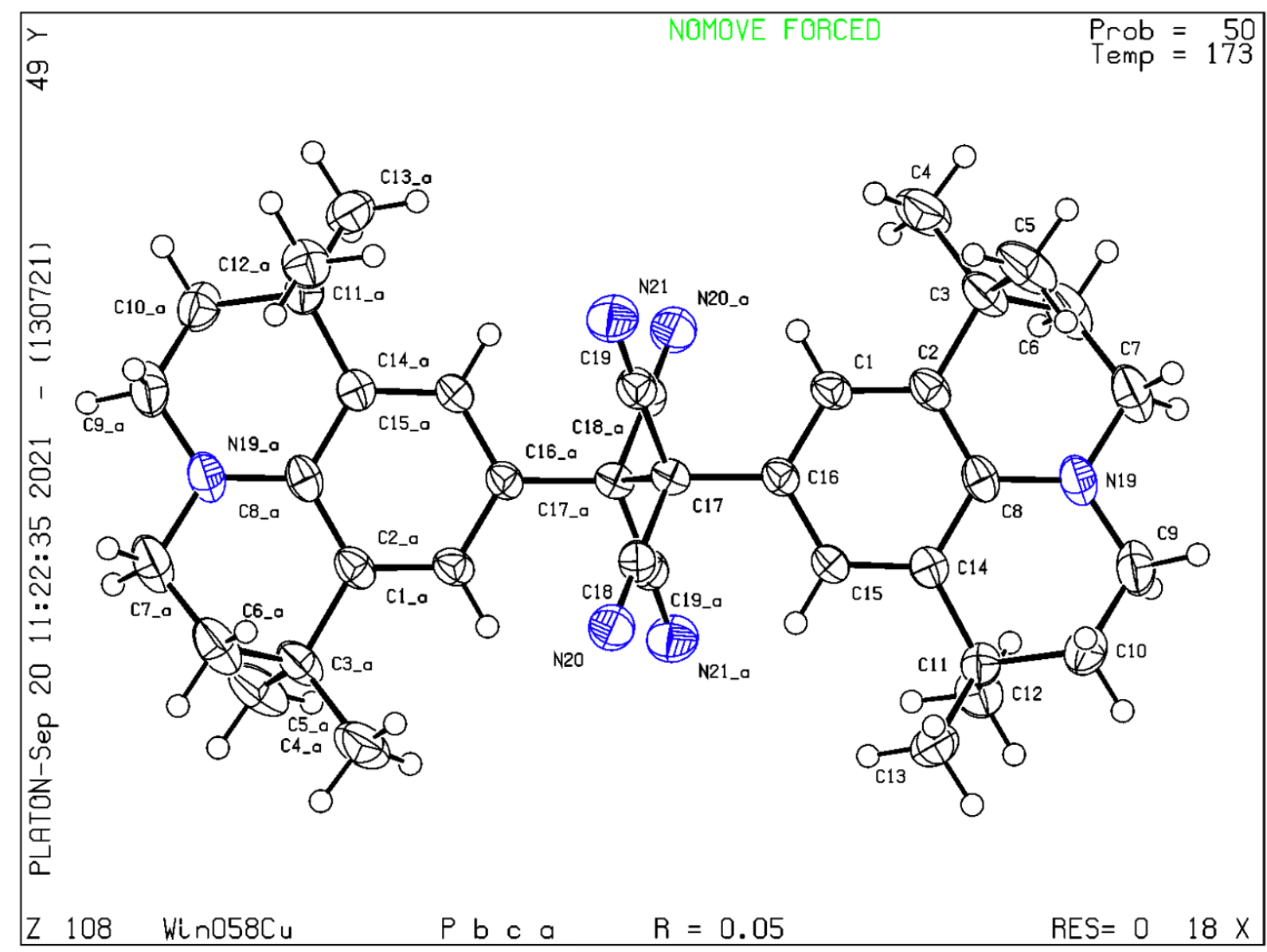

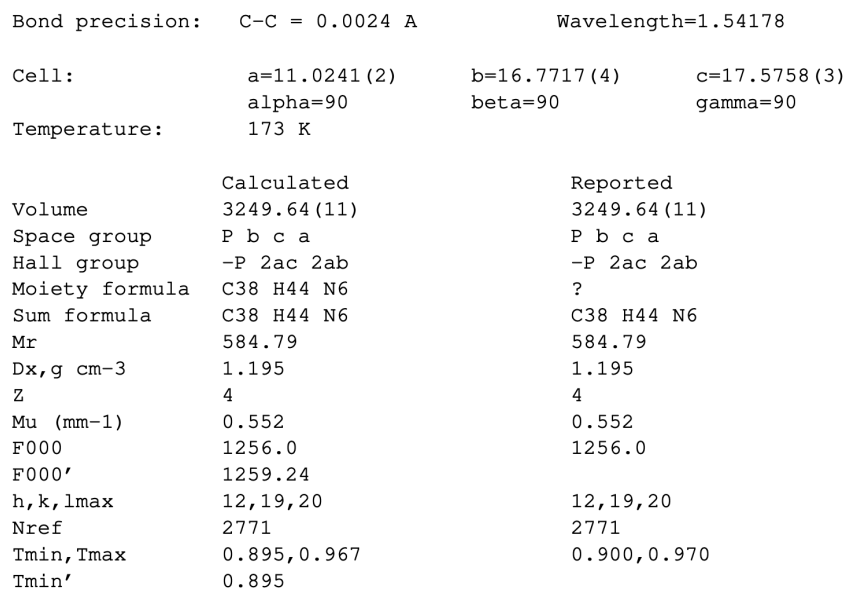

Correction method= \# Reported T Limits: Tmin=0.900 Tmax=0.970

AbsCorr $=$ MULTI-SCAN

Data completeness $=1.000$

Theta $(\max )=65.080$

$\mathrm{R}($ reflections $)=0.0503(2446)$

$\mathrm{S}=1.059$

Npar $=203$ wR2 (reflections $)=$
$0.1662(2771)$ 


\section{Cartesian Coordinates, Energies for Optimized Dimers}

All the structures are optimized at $631+\mathrm{G}(d, p)$ basis level without imaginary frequency.

\begin{tabular}{|c|c|c|c|c|}
\hline & Radical & \multicolumn{3}{|c|}{ Dimer UwB97XD } \\
\hline & \multirow{2}{*}{ UwB97XD/a.u. } & \multicolumn{2}{|c|}{$\pi$-dimer } & \multirow{2}{*}{$\begin{array}{c}\text { sigma-dimer } \\
\mathrm{E}_{\text {sigma }}(\text { unrestricted }) / \text { a.u. }\end{array}$} \\
\hline & & singlet & triplet & \\
\hline 1 & -1054.85182646 & -2109.73413250 & -2109.72856693 & -2109.74169098 \\
\hline 2 & -901.27028391 & -1802.56279368 & -1802.55576292 & -1802.57870538 \\
\hline 3 & -744.03991736 & -1488.12020847 & -1488.10980084 & -1488.11276081 \\
\hline
\end{tabular}

\begin{tabular}{|r|c|c|r|}
\hline \multirow{2}{*}{} & \multicolumn{3}{|c|}{ Dimer UB98 } \\
\cline { 2 - 5 } & singlet & triplet & \multicolumn{1}{|c|}{ sigma-dimer } \\
\hline $\mathbf{1}$ & -2109.50922636 & -2109.50602403 & -2109.51805928 \\
\hline $\mathbf{2}$ & -1802.37879004 & -1802.37843371 & -1802.37380117 \\
\hline $\mathbf{3}$ & -1487.98421024 & -1487.97792452 & -1487.97354491 \\
\hline
\end{tabular}

\begin{tabular}{|r|c|c|r|}
\hline \multirow{2}{*}{} & \multicolumn{3}{|c|}{ Dimer UB97D } \\
\cline { 2 - 5 } & singlet & triplet & \multicolumn{1}{|c|}{ sigma-dimer } \\
\hline $\mathbf{1}$ & -2108.89606900 & -2108.88702214 & -2108.86877878 \\
\hline $\mathbf{2}$ & -1801.85237018 & -1801.84361156 & -1801.83313878 \\
\hline $\mathbf{3}$ & -1487.54190987 & not calculated & -1487.49905634 \\
\hline
\end{tabular}

Table S1. Total energy of optimized radials and dimers.

Julolidine radical $\mathrm{E}(\mathrm{U} \omega \mathrm{B} 97 \mathrm{XD})=-744.03991736$ a.u.

\begin{tabular}{lrrr}
$\begin{array}{c}\text { Atomic } \\
\text { type }\end{array}$ & \multicolumn{3}{c}{ Coordinates(Angstroms) } \\
$\mathrm{N}$ & $\mathrm{X}$ & $\mathrm{Y}$ & $\mathrm{Z}$ \\
$\mathrm{N}$ & 2.577808 & 0.000054 & -0.046925 \\
$\mathrm{~N}$ & -4.360592 & 2.225238 & 0.044885 \\
$\mathrm{C}$ & -4.360372 & -2.225339 & 0.044653 \\
$\mathrm{C}$ & 1.206142 & 0.000016 & -0.077848 \\
$\mathrm{C}$ & 0.481007 & 1.229310 & -0.080835 \\
$\mathrm{C}$ & -0.893136 & 1.209904 & -0.050978 \\
$\mathrm{C}$ & -1.629827 & 0.000019 & -0.032753 \\
$\mathrm{C}$ & -0.893073 & -1.209815 & -0.050759 \\
$\mathrm{C}$ & 0.481080 & -1.229262 & -0.080571 \\
$\mathrm{C}$ & -3.051588 & 0.000009 & 0.006538 \\
$\mathrm{C}$ & -3.788178 & 1.212059 & 0.027955 \\
$\mathrm{C}$ & -3.788186 & -1.212045 & 0.027820 \\
& 1.221931 & 2.545182 & -0.128204
\end{tabular}




$\begin{array}{lrrr}\mathrm{H} & 0.649662 & 3.310330 & 0.404021 \\ \mathrm{H} & 1.301037 & 2.879253 & -1.17184 \\ \mathrm{C} & 2.620002 & 2.403736 & 0.461165 \\ \mathrm{H} & 3.200069 & 3.318290 & 0.309709 \\ \mathrm{H} & 2.558291 & 2.222355 & 1.539949 \\ \mathrm{C} & 3.331477 & 1.236306 & -0.204265 \\ \mathrm{H} & 3.492448 & 1.449504 & -1.273271 \\ \mathrm{H} & 4.315960 & 1.083069 & 0.247827 \\ \mathrm{C} & 3.331260 & -1.236186 & -0.205168 \\ \mathrm{H} & 4.316141 & -1.083135 & 0.246133 \\ \mathrm{H} & 3.491284 & -1.449188 & -1.274344 \\ \mathrm{C} & 2.620347 & -2.403742 & 0.460677 \\ \mathrm{H} & 3.200449 & -3.318189 & 0.308575 \\ \mathrm{H} & 2.559616 & -2.222549 & 1.539540 \\ \mathrm{C} & 1.221754 & -2.545343 & -0.127399 \\ \mathrm{H} & 0.649735 & -3.309750 & 0.406177 \\ \mathrm{H} & 1.299698 & -2.880524 & -1.170095 \\ \mathrm{H} & -1.429290 & -2.155118 & -0.041518 \\ \mathrm{H} & -1.429085 & 2.155092 & -0.042063\end{array}$

Julolidine $\sigma$-dimer $\mathrm{E}(\mathrm{RB} 98)=-1487.97354491$ a.u.

\begin{tabular}{lrrr} 
Atomic & \multicolumn{3}{c}{ Coordinates(Angstroms) } \\
type & $\mathrm{X}$ & $\mathrm{Y}$ & $\mathrm{Z}$ \\
$\mathrm{N}$ & -1.309421 & 5.154158 & 2.983131 \\
$\mathrm{~N}$ & 2.972283 & 0.338213 & -0.118755 \\
$\mathrm{~N}$ & 0.220321 & -1.805942 & 2.377935 \\
$\mathrm{C}$ & -0.968562 & 1.530116 & 2.317890 \\
$\mathrm{H}$ & -1.408769 & 0.599581 & 2.668167 \\
$\mathrm{C}$ & -1.410943 & 2.721607 & 2.886443 \\
$\mathrm{C}$ & -2.476775 & 2.725381 & 3.962292 \\
$\mathrm{C}$ & -3.337980 & 3.989144 & 3.829928 \\
$\mathrm{H}$ & -3.872803 & 3.969486 & 2.871516 \\
$\mathrm{H}$ & -4.088525 & 4.043373 & 4.626990 \\
$\mathrm{C}$ & -2.446982 & 5.230548 & 3.899336 \\
$\mathrm{H}$ & -3.031521 & 6.124599 & 3.638190 \\
$\mathrm{H}$ & -2.083738 & 5.376417 & 4.931615 \\
$\mathrm{C}$ & -0.869996 & 3.960721 & 2.441099 \\
$\mathrm{C}$ & -0.685982 & 6.437500 & 2.664833 \\
$\mathrm{H}$ & -0.662998 & 7.038735 & 3.585181 \\
$\mathrm{H}$ & -1.310913 & 6.986915 & 1.939126
\end{tabular}




\begin{tabular}{|c|c|c|c|}
\hline $\mathrm{C}$ & 0.731780 & 6.275087 & 2.113859 \\
\hline $\mathrm{H}$ & 1.399388 & 5.922003 & 2.910781 \\
\hline $\mathrm{H}$ & 1.100592 & 7.252349 & 1.781325 \\
\hline $\mathrm{C}$ & 0.725469 & 5.261755 & 0.961015 \\
\hline $\mathrm{C}$ & 0.137326 & 3.949030 & 1.435299 \\
\hline $\mathrm{C}$ & 0.576506 & 2.735619 & 0.911929 \\
\hline $\mathrm{H}$ & 1.358247 & 2.755736 & 0.156317 \\
\hline $\mathrm{C}$ & 0.026215 & 1.513194 & 1.327864 \\
\hline $\mathrm{C}$ & 0.456301 & 0.195110 & 0.670807 \\
\hline $\mathrm{C}$ & 1.868176 & 0.258196 & 0.231191 \\
\hline $\mathrm{C}$ & 0.336882 & -0.934310 & 1.619929 \\
\hline $\mathrm{N}$ & 1.309421 & -5.154158 & -2.983131 \\
\hline $\mathrm{N}$ & -2.972283 & -0.338213 & 0.118755 \\
\hline $\mathrm{N}$ & -0.220321 & 1.805942 & -2.377935 \\
\hline $\mathrm{C}$ & 0.968562 & -1.530116 & -2.317890 \\
\hline $\mathrm{H}$ & 1.408769 & -0.599581 & -2.668167 \\
\hline $\mathrm{C}$ & 1.410943 & -2.721607 & -2.886443 \\
\hline $\mathrm{C}$ & 2.476775 & -2.725381 & -3.962292 \\
\hline $\mathrm{C}$ & 3.337980 & -3.989144 & -3.829928 \\
\hline $\mathrm{H}$ & 3.872803 & -3.969486 & -2.871516 \\
\hline $\mathrm{H}$ & 4.088525 & -4.043373 & -4.626990 \\
\hline $\mathrm{C}$ & 2.446982 & -5.230548 & -3.899336 \\
\hline $\mathrm{H}$ & 3.031521 & -6.124599 & -3.638190 \\
\hline $\mathrm{H}$ & 2.083738 & -5.376417 & -4.931615 \\
\hline $\mathrm{C}$ & 0.869996 & -3.960721 & -2.441099 \\
\hline $\mathrm{C}$ & 0.685982 & -6.437500 & -2.664833 \\
\hline $\mathrm{H}$ & 0.662998 & -7.038735 & -3.585181 \\
\hline $\mathrm{H}$ & 1.310913 & -6.986915 & -1.939126 \\
\hline $\mathrm{C}$ & -0.731780 & -6.275087 & -2.113859 \\
\hline $\mathrm{H}$ & -1.399388 & -5.922003 & -2.910781 \\
\hline $\mathrm{H}$ & -1.100592 & -7.252349 & -1.781325 \\
\hline $\mathrm{C}$ & -0.725469 & -5.261755 & -0.961015 \\
\hline $\mathrm{C}$ & -0.137326 & -3.949030 & -1.435299 \\
\hline $\mathrm{C}$ & -0.576506 & -2.735619 & -0.911929 \\
\hline $\mathrm{H}$ & -1.358247 & -2.755736 & -0.156317 \\
\hline $\mathrm{C}$ & -0.026215 & -1.513194 & -1.327864 \\
\hline $\mathrm{C}$ & -0.456301 & -0.195110 & -0.670807 \\
\hline $\mathrm{C}$ & -1.868176 & -0.258196 & -0.231191 \\
\hline $\mathrm{C}$ & -0.336882 & 0.934310 & -1.619929 \\
\hline $\mathrm{H}$ & 1.738340 & 5.101329 & 0.573894 \\
\hline
\end{tabular}




$\begin{array}{lrrr}\mathrm{H} & 0.128327 & 5.662593 & 0.127502 \\ \mathrm{H} & -2.010500 & 2.706888 & 4.959674 \\ \mathrm{H} & -3.092236 & 1.821960 & 3.882922 \\ \mathrm{H} & -0.128327 & -5.662593 & -0.127502 \\ \mathrm{H} & -1.738340 & -5.101329 & -0.573894 \\ \mathrm{H} & 3.092236 & -1.821960 & -3.882922 \\ \mathrm{H} & 2.010500 & -2.706888 & -4.959674\end{array}$

Julolidine $\sigma$-dimer $\mathrm{E}(\mathrm{RB} 97 \mathrm{D})=-1487.49905634$ a.u.

\begin{tabular}{lrrr} 
Atomic & \multicolumn{3}{c}{ Coordinates(Angstroms) } \\
type & $\mathrm{X}$ & $\mathrm{Y}$ & $\mathrm{Z}$ \\
$\mathrm{N}$ & -1.304172 & 5.125815 & 2.960311 \\
$\mathrm{~N}$ & 2.976033 & 0.247075 & -0.229202 \\
$\mathrm{~N}$ & 0.199493 & -1.914946 & 2.295818 \\
$\mathrm{C}$ & -0.910639 & 1.489536 & 2.354892 \\
$\mathrm{H}$ & -1.352357 & 0.555782 & 2.703594 \\
$\mathrm{C}$ & -1.371482 & 2.685126 & 2.904974 \\
$\mathrm{C}$ & -2.451795 & 2.694101 & 3.966844 \\
$\mathrm{C}$ & -3.336772 & 3.941161 & 3.787692 \\
$\mathrm{H}$ & -3.841071 & 3.892848 & 2.809864 \\
$\mathrm{H}$ & -4.109446 & 3.999988 & 4.568055 \\
$\mathrm{C}$ & -2.465963 & 5.201778 & 3.854060 \\
$\mathrm{H}$ & -3.058891 & 6.085630 & 3.561824 \\
$\mathrm{H}$ & -2.120954 & 5.369168 & 4.893435 \\
$\mathrm{C}$ & -0.840387 & 3.928049 & 2.442673 \\
$\mathrm{C}$ & -0.688002 & 6.416620 & 2.634326 \\
$\mathrm{H}$ & -0.680160 & 7.027844 & 3.553283 \\
$\mathrm{H}$ & -1.315436 & 6.949097 & 1.892514 \\
$\mathrm{C}$ & 0.738977 & 6.260065 & 2.094867 \\
$\mathrm{H}$ & 1.400327 & 5.910751 & 2.903566 \\
$\mathrm{H}$ & 1.102485 & 7.241127 & 1.755916 \\
$\mathrm{C}$ & 0.744622 & 5.232519 & 0.948643 \\
$\mathrm{C}$ & 0.179089 & 3.916452 & 1.442043 \\
$\mathrm{C}$ & 0.639766 & 2.700170 & 0.938986 \\
$\mathrm{H}$ & 1.420566 & 2.717939 & 0.178167 \\
$\mathrm{C}$ & 0.100081 & 1.472566 & 1.372922 \\
$\mathrm{C}$ & 0.501666 & 0.169870 & 0.698511 \\
$\mathrm{C}$ & 1.886901 & 0.195060 & 0.195370 \\
$\mathrm{C}$ & 0.355457 & -0.997341 & 1.586737 \\
$\mathrm{~N}$ & 1.304172 & -5.125815 & -2.960311
\end{tabular}




\begin{tabular}{lrrr}
$\mathrm{N}$ & -2.976033 & -0.247075 & 0.229202 \\
$\mathrm{~N}$ & -0.199493 & 1.914946 & -2.295818 \\
$\mathrm{C}$ & 0.910639 & -1.489536 & -2.354892 \\
$\mathrm{H}$ & 1.352357 & -0.555782 & -2.703594 \\
$\mathrm{C}$ & 1.371482 & -2.685126 & -2.904974 \\
$\mathrm{C}$ & 2.451795 & -2.694101 & -3.966844 \\
$\mathrm{C}$ & 3.336772 & -3.941161 & -3.787692 \\
$\mathrm{H}$ & 3.841071 & -3.892848 & -2.809864 \\
$\mathrm{H}$ & 4.109446 & -3.999988 & -4.568055 \\
$\mathrm{C}$ & 2.465963 & -5.201778 & -3.854060 \\
$\mathrm{H}$ & 3.058891 & -6.085630 & -3.561824 \\
$\mathrm{H}$ & 2.120954 & -5.369168 & -4.893435 \\
$\mathrm{C}$ & 0.840387 & -3.928049 & -2.442673 \\
$\mathrm{C}$ & 0.688002 & -6.416620 & -2.634326 \\
$\mathrm{H}$ & 0.680160 & -7.027844 & -3.553283 \\
$\mathrm{H}$ & 1.315436 & -6.949097 & -1.892514 \\
$\mathrm{C}$ & -0.738977 & -6.260065 & -2.094867 \\
$\mathrm{H}$ & -1.400327 & -5.910751 & -2.903566 \\
$\mathrm{H}$ & -1.102485 & -7.241127 & -1.755916 \\
$\mathrm{C}$ & -0.744622 & -5.232519 & -0.948643 \\
$\mathrm{C}$ & -0.179089 & -3.916452 & -1.442043 \\
$\mathrm{C}$ & -0.639766 & -2.700170 & -0.938986 \\
$\mathrm{H}$ & -1.420566 & -2.717939 & -0.178167 \\
$\mathrm{C}$ & -0.100081 & -1.472566 & -1.372922 \\
$\mathrm{C}$ & -0.501666 & -0.169870 & -0.698511 \\
$\mathrm{C}$ & -1.886901 & -0.195060 & -0.195370 \\
$\mathrm{C}$ & -0.355457 & 0.997341 & -1.586737 \\
$\mathrm{H}$ & 1.760281 & 5.082395 & 0.554077 \\
$\mathrm{H}$ & 0.128658 & 5.618465 & 0.116510 \\
$\mathrm{H}$ & -1.997060 & 2.719502 & 4.973817 \\
$\mathrm{H}$ & -3.048513 & 1.772427 & 3.902648 \\
$\mathrm{H}$ & -0.128658 & -5.618465 & -0.116510 \\
$\mathrm{H}$ & -1.760281 & -5.082395 & -0.554077 \\
$\mathrm{H}$ & 3.048513 & -1.772427 & -3.902648 \\
$\mathrm{H}$ & 1.997060 & -2.719502 & -4.973817 \\
& & & \\
\hline
\end{tabular}

Julolidine $\sigma$-dimer $\mathrm{E}(\mathrm{U} \omega \mathrm{B} 97 \mathrm{XD})=-1488.11276081$ a.u.

Atomic Coordinates(Angstroms)

$\begin{array}{lccc}\text { type } & \mathrm{X} & \mathrm{Y} & \mathrm{Z} \\ \mathrm{N} & -1.310992 & 5.116714 & 2.941952\end{array}$




\begin{tabular}{|c|c|c|c|}
\hline $\mathrm{N}$ & 2.955310 & 0.325096 & -0.150195 \\
\hline $\mathrm{N}$ & 0.211848 & -1.812901 & 2.351962 \\
\hline $\mathrm{C}$ & -0.961242 & 1.509116 & 2.292905 \\
\hline $\mathrm{H}$ & -1.400564 & 0.579178 & 2.643504 \\
\hline $\mathrm{C}$ & -1.407468 & 2.695420 & 2.853057 \\
\hline $\mathrm{C}$ & -2.473102 & 2.699095 & 3.919309 \\
\hline $\mathrm{C}$ & -3.323399 & 3.960184 & 3.790236 \\
\hline $\mathrm{H}$ & -3.851050 & 3.948430 & 2.829828 \\
\hline $\mathrm{H}$ & -4.076406 & 4.013096 & 4.581680 \\
\hline $\mathrm{C}$ & -2.429676 & 5.188930 & 3.868236 \\
\hline $\mathrm{H}$ & -3.009404 & 6.089068 & 3.626831 \\
\hline $\mathrm{H}$ & -2.054078 & 5.315433 & 4.896756 \\
\hline $\mathrm{C}$ & -0.870228 & 3.925681 & 2.407916 \\
\hline $\mathrm{C}$ & -0.663182 & 6.389281 & 2.663814 \\
\hline $\mathrm{H}$ & -0.612285 & 6.955197 & 3.603248 \\
\hline $\mathrm{H}$ & -1.283258 & 6.977373 & 1.968767 \\
\hline $\mathrm{C}$ & 0.736026 & 6.215997 & 2.090874 \\
\hline $\mathrm{H}$ & 1.410613 & 5.838820 & 2.868037 \\
\hline $\mathrm{H}$ & 1.114283 & 7.190845 & 1.769626 \\
\hline $\mathrm{C}$ & 0.695950 & 5.226822 & 0.928707 \\
\hline $\mathrm{C}$ & 0.127222 & 3.915523 & 1.405198 \\
\hline $\mathrm{C}$ & 0.571061 & 2.709009 & 0.888751 \\
\hline $\mathrm{H}$ & 1.347967 & 2.729547 & 0.129531 \\
\hline $\mathrm{C}$ & 0.030148 & 1.494567 & 1.312000 \\
\hline $\mathrm{C}$ & 0.452781 & 0.182671 & 0.652768 \\
\hline $\mathrm{C}$ & 1.859742 & 0.245150 & 0.210572 \\
\hline $\mathrm{C}$ & 0.335554 & -0.943040 & 1.600395 \\
\hline $\mathrm{N}$ & 1.310992 & -5.116714 & -2.941952 \\
\hline $\mathrm{N}$ & -2.955310 & -0.325096 & 0.150195 \\
\hline $\mathrm{N}$ & -0.211848 & 1.812901 & -2.351962 \\
\hline $\mathrm{C}$ & 0.961242 & -1.509116 & -2.292905 \\
\hline $\mathrm{H}$ & 1.400564 & -0.579178 & -2.643504 \\
\hline $\mathrm{C}$ & 1.407468 & -2.695420 & -2.853057 \\
\hline $\mathrm{C}$ & 2.473102 & -2.699095 & -3.919309 \\
\hline $\mathrm{C}$ & 3.323399 & -3.960184 & -3.790236 \\
\hline $\mathrm{H}$ & 3.851050 & -3.948430 & -2.829828 \\
\hline $\mathrm{H}$ & 4.076406 & -4.013096 & -4.581680 \\
\hline $\mathrm{C}$ & 2.429676 & -5.188930 & -3.868236 \\
\hline $\mathrm{H}$ & 3.009404 & -6.089068 & -3.626831 \\
\hline $\mathrm{H}$ & 2.054078 & -5.315433 & -4.896756 \\
\hline
\end{tabular}




$\begin{array}{lrrr}\mathrm{C} & 0.870228 & -3.925681 & -2.407916 \\ \mathrm{C} & 0.663182 & -6.389281 & -2.663814 \\ \mathrm{H} & 0.612285 & -6.955197 & -3.603248 \\ \mathrm{H} & 1.283258 & -6.977373 & -1.968767 \\ \mathrm{C} & -0.736026 & -6.215997 & -2.090874 \\ \mathrm{H} & -1.410613 & -5.838820 & -2.868037 \\ \mathrm{H} & -1.114283 & -7.190845 & -1.769626 \\ \mathrm{C} & -0.695950 & -5.226822 & -0.928707 \\ \mathrm{C} & -0.127222 & -3.915523 & -1.405198 \\ \mathrm{C} & -0.571061 & -2.709009 & -0.888751 \\ \mathrm{H} & -1.347967 & -2.729547 & -0.129531 \\ \mathrm{C} & -0.030148 & -1.494567 & -1.312000 \\ \mathrm{C} & -0.452781 & -0.182671 & -0.652768 \\ \mathrm{C} & -1.859742 & -0.245150 & -0.210572 \\ \mathrm{C} & -0.335554 & 0.943040 & -1.600395 \\ \mathrm{H} & 1.693639 & 5.074197 & 0.506886 \\ \mathrm{H} & 0.069035 & 5.634597 & 0.124215 \\ \mathrm{H} & -2.008994 & 2.673604 & 4.914640 \\ \mathrm{H} & -3.090986 & 1.800525 & 3.832672 \\ \mathrm{H} & -0.069035 & -5.634597 & -0.124215 \\ \mathrm{H} & -1.693639 & -5.074197 & -0.506886 \\ \mathrm{H} & 3.090986 & -1.800525 & -3.832672 \\ \mathrm{H} & 2.008994 & -2.673604 & -4.914640\end{array}$

Julolidine $\pi$-dimer singlet $\mathrm{E}(\mathrm{U} \omega \mathrm{B} 97 \mathrm{XD})=-1488.12020847$ a.u.; triplet $\mathrm{E}(\mathrm{U} \omega \mathrm{B} 97 \mathrm{XD})=$ 1488.10980084 a.u.

\begin{tabular}{lrrr} 
Atomic & \multicolumn{3}{c}{ Coordinates(Angstroms) } \\
type & $\mathrm{X}$ & $\mathrm{Y}$ & $\mathrm{Z}$ \\
$\mathrm{N}$ & 4.106971 & 0.000330 & -0.372964 \\
$\mathrm{~N}$ & -2.451445 & -2.208753 & -2.598256 \\
$\mathrm{~N}$ & -2.450607 & 2.214118 & -2.595277 \\
$\mathrm{C}$ & 2.791925 & 0.000649 & -0.700668 \\
$\mathrm{C}$ & 2.096506 & -1.238218 & -0.925600 \\
$\mathrm{C}$ & 0.784459 & -1.213213 & -1.315113 \\
$\mathrm{C}$ & 0.072943 & 0.001489 & -1.529801 \\
$\mathrm{C}$ & 0.784650 & 1.215803 & -1.313143 \\
$\mathrm{C}$ & 2.096666 & 1.240011 & -0.923617 \\
$\mathrm{C}$ & -1.263494 & 0.002041 & -1.983626 \\
$\mathrm{C}$ & -1.927918 & -1.205435 & -2.323579 \\
$\mathrm{C}$ & -1.927474 & 1.210247 & -2.321879
\end{tabular}




\begin{tabular}{|c|c|c|c|}
\hline $\mathrm{C}$ & 2.811723 & -2.552906 & -0.742233 \\
\hline $\mathrm{H}$ & 2.414123 & -3.285486 & -1.450661 \\
\hline $\mathrm{H}$ & 2.613957 & -2.933533 & 0.267420 \\
\hline $\mathrm{C}$ & 4.316391 & -2.387661 & -0.921186 \\
\hline $\mathrm{H}$ & 4.839849 & -3.304685 & -0.638101 \\
\hline $\mathrm{H}$ & 4.556128 & -2.175882 & -1.969749 \\
\hline $\mathrm{C}$ & 4.807471 & -1.241959 & -0.051122 \\
\hline $\mathrm{H}$ & 4.669458 & -1.480228 & 1.011643 \\
\hline $\mathrm{H}$ & 5.873495 & -1.066302 & -0.218603 \\
\hline $\mathrm{C}$ & 4.807900 & 1.241986 & -0.049721 \\
\hline $\mathrm{H}$ & 5.873810 & 1.066344 & -0.218060 \\
\hline $\mathrm{H}$ & 4.670542 & 1.478761 & 1.013469 \\
\hline $\mathrm{C}$ & 4.316670 & 2.389096 & -0.917838 \\
\hline $\mathrm{H}$ & 4.840405 & 3.305579 & -0.633502 \\
\hline $\mathrm{H}$ & 4.556009 & 2.178910 & -1.966817 \\
\hline $\mathrm{C}$ & 2.812111 & 2.554303 & -0.738146 \\
\hline $\mathrm{H}$ & 2.414401 & 3.288098 & -1.445246 \\
\hline $\mathrm{H}$ & 2.614662 & 2.933214 & 0.272208 \\
\hline $\mathrm{H}$ & 0.276277 & 2.158684 & -1.495371 \\
\hline $\mathrm{H}$ & 0.276056 & -2.155819 & -1.498841 \\
\hline $\mathrm{N}$ & -4.106969 & -0.000217 & 0.372908 \\
\hline $\mathrm{N}$ & 2.451685 & 2.208486 & 2.598312 \\
\hline $\mathrm{N}$ & 2.450622 & -2.214250 & 2.595283 \\
\hline $\mathrm{C}$ & -2.791937 & -0.000597 & 0.700671 \\
\hline $\mathrm{C}$ & -2.096472 & 1.238237 & 0.925620 \\
\hline $\mathrm{C}$ & -0.784440 & 1.213170 & 1.315183 \\
\hline $\mathrm{C}$ & -0.072994 & -0.001565 & 1.529912 \\
\hline $\mathrm{C}$ & -0.784745 & -1.215845 & 1.313228 \\
\hline $\mathrm{C}$ & -2.096746 & -1.239992 & 0.923645 \\
\hline $\mathrm{C}$ & 1.263448 & -0.002174 & 1.983731 \\
\hline $\mathrm{C}$ & 1.927979 & 1.205276 & 2.323582 \\
\hline $\mathrm{C}$ & 1.927431 & -1.210411 & 2.321875 \\
\hline $\mathrm{C}$ & -2.811619 & 2.552960 & 0.742216 \\
\hline $\mathrm{H}$ & -2.414024 & 3.285519 & 1.450670 \\
\hline $\mathrm{H}$ & -2.613787 & 2.933588 & -0.267423 \\
\hline $\mathrm{C}$ & -4.316301 & 2.387789 & 0.921100 \\
\hline $\mathrm{H}$ & -4.839702 & 3.304833 & 0.637978 \\
\hline $\mathrm{H}$ & -4.556091 & 2.176041 & 1.969658 \\
\hline $\mathrm{C}$ & -4.807406 & 1.242100 & 0.051036 \\
\hline $\mathrm{H}$ & -4.669365 & 1.480348 & -1.011728 \\
\hline
\end{tabular}




$\begin{array}{lrrr}\text { H } & -5.873441 & 1.066489 & 0.218499 \\ \mathrm{C} & -4.807939 & -1.241838 & 0.049630 \\ \mathrm{H} & -5.873849 & -1.066145 & 0.217921 \\ \mathrm{H} & -4.670553 & -1.478612 & -1.013555 \\ \mathrm{C} & -4.316807 & -2.388971 & 0.917770 \\ \mathrm{H} & -4.840571 & -3.305430 & 0.633408 \\ \mathrm{H} & -4.556189 & -2.178775 & 1.966738 \\ \mathrm{C} & -2.812249 & -2.554251 & 0.738157 \\ \mathrm{H} & -2.414611 & -3.288056 & 1.445288 \\ \mathrm{H} & -2.614766 & -2.933183 & -0.272179 \\ \mathrm{H} & -0.276418 & -2.158749 & 1.495465 \\ \mathrm{H} & -0.275996 & 2.155754 & 1.498918\end{array}$

Julolidine $\pi$-dimer singlet $\mathrm{E}(\mathrm{UB} 98)=-1487.98421024$ a.u.; triplet $\mathrm{E}(\mathrm{U} \omega \mathrm{B} 97 \mathrm{XD})=$ 1487.97792452 a.u.

\begin{tabular}{lrrr} 
Atomic & \multicolumn{3}{c}{ Coordinates(Angstroms) } \\
type & $\mathrm{X}$ & $\mathrm{Y}$ & $\mathrm{Z}$ \\
$\mathrm{N}$ & 4.223992 & 0.000014 & -0.345623 \\
$\mathrm{~N}$ & -2.324677 & -2.218308 & -2.746523 \\
$\mathrm{~N}$ & -2.324903 & 2.217835 & -2.746733 \\
$\mathrm{C}$ & 2.908385 & -0.000027 & -0.707015 \\
$\mathrm{C}$ & 2.212027 & -1.243002 & -0.938642 \\
$\mathrm{C}$ & 0.910085 & -1.218043 & -1.391131 \\
$\mathrm{C}$ & 0.208253 & -0.000118 & -1.642019 \\
$\mathrm{C}$ & 0.910047 & 1.217851 & -1.391245 \\
$\mathrm{C}$ & 2.211986 & 1.242902 & -0.938764 \\
$\mathrm{C}$ & -1.121595 & -0.000148 & -2.157678 \\
$\mathrm{C}$ & -1.791133 & -1.210007 & -2.484786 \\
$\mathrm{C}$ & -1.791123 & 1.209697 & -2.484844 \\
$\mathrm{C}$ & 2.915491 & -2.560900 & -0.690239 \\
$\mathrm{H}$ & 2.515770 & -3.324182 & -1.367575 \\
$\mathrm{H}$ & 2.704028 & -2.894442 & 0.336681 \\
$\mathrm{C}$ & 4.431415 & -2.414354 & -0.862587 \\
$\mathrm{H}$ & 4.946633 & -3.329058 & -0.549091 \\
$\mathrm{H}$ & 4.683110 & -2.234153 & -1.916173 \\
$\mathrm{C}$ & 4.929046 & -1.245628 & -0.012522 \\
$\mathrm{H}$ & 4.799867 & -1.468472 & 1.057458 \\
$\mathrm{H}$ & 5.996157 & -1.069645 & -0.187793 \\
$\mathrm{C}$ & 4.928994 & 1.245713 & -0.012622
\end{tabular}




\begin{tabular}{|c|c|c|c|}
\hline $\mathrm{H}$ & 5.996115 & 1.069750 & -0.187856 \\
\hline $\mathrm{H}$ & 4.799785 & 1.468650 & 1.057334 \\
\hline $\mathrm{C}$ & 4.431341 & 2.414341 & -0.862808 \\
\hline $\mathrm{H}$ & 4.946520 & 3.329093 & -0.549387 \\
\hline $\mathrm{H}$ & 4.683073 & 2.234048 & -1.916370 \\
\hline $\mathrm{C}$ & 2.915406 & 2.560855 & -0.690524 \\
\hline $\mathrm{H}$ & 2.515684 & 3.324021 & -1.367991 \\
\hline $\mathrm{H}$ & 2.703886 & 2.894559 & 0.336331 \\
\hline $\mathrm{H}$ & 0.407096 & 2.161955 & -1.587527 \\
\hline $\mathrm{H}$ & 0.407165 & -2.162183 & -1.587314 \\
\hline $\mathrm{N}$ & -4.223987 & 0.000023 & 0.345570 \\
\hline $\mathrm{N}$ & 2.324802 & 2.218191 & 2.746547 \\
\hline $\mathrm{N}$ & 2.324976 & -2.217834 & 2.746777 \\
\hline $\mathrm{C}$ & -2.908395 & 0.000044 & 0.707013 \\
\hline $\mathrm{C}$ & -2.212025 & 1.243009 & 0.938660 \\
\hline $\mathrm{C}$ & -0.910092 & 1.218027 & 1.391173 \\
\hline $\mathrm{C}$ & -0.208285 & 0.000091 & 1.642080 \\
\hline $\mathrm{C}$ & -0.910093 & -1.217867 & 1.391295 \\
\hline $\mathrm{C}$ & -2.212022 & -1.242897 & 0.938781 \\
\hline $\mathrm{C}$ & 1.121559 & 0.000102 & 2.157751 \\
\hline $\mathrm{C}$ & 1.791145 & 1.209957 & 2.484776 \\
\hline $\mathrm{C}$ & 1.791094 & -1.209752 & 2.484881 \\
\hline $\mathrm{C}$ & -2.915468 & 2.560918 & 0.690253 \\
\hline $\mathrm{H}$ & -2.515763 & 3.324187 & 1.367615 \\
\hline $\mathrm{H}$ & -2.703965 & 2.894476 & -0.336653 \\
\hline $\mathrm{C}$ & -4.431398 & 2.414390 & 0.862546 \\
\hline $\mathrm{H}$ & -4.946593 & 3.329103 & 0.549038 \\
\hline $\mathrm{H}$ & -4.683133 & 2.234187 & 1.916123 \\
\hline $\mathrm{C}$ & -4.929017 & 1.245676 & 0.012457 \\
\hline $\mathrm{H}$ & -4.799806 & 1.468525 & -1.057518 \\
\hline $\mathrm{H}$ & -5.996135 & 1.069703 & 0.187698 \\
\hline $\mathrm{C}$ & -4.928993 & -1.245664 & 0.012536 \\
\hline $\mathrm{H}$ & -5.996120 & -1.069685 & 0.187722 \\
\hline $\mathrm{H}$ & -4.799741 & -1.468603 & -1.057415 \\
\hline $\mathrm{C}$ & -4.431397 & -2.414300 & 0.862744 \\
\hline $\mathrm{H}$ & -4.946575 & -3.329045 & 0.549301 \\
\hline $\mathrm{H}$ & -4.683174 & -2.234002 & 1.916294 \\
\hline $\mathrm{C}$ & -2.915456 & -2.560840 & 0.690530 \\
\hline $\mathrm{H}$ & -2.515779 & -3.324001 & 1.368028 \\
\hline $\mathrm{H}$ & -2.703890 & -2.894567 & -0.336309 \\
\hline
\end{tabular}




$\begin{array}{llrr}\mathrm{H} & -0.407163 & -2.161979 & 1.587589 \\ \mathrm{H} & -0.407160 & 2.162159 & 1.587367\end{array}$

Julolidine $\pi$-dimer singlet $E(U B 97 D)=-1487.54190987$ a.u.

\begin{tabular}{|c|c|c|c|}
\hline \multirow{2}{*}{$\begin{array}{c}\text { Atomic } \\
\text { type }\end{array}$} & \multicolumn{3}{|c|}{ Coordinates(Angstroms) } \\
\hline & $\mathrm{X}$ & $\mathrm{Y}$ & Z \\
\hline $\mathrm{N}$ & -4.13584 & 0.00006 & -0.39419 \\
\hline $\mathrm{N}$ & 2.51446 & 2.21314 & -2.60034 \\
\hline $\mathrm{N}$ & 2.51481 & -2.21358 & -2.60014 \\
\hline $\mathrm{C}$ & -2.80647 & -0.00002 & -0.72040 \\
\hline $\mathrm{C}$ & -2.10547 & 1.24529 & -0.93808 \\
\hline $\mathrm{C}$ & -0.78047 & 1.21983 & -1.33220 \\
\hline $\mathrm{C}$ & -0.06884 & -0.00020 & -1.55082 \\
\hline $\mathrm{C}$ & -0.78061 & -1.22013 & -1.33213 \\
\hline $\mathrm{C}$ & -2.10561 & -1.24543 & -0.93800 \\
\hline $\mathrm{C}$ & 1.28833 & -0.00029 & -2.00513 \\
\hline $\mathrm{C}$ & 1.96773 & 1.20494 & -2.32604 \\
\hline $\mathrm{C}$ & 1.96755 & -1.20559 & -2.32610 \\
\hline $\mathrm{C}$ & -2.81974 & 2.56727 & -0.74666 \\
\hline $\mathrm{H}$ & -2.41085 & 3.30894 & -1.44862 \\
\hline $\mathrm{H}$ & -2.62910 & 2.93698 & 0.27380 \\
\hline $\mathrm{C}$ & -4.33694 & 2.41052 & -0.93395 \\
\hline $\mathrm{H}$ & -4.86028 & 3.33214 & -0.64272 \\
\hline $\mathrm{H}$ & -4.57393 & 2.19881 & -1.98882 \\
\hline $\mathrm{C}$ & -4.83882 & 1.25390 & -0.06298 \\
\hline $\mathrm{H}$ & -4.69048 & 1.48734 & 1.00451 \\
\hline $\mathrm{H}$ & -5.91086 & 1.07815 & -0.23162 \\
\hline $\mathrm{C}$ & -4.83896 & -1.25369 & -0.06289 \\
\hline $\mathrm{H}$ & -5.91098 & -1.07784 & -0.23156 \\
\hline $\mathrm{H}$ & -4.69066 & -1.48707 & 1.00462 \\
\hline $\mathrm{C}$ & -4.33720 & -2.41043 & -0.93377 \\
\hline $\mathrm{H}$ & -4.86063 & -3.33197 & -0.64245 \\
\hline $\mathrm{H}$ & -4.57418 & -2.19878 & -1.98866 \\
\hline $\mathrm{C}$ & -2.82001 & -2.56732 & -0.74650 \\
\hline $\mathrm{H}$ & -2.41122 & -3.30904 & -1.44847 \\
\hline $\mathrm{H}$ & -2.62937 & -2.93705 & 0.27394 \\
\hline $\mathrm{H}$ & -0.27039 & -2.16766 & -1.50809 \\
\hline $\mathrm{H}$ & -0.27014 & 2.16729 & -1.50821 \\
\hline $\mathrm{N}$ & 4.13587 & 0.00009 & 0.39437 \\
\hline $\mathrm{N}$ & -2.51475 & -2.21324 & 2.60031 \\
\hline
\end{tabular}




$\begin{array}{lrrr}\mathrm{N} & -2.51418 & 2.21363 & 2.60017 \\ \mathrm{C} & 2.80645 & 0.00007 & 0.72037 \\ \mathrm{C} & 2.10554 & -1.24531 & 0.93802 \\ \mathrm{C} & 0.78049 & -1.21994 & 1.33200 \\ \mathrm{C} & 0.06871 & 0.00003 & 1.55047 \\ \mathrm{C} & 0.78039 & 1.22002 & 1.33180 \\ \mathrm{C} & 2.10545 & 1.24542 & 0.93783 \\ \mathrm{C} & -1.28844 & 0.00002 & 2.00481 \\ \mathrm{C} & -1.96754 & -1.20523 & 2.32628 \\ \mathrm{C} & -1.96763 & 1.20529 & 2.32602 \\ \mathrm{C} & 2.81995 & -2.56723 & 0.74673 \\ \mathrm{H} & 2.41101 & -3.30891 & 1.44866 \\ \mathrm{H} & 2.62948 & -2.93699 & -0.27374 \\ \mathrm{C} & 4.33710 & -2.41035 & 0.93425 \\ \mathrm{H} & 4.86057 & -3.33193 & 0.64312 \\ \mathrm{H} & 4.57391 & -2.19859 & 1.98915 \\ \mathrm{C} & 4.83902 & -1.25371 & 0.06333 \\ \mathrm{H} & 4.69090 & -1.48720 & -1.00418 \\ \mathrm{H} & 5.91102 & -1.07785 & 0.23215 \\ \mathrm{C} & 4.83890 & 1.25388 & 0.06306 \\ \mathrm{H} & 5.91092 & 1.07814 & 0.23183 \\ \mathrm{H} & 4.69067 & 1.48719 & -1.00448 \\ \mathrm{C} & 4.33695 & 2.41062 & 0.93384 \\ \mathrm{H} & 4.86033 & 3.33220 & 0.64254 \\ \mathrm{H} & 2.87383 & 2.19904 & 1.98875 \\ \mathrm{C} & 0.27008 & 2.16751 & 1.50768 \\ \mathrm{H} & 2.41083 & 3.30909 & 1.44828 \\ \mathrm{H} & -2.16744 & 1.50804\end{array}$

1 radical $\mathrm{E}(\mathrm{U} \omega \mathrm{B} 97 \mathrm{XD})=-1054.85182646$ a.u.

\begin{tabular}{lccc} 
Atomic & \multicolumn{3}{c}{ Coordinates(Angstroms) } \\
\multicolumn{1}{c}{ type } & $\mathrm{X}$ & $\mathrm{Y}$ & $\mathrm{Z}$ \\
$\mathrm{N}$ & -3.224609 & -0.377365 & -0.094830 \\
$\mathrm{C}$ & -1.877714 & -0.088199 & 0.003813 \\
$\mathrm{C}$ & -3.894579 & 1.913163 & 0.374246 \\
$\mathrm{H}$ & -3.979731 & 1.673123 & 1.440821 \\
$\mathrm{H}$ & -4.642563 & 2.681322 & 0.149818 \\
$\mathrm{C}$ & -4.183272 & 0.661571 & -0.423201
\end{tabular}




\begin{tabular}{|c|c|c|c|}
\hline $\mathrm{H}$ & -5.178384 & 0.280533 & -0.175949 \\
\hline $\mathrm{H}$ & -4.180345 & 0.871029 & -1.505436 \\
\hline $\mathrm{C}$ & -3.631172 & -1.724031 & -0.431670 \\
\hline $\mathrm{H}$ & -3.483167 & -1.921929 & -1.505987 \\
\hline $\mathrm{H}$ & -4.702465 & -1.816059 & -0.231681 \\
\hline $\mathrm{C}$ & -1.482230 & 1.300497 & 0.075820 \\
\hline $\mathrm{C}$ & -0.152339 & 1.598704 & 0.108662 \\
\hline $\mathrm{C}$ & 2.203535 & 0.994023 & 0.041610 \\
\hline $\mathrm{C}$ & 3.236820 & 0.053548 & -0.031550 \\
\hline $\mathrm{C}$ & 4.604845 & 0.451161 & -0.047012 \\
\hline $\mathrm{C}$ & 0.855850 & 0.608456 & 0.055695 \\
\hline $\mathrm{C}$ & 0.470477 & -0.767421 & 0.020163 \\
\hline $\mathrm{C}$ & 1.558109 & -1.706454 & -0.076775 \\
\hline $\mathrm{H}$ & 1.359827 & -2.762773 & -0.158042 \\
\hline $\mathrm{C}$ & 2.864498 & -1.326147 & -0.103096 \\
\hline $\mathrm{H}$ & 3.639795 & -2.081146 & -0.187270 \\
\hline $\mathrm{C}$ & -0.906039 & -1.123051 & 0.053047 \\
\hline $\mathrm{C}$ & -2.852703 & -2.692704 & 0.423377 \\
\hline $\mathrm{H}$ & -3.185095 & -3.719610 & 0.237504 \\
\hline $\mathrm{H}$ & -3.066809 & -2.464151 & 1.473879 \\
\hline $\mathrm{C}$ & -2.472160 & 3.140776 & -1.305113 \\
\hline $\mathrm{H}$ & -1.482816 & 3.558518 & -1.513538 \\
\hline $\mathrm{H}$ & -3.200968 & 3.958799 & -1.333360 \\
\hline $\mathrm{H}$ & -2.707683 & 2.441271 & -2.112434 \\
\hline $\mathrm{C}$ & -2.154051 & 3.497507 & 1.154891 \\
\hline $\mathrm{H}$ & -2.060853 & 3.027980 & 2.138844 \\
\hline $\mathrm{H}$ & -2.952551 & 4.245727 & 1.204027 \\
\hline $\mathrm{H}$ & -1.223852 & 4.031653 & 0.943639 \\
\hline $\mathrm{C}$ & -2.492502 & 2.454453 & 0.075600 \\
\hline $\mathrm{C}$ & -1.337560 & -2.603042 & 0.166876 \\
\hline $\mathrm{C}$ & -1.039390 & -3.387463 & -1.129953 \\
\hline $\mathrm{H}$ & -1.253581 & -4.451305 & -0.976901 \\
\hline $\mathrm{H}$ & -0.005036 & -3.296772 & -1.462054 \\
\hline $\mathrm{H}$ & -1.664397 & -3.031259 & -1.954089 \\
\hline $\mathrm{C}$ & -0.693976 & -3.294231 & 1.391417 \\
\hline $\mathrm{H}$ & -0.835694 & -2.683572 & 2.288346 \\
\hline $\mathrm{H}$ & 0.372220 & -3.490695 & 1.292124 \\
\hline $\mathrm{H}$ & -1.186857 & -4.258753 & 1.557121 \\
\hline $\mathrm{H}$ & 0.166698 & 2.635526 & 0.156548 \\
\hline $\mathrm{H}$ & 2.441638 & 2.053582 & 0.086128 \\
\hline
\end{tabular}




$\begin{array}{lrrr}\mathrm{C} & 5.650518 & -0.503308 & -0.127933 \\ \mathrm{~N} & 6.487650 & -1.308820 & -0.195533 \\ \mathrm{C} & 4.973678 & 1.819647 & 0.015454 \\ \mathrm{~N} & 5.239933 & 2.951074 & 0.067753\end{array}$

$1 \sigma$-dimer $\mathrm{E}(\mathrm{RB} 98)=-2109.51805928$ a.u.

\begin{tabular}{|c|c|c|c|}
\hline \multirow{2}{*}{$\begin{array}{l}\text { Atomic } \\
\text { type }\end{array}$} & \multicolumn{3}{|c|}{ Coordinates(Angstroms) } \\
\hline & $\mathrm{X}$ & $\mathrm{Y}$ & Z \\
\hline $\mathrm{N}$ & 0.419137 & -2.985806 & 2.059041 \\
\hline $\mathrm{N}$ & 0.437523 & 1.304300 & 2.036282 \\
\hline $\mathrm{N}$ & -8.167834 & 0.559750 & -0.385413 \\
\hline $\mathrm{N}$ & -0.419156 & -2.985769 & -2.059069 \\
\hline $\mathrm{N}$ & -0.437503 & 1.304333 & -2.036247 \\
\hline $\mathrm{C}$ & -0.381389 & -0.844018 & 0.737363 \\
\hline $\mathrm{C}$ & 0.082362 & -2.036568 & 1.481973 \\
\hline $\mathrm{C}$ & 0.090313 & 0.351328 & 1.471615 \\
\hline $\mathrm{C}$ & -1.915078 & -0.841842 & 0.616248 \\
\hline $\mathrm{C}$ & -2.632348 & -2.061484 & 0.505758 \\
\hline $\mathrm{H}$ & -2.105407 & -3.010986 & 0.525325 \\
\hline $\mathrm{C}$ & -4.004537 & -2.063113 & 0.366337 \\
\hline $\mathrm{H}$ & -4.482866 & -3.025258 & 0.259831 \\
\hline $\mathrm{C}$ & -4.780532 & -0.859529 & 0.321710 \\
\hline $\mathrm{C}$ & -6.211148 & -0.814456 & 0.173816 \\
\hline $\mathrm{C}$ & -7.080607 & -2.093472 & 0.304618 \\
\hline $\mathrm{C}$ & -6.963499 & -3.012115 & -0.941948 \\
\hline $\mathrm{H}$ & -7.571374 & -3.915904 & -0.800818 \\
\hline $\mathrm{H}$ & -5.934819 & -3.326371 & -1.136601 \\
\hline $\mathrm{H}$ & -7.315518 & -2.502122 & -1.845413 \\
\hline $\mathrm{C}$ & -6.758433 & -2.896027 & 1.598809 \\
\hline $\mathrm{H}$ & -7.546799 & -3.642493 & 1.761543 \\
\hline $\mathrm{H}$ & -6.740100 & -2.227854 & 2.467543 \\
\hline $\mathrm{H}$ & -5.808985 & -3.431774 & 1.573558 \\
\hline $\mathrm{C}$ & -8.564245 & -1.679405 & 0.474364 \\
\hline $\mathrm{H}$ & -9.199226 & -2.568977 & 0.376442 \\
\hline $\mathrm{H}$ & -8.716412 & -1.279229 & 1.486058 \\
\hline $\mathrm{C}$ & -8.995877 & -0.624641 & -0.525583 \\
\hline $\mathrm{H}$ & -8.953712 & -1.008739 & -1.560747 \\
\hline $\mathrm{H}$ & -10.037892 & -0.330770 & -0.339877 \\
\hline $\mathrm{C}$ & -6.821534 & 0.443574 & -0.067707 \\
\hline $\mathrm{C}$ & -8.763168 & 1.747766 & -0.999791 \\
\hline
\end{tabular}




\begin{tabular}{|c|c|c|c|}
\hline $\mathrm{H}$ & -9.591798 & 2.091423 & -0.360784 \\
\hline $\mathrm{H}$ & -9.212180 & 1.454281 & -1.962334 \\
\hline $\mathrm{C}$ & -7.767073 & 2.878786 & -1.228830 \\
\hline $\mathrm{H}$ & -8.320736 & 3.813824 & -1.384549 \\
\hline $\mathrm{H}$ & -7.183110 & 2.689851 & -2.139205 \\
\hline $\mathrm{C}$ & -6.789954 & 3.016763 & -0.040623 \\
\hline $\mathrm{C}$ & -7.556874 & 3.298555 & 1.279881 \\
\hline $\mathrm{H}$ & -6.846884 & 3.452455 & 2.100313 \\
\hline $\mathrm{H}$ & -8.213088 & 2.468345 & 1.560400 \\
\hline $\mathrm{H}$ & -8.168100 & 4.205683 & 1.181968 \\
\hline $\mathrm{C}$ & -5.845365 & 4.202844 & -0.321357 \\
\hline $\mathrm{H}$ & -5.163796 & 4.395587 & 0.514742 \\
\hline $\mathrm{H}$ & -6.440078 & 5.112603 & -0.469470 \\
\hline $\mathrm{H}$ & -5.246070 & 4.037710 & -1.224450 \\
\hline $\mathrm{C}$ & -6.059003 & 1.674103 & 0.064265 \\
\hline $\mathrm{C}$ & -4.710270 & 1.601858 & 0.296243 \\
\hline $\mathrm{H}$ & -4.124579 & 2.510441 & 0.396184 \\
\hline $\mathrm{C}$ & -4.026952 & 0.361368 & 0.393210 \\
\hline $\mathrm{C}$ & -2.620552 & 0.347545 & 0.558479 \\
\hline $\mathrm{H}$ & -2.107541 & 1.303349 & 0.632558 \\
\hline $\mathrm{C}$ & 0.381386 & -0.844009 & -0.737355 \\
\hline $\mathrm{C}$ & -0.082366 & -2.036550 & -1.481978 \\
\hline $\mathrm{C}$ & -0.090315 & 0.351346 & -1.471593 \\
\hline $\mathrm{C}$ & 1.915075 & -0.841835 & -0.616240 \\
\hline $\mathrm{C}$ & 2.620550 & 0.347551 & -0.558461 \\
\hline $\mathrm{H}$ & 2.107539 & 1.303355 & -0.632533 \\
\hline $\mathrm{C}$ & 4.026950 & 0.361371 & -0.393195 \\
\hline $\mathrm{C}$ & 4.710270 & 1.601860 & -0.296225 \\
\hline $\mathrm{H}$ & 4.124580 & 2.510444 & -0.396160 \\
\hline $\mathrm{C}$ & 6.059005 & 1.674102 & -0.064256 \\
\hline $\mathrm{C}$ & 6.789961 & 3.016761 & 0.040626 \\
\hline $\mathrm{C}$ & 6.821535 & 0.443572 & 0.067706 \\
\hline $\mathrm{C}$ & 8.995886 & -0.624647 & 0.525544 \\
\hline $\mathrm{H}$ & 8.953739 & -1.008747 & 1.560708 \\
\hline $\mathrm{H}$ & 10.037897 & -0.330777 & 0.339819 \\
\hline $\mathrm{C}$ & 8.564234 & -1.679409 & -0.474396 \\
\hline $\mathrm{H}$ & 9.199216 & -2.568982 & -0.376489 \\
\hline $\mathrm{H}$ & 8.716379 & -1.279232 & -1.486093 \\
\hline $\mathrm{C}$ & 7.080599 & -2.093475 & -0.304616 \\
\hline $\mathrm{C}$ & 6.758393 & -2.896045 & -1.598790 \\
\hline
\end{tabular}




$\begin{array}{lrrr}\mathrm{H} & 5.808939 & -3.431780 & -1.573512 \\ \mathrm{H} & 7.546747 & -3.642521 & -1.761529 \\ \mathrm{H} & 6.740050 & -2.227884 & -2.467533 \\ \mathrm{C} & 6.963519 & -3.012102 & 0.941963 \\ \mathrm{H} & 5.934843 & -3.326355 & 1.136645 \\ \mathrm{H} & 7.315561 & -2.502099 & 1.845413 \\ \mathrm{H} & 7.571389 & -3.915894 & 0.800830 \\ \mathrm{C} & 6.211145 & -0.814457 & -0.173811 \\ \mathrm{C} & 4.780529 & -0.859527 & -0.321704 \\ \mathrm{C} & 4.004534 & -2.063110 & -0.366346 \\ \mathrm{H} & 4.482863 & -3.025257 & -0.259855 \\ \mathrm{C} & 2.632344 & -2.061480 & -0.505765 \\ \mathrm{H} & 2.105403 & -3.010980 & -0.525343 \\ \mathrm{~N} & 8.167840 & 0.559745 & 0.385393 \\ \mathrm{C} & 7.556866 & 3.298553 & -1.279887 \\ \mathrm{H} & 8.168094 & 4.205680 & -1.181979 \\ \mathrm{H} & 6.846867 & 3.452456 & -2.100311 \\ \mathrm{H} & 8.213076 & 2.468342 & -1.560414 \\ \mathrm{C} & 5.845377 & 4.202843 & 0.321373 \\ \mathrm{H} & 6.440094 & 5.112600 & 0.469480 \\ \mathrm{H} & 5.246093 & 4.037709 & 1.224472 \\ \mathrm{H} & 5.163799 & 4.395588 & -0.514718 \\ \mathrm{C} & 7.767093 & 2.878779 & 1.228821 \\ \mathrm{H} & 8.320759 & 3.813816 & 1.384536 \\ \mathrm{C} & 8.763184 & 1.747758 & 0.999768 \\ \mathrm{H} & 9.591807 & 2.091415 & 0.360751 \\ \mathrm{H} & 9.212207 & 1.454269 & 1.962304 \\ \mathrm{H} & 7.183141 & 2.689843 & 2.139203\end{array}$

$1 \sigma$-dimer $\mathrm{E}(\mathrm{RB} 97 \mathrm{D})=-2108.86877878$ a.u.

\begin{tabular}{lrrr} 
Atomic & \multicolumn{3}{c}{ Coordinates(Angstroms) } \\
type & $\mathrm{X}$ & $\mathrm{Y}$ & $\mathrm{Z}$ \\
$\mathrm{N}$ & 0.521081 & -3.038613 & 2.016846 \\
$\mathrm{~N}$ & 0.549174 & 1.285832 & 2.006593 \\
$\mathrm{~N}$ & -8.124252 & 0.568999 & -0.411193 \\
$\mathrm{~N}$ & -0.521400 & -3.037998 & -2.017354 \\
$\mathrm{~N}$ & -0.549002 & 1.286451 & -2.005838 \\
$\mathrm{C}$ & -0.358579 & -0.873403 & 0.774821 \\
$\mathrm{C}$ & 0.146284 & -2.069877 & 1.479070 \\
$\mathrm{C}$ & 0.160592 & 0.318428 & 1.476144
\end{tabular}




\begin{tabular}{|c|c|c|c|}
\hline $\mathrm{C}$ & -1.887745 & -0.864245 & 0.689428 \\
\hline $\mathrm{C}$ & -2.612595 & -2.082964 & 0.582362 \\
\hline $\mathrm{H}$ & -2.085198 & -3.035657 & 0.603778 \\
\hline $\mathrm{C}$ & -3.989453 & -2.076426 & 0.433666 \\
\hline $\mathrm{H}$ & -4.474021 & -3.038481 & 0.323194 \\
\hline $\mathrm{C}$ & -4.755762 & -0.864497 & 0.372329 \\
\hline $\mathrm{C}$ & -6.183750 & -0.807056 & 0.218828 \\
\hline $\mathrm{C}$ & -7.070881 & -2.065879 & 0.382356 \\
\hline $\mathrm{C}$ & -6.967014 & -3.009831 & -0.845350 \\
\hline $\mathrm{H}$ & -7.590227 & -3.904208 & -0.679722 \\
\hline $\mathrm{H}$ & -5.937877 & -3.336097 & -1.036880 \\
\hline $\mathrm{H}$ & -7.320888 & -2.503583 & -1.755195 \\
\hline $\mathrm{C}$ & -6.762963 & -2.840953 & 1.696799 \\
\hline $\mathrm{H}$ & -7.552671 & -3.592738 & 1.857043 \\
\hline $\mathrm{H}$ & -6.769191 & -2.145732 & 2.549486 \\
\hline $\mathrm{H}$ & -5.804387 & -3.367629 & 1.700341 \\
\hline $\mathrm{C}$ & -8.551577 & -1.629781 & 0.538528 \\
\hline $\mathrm{H}$ & -9.194762 & -2.520821 & 0.479450 \\
\hline $\mathrm{H}$ & -8.693874 & -1.176184 & 1.532705 \\
\hline $\mathrm{C}$ & -8.968826 & -0.613429 & -0.509940 \\
\hline $\mathrm{H}$ & -8.913888 & -1.042460 & -1.530444 \\
\hline $\mathrm{H}$ & -10.012136 & -0.298055 & -0.345528 \\
\hline $\mathrm{C}$ & -6.781434 & 0.454165 & -0.070230 \\
\hline $\mathrm{C}$ & -8.707499 & 1.748262 & -1.064213 \\
\hline $\mathrm{H}$ & -9.533613 & 2.118171 & -0.430429 \\
\hline $\mathrm{H}$ & -9.155276 & 1.429620 & -2.023592 \\
\hline $\mathrm{C}$ & -7.695581 & 2.864920 & -1.313937 \\
\hline $\mathrm{H}$ & -8.239139 & 3.805608 & -1.492760 \\
\hline $\mathrm{H}$ & -7.098866 & 2.643980 & -2.212710 \\
\hline $\mathrm{C}$ & -6.732550 & 3.021605 & -0.114679 \\
\hline $\mathrm{C}$ & -7.524059 & 3.319795 & 1.187638 \\
\hline $\mathrm{H}$ & -6.822604 & 3.484439 & 2.018476 \\
\hline $\mathrm{H}$ & -8.183657 & 2.483428 & 1.457293 \\
\hline $\mathrm{H}$ & -8.136658 & 4.227338 & 1.059406 \\
\hline $\mathrm{C}$ & -5.787342 & 4.204897 & -0.400587 \\
\hline $\mathrm{H}$ & -5.117350 & 4.404061 & 0.448644 \\
\hline $\mathrm{H}$ & -6.388011 & 5.111307 & -0.571904 \\
\hline $\mathrm{H}$ & -5.173458 & 4.016058 & -1.294020 \\
\hline $\mathrm{C}$ & -6.008613 & 1.682249 & 0.033083 \\
\hline $\mathrm{C}$ & -4.658685 & 1.605698 & 0.288061 \\
\hline
\end{tabular}




\begin{tabular}{|c|c|c|c|}
\hline $\mathrm{H}$ & -4.063585 & 2.513878 & 0.371767 \\
\hline $\mathrm{C}$ & -3.988295 & 0.360045 & 0.424781 \\
\hline $\mathrm{C}$ & -2.583148 & 0.337294 & 0.602351 \\
\hline $\mathrm{H}$ & -2.056922 & 1.290850 & 0.652755 \\
\hline $\mathrm{C}$ & 0.358526 & -0.873242 & -0.774716 \\
\hline $\mathrm{C}$ & -0.146354 & -2.069560 & -1.479216 \\
\hline $\mathrm{C}$ & -0.160633 & 0.318744 & -1.475786 \\
\hline $\mathrm{C}$ & 1.887692 & -0.864122 & -0.689327 \\
\hline $\mathrm{C}$ & 2.583114 & 0.337393 & -0.602062 \\
\hline $\mathrm{H}$ & 2.056903 & 1.290965 & -0.652319 \\
\hline $\mathrm{C}$ & 3.988267 & 0.360096 & -0.424529 \\
\hline $\mathrm{C}$ & 4.658695 & 1.605722 & -0.287741 \\
\hline $\mathrm{H}$ & 4.063611 & 2.513922 & -0.371348 \\
\hline $\mathrm{C}$ & 6.008660 & 1.682227 & -0.032941 \\
\hline $\mathrm{C}$ & 6.732653 & 3.021565 & 0.114736 \\
\hline $\mathrm{C}$ & 6.781468 & 0.454120 & 0.070206 \\
\hline $\mathrm{C}$ & 8.969004 & -0.613507 & 0.509205 \\
\hline $\mathrm{H}$ & 8.914431 & -1.042584 & 1.529710 \\
\hline $\mathrm{H}$ & 10.012253 & -0.298119 & 0.344431 \\
\hline $\mathrm{C}$ & 8.551371 & -1.629832 & -0.539141 \\
\hline $\mathrm{H}$ & 9.194592 & -2.520863 & -0.480335 \\
\hline $\mathrm{H}$ & 8.693242 & -1.176217 & -1.533371 \\
\hline $\mathrm{C}$ & 7.070757 & -2.065939 & -0.382291 \\
\hline $\mathrm{C}$ & 6.762226 & -2.841386 & -1.696368 \\
\hline $\mathrm{H}$ & 5.803557 & -3.367908 & -1.699349 \\
\hline $\mathrm{H}$ & 7.551754 & -3.593345 & -1.856680 \\
\hline $\mathrm{H}$ & 6.768220 & -2.146438 & -2.549279 \\
\hline $\mathrm{C}$ & 6.967473 & -3.009526 & 0.845746 \\
\hline $\mathrm{H}$ & 5.938434 & -3.335717 & 1.037910 \\
\hline $\mathrm{H}$ & 7.321825 & -2.503009 & 1.755257 \\
\hline $\mathrm{H}$ & 7.590586 & -3.903964 & 0.680073 \\
\hline $\mathrm{C}$ & 6.183697 & -0.807081 & -0.218751 \\
\hline $\mathrm{C}$ & 4.755708 & -0.864469 & -0.372245 \\
\hline $\mathrm{C}$ & 3.989392 & -2.076378 & -0.433852 \\
\hline $\mathrm{H}$ & 4.473959 & -3.038466 & -0.323661 \\
\hline $\mathrm{C}$ & 2.612531 & -2.082871 & -0.582530 \\
\hline $\mathrm{H}$ & 2.085126 & -3.035555 & -0.604157 \\
\hline $\mathrm{N}$ & 8.124380 & 0.568920 & 0.410815 \\
\hline $\mathrm{C}$ & 7.523863 & 3.319818 & -1.187748 \\
\hline $\mathrm{H}$ & 8.136501 & 4.227348 & -1.059608 \\
\hline
\end{tabular}




$\begin{array}{lrrr}\mathrm{H} & 6.822217 & 3.484516 & -2.018415 \\ \mathrm{H} & 8.183390 & 2.483460 & -1.457603 \\ \mathrm{C} & 5.787529 & 4.204855 & 0.400928 \\ \mathrm{H} & 6.388250 & 5.111248 & 0.572150 \\ \mathrm{H} & 5.173852 & 4.015977 & 1.294496 \\ \mathrm{H} & 5.117339 & 4.404071 & -0.448135 \\ \mathrm{C} & 7.695968 & 2.864803 & 1.313756 \\ \mathrm{H} & 8.239578 & 3.805477 & 1.492498 \\ \mathrm{C} & 8.707817 & 1.748153 & 1.063719 \\ \mathrm{H} & 9.533760 & 2.118083 & 0.429725 \\ \mathrm{H} & 9.155851 & 1.429471 & 2.022964 \\ \mathrm{H} & 7.099470 & 2.643818 & 2.212663\end{array}$

$1 \sigma$-dimer $\mathrm{E}(\mathrm{U} \omega \mathrm{B} 97 \mathrm{XD})=-2109.74169098$ a.u. Atomic Coordinates(Angstroms)

$\begin{array}{lrrr}\text { type } & \mathrm{X} & \mathrm{Y} & \mathrm{Z} \\ \mathrm{N} & 0.438745 & -3.012552 & 2.023570 \\ \mathrm{~N} & 0.464845 & 1.275724 & 2.012639 \\ \mathrm{~N} & -8.106842 & 0.571663 & -0.360188 \\ \mathrm{~N} & -0.438756 & -3.012546 & -2.023568 \\ \mathrm{~N} & -0.464841 & 1.275729 & -2.012620 \\ \mathrm{C} & -0.362171 & -0.866647 & 0.727669 \\ \mathrm{C} & 0.099951 & -2.059452 & 1.463913 \\ \mathrm{C} & 0.113462 & 0.323104 & 1.459851 \\ \mathrm{C} & -1.889946 & -0.857529 & 0.618348 \\ \mathrm{C} & -2.609483 & -2.068435 & 0.509281 \\ \mathrm{H} & -2.088085 & -3.019979 & 0.527749 \\ \mathrm{C} & -3.973151 & -2.059800 & 0.368452 \\ \mathrm{H} & -4.457763 & -3.016763 & 0.257578 \\ \mathrm{C} & -4.734811 & -0.853549 & 0.324381 \\ \mathrm{C} & -6.161802 & -0.798533 & 0.188614 \\ \mathrm{C} & -7.032468 & -2.060354 & 0.354998 \\ \mathrm{C} & -6.953818 & -2.983533 & -0.877988 \\ \mathrm{H} & -7.552591 & -3.886704 & -0.711063 \\ \mathrm{H} & -5.931795 & -3.295308 & -1.101245 \\ \mathrm{H} & -7.333110 & -2.482042 & -1.772927 \\ \mathrm{C} & -6.684503 & -2.845281 & 1.641476 \\ \mathrm{H} & -7.482875 & -3.567228 & 1.846938 \\ \mathrm{H} & -6.614945 & -2.163777 & 2.495009 \\ \mathrm{H} & -5.752998 & -3.406393 & 1.590514\end{array}$




\begin{tabular}{|c|c|c|c|}
\hline $\mathrm{C}$ & -8.496984 & -1.630119 & 0.557903 \\
\hline $\mathrm{H}$ & -9.143108 & -2.513003 & 0.501961 \\
\hline $\mathrm{H}$ & -8.612891 & -1.202625 & 1.561367 \\
\hline $\mathrm{C}$ & -8.945073 & -0.600950 & -0.449435 \\
\hline $\mathrm{H}$ & -8.934607 & -1.010717 & -1.473408 \\
\hline $\mathrm{H}$ & -9.977561 & -0.295748 & -0.242156 \\
\hline $\mathrm{C}$ & -6.759591 & 0.447966 & -0.067710 \\
\hline $\mathrm{C}$ & -8.697604 & 1.743606 & -0.990741 \\
\hline $\mathrm{H}$ & -9.499884 & 2.122025 & -0.340817 \\
\hline $\mathrm{H}$ & -9.175527 & 1.431385 & -1.930374 \\
\hline $\mathrm{C}$ & -7.692280 & 2.842077 & -1.275210 \\
\hline $\mathrm{H}$ & -8.229970 & 3.779294 & -1.456909 \\
\hline $\mathrm{H}$ & -7.125361 & 2.609540 & -2.184369 \\
\hline $\mathrm{C}$ & -6.703947 & 3.005249 & -0.112775 \\
\hline $\mathrm{C}$ & -7.443629 & 3.325389 & 1.201999 \\
\hline $\mathrm{H}$ & -6.719451 & 3.498447 & 2.003710 \\
\hline $\mathrm{H}$ & -8.096092 & 2.506017 & 1.516130 \\
\hline $\mathrm{H}$ & -8.052592 & 4.229904 & 1.088296 \\
\hline $\mathrm{C}$ & -5.756905 & 4.164828 & -0.441163 \\
\hline $\mathrm{H}$ & -5.064642 & 4.379189 & 0.378491 \\
\hline $\mathrm{H}$ & -6.341973 & 5.074337 & -0.613577 \\
\hline $\mathrm{H}$ & -5.171213 & 3.960955 & -1.343378 \\
\hline $\mathrm{C}$ & -5.987385 & 1.668120 & 0.023432 \\
\hline $\mathrm{C}$ & -4.647959 & 1.591788 & 0.254953 \\
\hline $\mathrm{H}$ & -4.053970 & 2.495810 & 0.332298 \\
\hline $\mathrm{C}$ & -3.979466 & 0.351169 & 0.378634 \\
\hline $\mathrm{C}$ & -2.578677 & 0.329000 & 0.547649 \\
\hline $\mathrm{H}$ & -2.058859 & 1.280845 & 0.611952 \\
\hline $\mathrm{C}$ & 0.362171 & -0.866649 & -0.727660 \\
\hline $\mathrm{C}$ & -0.099951 & -2.059456 & -1.463900 \\
\hline $\mathrm{C}$ & -0.113462 & 0.323099 & -1.459846 \\
\hline $\mathrm{C}$ & 1.889946 & -0.857531 & -0.618340 \\
\hline $\mathrm{C}$ & 2.578677 & 0.328999 & -0.547648 \\
\hline $\mathrm{H}$ & 2.058859 & 1.280843 & -0.611956 \\
\hline $\mathrm{C}$ & 3.979466 & 0.351169 & -0.378636 \\
\hline $\mathrm{C}$ & 4.647959 & 1.591788 & -0.254959 \\
\hline $\mathrm{H}$ & 4.053970 & 2.495810 & -0.332305 \\
\hline $\mathrm{C}$ & 5.987385 & 1.668121 & -0.023440 \\
\hline $\mathrm{C}$ & 6.703948 & 3.005248 & 0.112772 \\
\hline $\mathrm{C}$ & 6.759591 & 0.447966 & 0.067701 \\
\hline
\end{tabular}




\begin{tabular}{|c|c|c|c|}
\hline $\mathrm{C}$ & 8.945073 & -0.600945 & 0.449425 \\
\hline $\mathrm{H}$ & 8.934601 & -1.010708 & 1.473400 \\
\hline $\mathrm{H}$ & 9.977563 & -0.295746 & 0.242150 \\
\hline $\mathrm{C}$ & 8.496986 & -1.630117 & -0.557910 \\
\hline $\mathrm{H}$ & 9.143110 & -2.513001 & -0.501965 \\
\hline $\mathrm{H}$ & 8.612894 & -1.202626 & -1.561376 \\
\hline $\mathrm{C}$ & 7.032470 & -2.060353 & -0.355007 \\
\hline $\mathrm{C}$ & 6.684507 & -2.845276 & -1.641488 \\
\hline $\mathrm{H}$ & 5.753006 & -3.406394 & -1.590528 \\
\hline $\mathrm{H}$ & 7.482883 & -3.567217 & -1.846955 \\
\hline $\mathrm{H}$ & 6.614943 & -2.163768 & -2.495018 \\
\hline $\mathrm{C}$ & 6.953817 & -2.983537 & 0.877975 \\
\hline $\mathrm{H}$ & 5.931794 & -3.295314 & 1.101228 \\
\hline $\mathrm{H}$ & 7.333107 & -2.482050 & 1.772917 \\
\hline $\mathrm{H}$ & 7.552591 & -3.886707 & 0.711048 \\
\hline $\mathrm{C}$ & 6.161803 & -0.798533 & -0.188619 \\
\hline $\mathrm{C}$ & 4.734812 & -0.853549 & -0.324380 \\
\hline $\mathrm{C}$ & 3.973151 & -2.059800 & -0.368440 \\
\hline $\mathrm{H}$ & 4.457763 & -3.016763 & -0.257559 \\
\hline $\mathrm{C}$ & 2.609482 & -2.068436 & -0.509265 \\
\hline $\mathrm{H}$ & 2.088085 & -3.019981 & -0.527725 \\
\hline $\mathrm{N}$ & 8.106845 & 0.571669 & 0.360169 \\
\hline $\mathrm{C}$ & 7.443646 & 3.325389 & -1.201994 \\
\hline $\mathrm{H}$ & 8.052602 & 4.229907 & -1.088283 \\
\hline $\mathrm{H}$ & 6.719478 & 3.498442 & -2.003714 \\
\hline $\mathrm{H}$ & 8.096118 & 2.506019 & -1.516114 \\
\hline $\mathrm{C}$ & 5.756905 & 4.164829 & 0.441149 \\
\hline $\mathrm{H}$ & 6.341971 & 5.074337 & 0.613568 \\
\hline $\mathrm{H}$ & 5.171203 & 3.960958 & 1.343359 \\
\hline $\mathrm{H}$ & 5.064650 & 4.379188 & -0.378512 \\
\hline $\mathrm{C}$ & 7.692267 & 2.842073 & 1.275218 \\
\hline $\mathrm{H}$ & 8.229957 & 3.779289 & 1.456924 \\
\hline $\mathrm{C}$ & 8.697593 & 1.743598 & 0.990762 \\
\hline $\mathrm{H}$ & 9.499895 & 2.122022 & 0.340869 \\
\hline $\mathrm{H}$ & 9.175485 & 1.431360 & 1.930406 \\
\hline $\mathrm{H}$ & 7.125336 & 2.609537 & 2.184370 \\
\hline
\end{tabular}

$1 \pi$-dimer singlet $\mathrm{E}(\mathrm{UB} 98)=-2109.50922636$ a.u.; triplet $\mathrm{E}(\mathrm{UB} 98)=-2109.50602403$ a.u. Atomic Coordinates(Angstroms) $\begin{array}{llll}\text { type } & \mathrm{X} & \mathrm{Y} & \mathrm{Z}\end{array}$ 


\begin{tabular}{|c|c|c|c|}
\hline $\mathrm{N}$ & 1.750568 & -4.021268 & 1.389796 \\
\hline $\mathrm{N}$ & 3.326424 & -0.191110 & 2.867466 \\
\hline $\mathrm{C}$ & -2.536637 & 0.295679 & 1.120682 \\
\hline $\mathrm{C}$ & -2.262480 & -1.109809 & 0.867143 \\
\hline $\mathrm{C}$ & -1.005326 & -1.664936 & 1.080795 \\
\hline $\mathrm{C}$ & 0.085764 & -0.901671 & 1.575740 \\
\hline $\mathrm{C}$ & -0.167163 & 0.493232 & 1.824603 \\
\hline $\mathrm{C}$ & -1.398543 & 1.047662 & 1.614786 \\
\hline $\mathrm{C}$ & 1.347392 & -1.495078 & 1.817772 \\
\hline $\mathrm{C}$ & 1.574814 & -2.882286 & 1.582555 \\
\hline $\mathrm{C}$ & 2.432537 & -0.769588 & 2.385724 \\
\hline $\mathrm{H}$ & 0.646348 & 1.118955 & 2.180658 \\
\hline $\mathrm{H}$ & -0.862738 & -2.724589 & 0.883410 \\
\hline $\mathrm{N}$ & -1.428959 & 3.506655 & -1.473527 \\
\hline $\mathrm{N}$ & -3.309664 & -0.196655 & -2.740476 \\
\hline $\mathrm{C}$ & 2.560090 & -1.013333 & -1.252374 \\
\hline $\mathrm{C}$ & 2.504244 & 0.420206 & -1.075902 \\
\hline $\mathrm{C}$ & 1.240823 & 1.019642 & -1.298762 \\
\hline $\mathrm{C}$ & 0.102774 & 0.297720 & -1.713231 \\
\hline $\mathrm{C}$ & 0.212886 & -1.120744 & -1.925620 \\
\hline $\mathrm{C}$ & 1.414741 & -1.737484 & -1.701619 \\
\hline $\mathrm{C}$ & -1.153186 & 0.967331 & -1.908864 \\
\hline $\mathrm{C}$ & -1.298437 & 2.361204 & -1.678269 \\
\hline $\mathrm{C}$ & -2.318831 & 0.310749 & -2.374316 \\
\hline $\mathrm{H}$ & -0.651106 & -1.684362 & -2.265957 \\
\hline $\mathrm{H}$ & 1.108105 & 2.079973 & -1.147731 \\
\hline $\mathrm{C}$ & -3.835035 & 0.810259 & 0.888479 \\
\hline $\mathrm{C}$ & -4.547764 & -1.481623 & 0.070891 \\
\hline $\mathrm{C}$ & -4.808639 & -0.050861 & 0.240178 \\
\hline $\mathrm{C}$ & -3.312889 & -1.951793 & 0.398879 \\
\hline $\mathrm{C}$ & 3.772308 & -1.690089 & -1.002859 \\
\hline $\mathrm{C}$ & 3.689610 & 1.135743 & -0.659878 \\
\hline $\mathrm{C}$ & 4.844118 & 0.390700 & -0.316448 \\
\hline $\mathrm{C}$ & 4.895582 & -1.050939 & -0.533004 \\
\hline $\mathrm{H}$ & 1.511587 & -2.811992 & -1.840919 \\
\hline $\mathrm{H}$ & -1.490263 & 2.103272 & 1.796639 \\
\hline $\mathrm{H}$ & -3.082661 & -3.005100 & 0.273505 \\
\hline $\mathrm{C}$ & -5.631161 & -2.446517 & -0.433126 \\
\hline $\mathrm{C}$ & -4.365278 & 2.200447 & 1.332773 \\
\hline $\mathrm{C}$ & -6.544538 & -1.664660 & -1.393233 \\
\hline
\end{tabular}




\begin{tabular}{|c|c|c|c|}
\hline $\mathrm{H}$ & -5.973498 & -1.392632 & -2.289690 \\
\hline $\mathrm{H}$ & -7.390607 & -2.290043 & -1.705539 \\
\hline $\mathrm{C}$ & -7.076298 & -0.403584 & -0.736190 \\
\hline $\mathrm{H}$ & -7.622252 & 0.197624 & -1.471396 \\
\hline $\mathrm{H}$ & -7.787918 & -0.640066 & 0.070737 \\
\hline $\mathrm{C}$ & -6.370746 & 1.857174 & -0.174802 \\
\hline $\mathrm{H}$ & -7.187456 & 1.993272 & 0.552141 \\
\hline $\mathrm{H}$ & -6.778446 & 2.112325 & -1.161743 \\
\hline $\mathrm{N}$ & -6.003602 & 0.441775 & -0.198876 \\
\hline $\mathrm{C}$ & -5.198175 & 2.755807 & 0.154579 \\
\hline $\mathrm{H}$ & -4.544203 & 2.869910 & -0.718203 \\
\hline $\mathrm{H}$ & -5.584352 & 3.751776 & 0.403373 \\
\hline $\mathrm{N}$ & 5.978374 & 1.001171 & 0.214081 \\
\hline $\mathrm{C}$ & 3.698783 & 2.688456 & -0.598162 \\
\hline $\mathrm{C}$ & 5.141294 & 3.193783 & -0.343304 \\
\hline $\mathrm{H}$ & 5.101651 & 4.247261 & -0.038586 \\
\hline $\mathrm{H}$ & 5.723415 & 3.142555 & -1.273036 \\
\hline $\mathrm{C}$ & 5.858233 & 2.363932 & 0.705265 \\
\hline $\mathrm{H}$ & 6.869813 & 2.750860 & 0.875692 \\
\hline $\mathrm{H}$ & 5.328522 & 2.387487 & 1.673953 \\
\hline $\mathrm{C}$ & 6.140890 & -1.907693 & -0.227011 \\
\hline $\mathrm{C}$ & 7.360516 & -0.999587 & 0.028902 \\
\hline $\mathrm{H}$ & 7.753635 & -0.623725 & -0.925585 \\
\hline $\mathrm{H}$ & 8.158108 & -1.581458 & 0.508566 \\
\hline $\mathrm{C}$ & 6.984214 & 0.192094 & 0.894072 \\
\hline $\mathrm{H}$ & 6.625311 & -0.130685 & 1.886839 \\
\hline $\mathrm{H}$ & 7.859408 & 0.831159 & 1.058092 \\
\hline $\mathrm{H}$ & 3.789646 & -2.765203 & -1.159064 \\
\hline $\mathrm{C}$ & 5.861188 & -2.779896 & 1.028536 \\
\hline $\mathrm{H}$ & 5.002847 & -3.440577 & 0.865053 \\
\hline $\mathrm{H}$ & 6.736255 & -3.404535 & 1.251712 \\
\hline $\mathrm{H}$ & 5.638760 & -2.167102 & 1.908518 \\
\hline $\mathrm{C}$ & 6.483602 & -2.840211 & -1.415741 \\
\hline $\mathrm{H}$ & 7.424007 & -3.366408 & -1.207162 \\
\hline $\mathrm{H}$ & 5.715262 & -3.601562 & -1.585647 \\
\hline $\mathrm{H}$ & 6.609301 & -2.267559 & -2.342001 \\
\hline $\mathrm{C}$ & 3.290816 & 3.330036 & -1.956293 \\
\hline $\mathrm{H}$ & 2.236628 & 3.215961 & -2.212681 \\
\hline $\mathrm{H}$ & 3.500555 & 4.407024 & -1.920424 \\
\hline $\mathrm{H}$ & 3.882067 & 2.897248 & -2.771818 \\
\hline
\end{tabular}




$\begin{array}{lrrr}\mathrm{C} & 2.807161 & 3.235721 & 0.552731 \\ \mathrm{H} & 1.763678 & 2.922952 & 0.475873 \\ \mathrm{H} & 3.175333 & 2.895235 & 1.526663 \\ \mathrm{H} & 2.824101 & 4.333549 & 0.541526 \\ \mathrm{C} & -5.257837 & 1.973027 & 2.588775 \\ \mathrm{H} & -6.025015 & 1.207512 & 2.432246 \\ \mathrm{H} & -4.638105 & 1.648971 & 3.432349 \\ \mathrm{H} & -5.757921 & 2.908467 & 2.873197 \\ \mathrm{C} & -3.367168 & 3.326456 & 1.694004 \\ \mathrm{H} & -2.832146 & 3.136405 & 2.628697 \\ \mathrm{H} & -2.656058 & 3.536123 & 0.887975 \\ \mathrm{H} & -3.951969 & 4.240659 & 1.855147 \\ \mathrm{C} & -6.430660 & -2.990586 & 0.781931 \\ \mathrm{H} & -7.254983 & -3.630788 & 0.441200 \\ \mathrm{H} & -5.777578 & -3.588377 & 1.427713 \\ \mathrm{H} & -6.851046 & -2.188630 & 1.398834 \\ \mathrm{C} & -5.042232 & -3.642009 & -1.215328 \\ \mathrm{H} & -4.475951 & -4.325748 & -0.573786 \\ \mathrm{H} & -5.862096 & -4.222945 & -1.655301 \\ \mathrm{H} & -4.390107 & -3.298917 & -2.026054\end{array}$

$1 \pi$-dimer singlet $\mathrm{E}(\mathrm{UB} 97 \mathrm{D})=--2108.89606900$ a.u.; triplet $\mathrm{E}(\mathrm{UB} 97 \mathrm{D})=-2108.88702214$ a.u.

\begin{tabular}{lrrr} 
Atomic & \multicolumn{3}{c}{ Coordinates(Angstroms) } \\
type & $\mathrm{X}$ & $\mathrm{Y}$ & \multicolumn{1}{c}{$\mathrm{Z}$} \\
$\mathrm{N}$ & 1.969739 & -3.917348 & 1.388427 \\
$\mathrm{~N}$ & 3.558814 & 0.022673 & 2.546563 \\
$\mathrm{C}$ & -2.384535 & 0.368632 & 1.041797 \\
$\mathrm{C}$ & -2.123425 & -1.055844 & 0.871140 \\
$\mathrm{C}$ & -0.848530 & -1.597800 & 1.063663 \\
$\mathrm{C}$ & 0.260337 & -0.797015 & 1.441795 \\
$\mathrm{C}$ & 0.025893 & 0.617747 & 1.566488 \\
$\mathrm{C}$ & -1.220301 & 1.161462 & 1.378682 \\
$\mathrm{C}$ & 1.548008 & -1.364962 & 1.662238 \\
$\mathrm{C}$ & 1.784431 & -2.761592 & 1.516598 \\
$\mathrm{C}$ & 2.645300 & -0.597138 & 2.136111 \\
$\mathrm{H}$ & 0.867389 & 1.271625 & 1.788045 \\
$\mathrm{H}$ & -0.713752 & -2.672159 & 0.936123 \\
$\mathrm{~N}$ & -1.716117 & 3.251225 & -1.511273 \\
$\mathrm{~N}$ & -3.441748 & -0.566347 & -2.655591 \\
$\mathrm{C}$ & 2.486322 & -1.106241 & -1.209584
\end{tabular}




\begin{tabular}{|c|c|c|c|}
\hline $\mathrm{C}$ & 2.381891 & 0.339484 & -1.106466 \\
\hline $\mathrm{C}$ & 1.091732 & 0.882929 & -1.339068 \\
\hline $\mathrm{C}$ & -0.028078 & 0.099511 & -1.683793 \\
\hline $\mathrm{C}$ & 0.126201 & -1.328617 & -1.813260 \\
\hline $\mathrm{C}$ & 1.359366 & -1.895227 & -1.593284 \\
\hline $\mathrm{C}$ & -1.320974 & 0.707519 & -1.870429 \\
\hline $\mathrm{C}$ & -1.530964 & 2.098537 & -1.681167 \\
\hline $\mathrm{C}$ & -2.463799 & -0.008102 & -2.302414 \\
\hline $\mathrm{H}$ & -0.733616 & -1.941036 & -2.082644 \\
\hline $\mathrm{H}$ & 0.921854 & 1.945229 & -1.234734 \\
\hline $\mathrm{C}$ & -3.703838 & 0.861156 & 0.862011 \\
\hline $\mathrm{C}$ & -4.433136 & -1.460346 & 0.161109 \\
\hline $\mathrm{C}$ & -4.690784 & -0.026650 & 0.279586 \\
\hline $\mathrm{C}$ & -3.188530 & -1.922031 & 0.494870 \\
\hline $\mathrm{C}$ & 3.724447 & -1.732163 & -0.935889 \\
\hline $\mathrm{C}$ & 3.538480 & 1.111204 & -0.732049 \\
\hline $\mathrm{C}$ & 4.720932 & 0.419038 & -0.345827 \\
\hline $\mathrm{C}$ & 4.825238 & -1.030141 & -0.498131 \\
\hline $\mathrm{H}$ & 1.490357 & -2.975910 & -1.661035 \\
\hline $\mathrm{H}$ & -1.287196 & 2.236668 & 1.417277 \\
\hline $\mathrm{H}$ & -2.964338 & -2.984811 & 0.420276 \\
\hline $\mathrm{C}$ & -5.526946 & -2.439577 & -0.278039 \\
\hline $\mathrm{C}$ & -4.245700 & 2.240760 & 1.306388 \\
\hline $\mathrm{C}$ & -6.491678 & -1.699561 & -1.223937 \\
\hline $\mathrm{H}$ & -5.959377 & -1.458037 & -2.154919 \\
\hline $\mathrm{H}$ & -7.347792 & -2.348587 & -1.463709 \\
\hline $\mathrm{C}$ & -7.001096 & -0.413461 & -0.590810 \\
\hline $\mathrm{H}$ & -7.597513 & 0.153408 & -1.319843 \\
\hline $\mathrm{H}$ & -7.656701 & -0.624002 & 0.274001 \\
\hline $\mathrm{C}$ & -6.259384 & 1.881072 & -0.179447 \\
\hline $\mathrm{H}$ & -7.071480 & 2.038680 & 0.553884 \\
\hline $\mathrm{H}$ & -6.672349 & 2.103067 & -1.176855 \\
\hline $\mathrm{N}$ & -5.899262 & 0.457807 & -0.154356 \\
\hline $\mathrm{C}$ & -5.082993 & 2.791477 & 0.127337 \\
\hline $\mathrm{H}$ & -4.426769 & 2.895654 & -0.747849 \\
\hline $\mathrm{H}$ & -5.473082 & 3.790037 & 0.376304 \\
\hline $\mathrm{N}$ & 5.819830 & 1.089699 & 0.181857 \\
\hline $\mathrm{C}$ & 3.489943 & 2.661047 & -0.715761 \\
\hline $\mathrm{C}$ & 4.912389 & 3.230417 & -0.473848 \\
\hline $\mathrm{H}$ & 4.830606 & 4.297421 & -0.216769 \\
\hline
\end{tabular}




\begin{tabular}{|c|c|c|c|}
\hline $\mathrm{H}$ & 5.507829 & 3.150845 & -1.397313 \\
\hline $\mathrm{C}$ & 5.641428 & 2.469520 & 0.620908 \\
\hline $\mathrm{H}$ & 6.638292 & 2.899469 & 0.797847 \\
\hline $\mathrm{H}$ & 5.083668 & 2.496237 & 1.576378 \\
\hline $\mathrm{C}$ & 6.085409 & -1.825418 & -0.117882 \\
\hline $\mathrm{C}$ & 7.277303 & -0.870828 & 0.095405 \\
\hline $\mathrm{H}$ & 7.659288 & -0.525601 & -0.879159 \\
\hline $\mathrm{H}$ & 8.089429 & -1.408812 & 0.608380 \\
\hline $\mathrm{C}$ & 6.852947 & 0.345733 & 0.906345 \\
\hline $\mathrm{H}$ & 6.479494 & 0.049510 & 1.904388 \\
\hline $\mathrm{H}$ & 7.704086 & 1.026514 & 1.052866 \\
\hline $\mathrm{H}$ & 3.774913 & -2.816428 & -1.023976 \\
\hline $\mathrm{C}$ & 5.798815 & -2.608475 & 1.193125 \\
\hline $\mathrm{H}$ & 4.958111 & -3.301586 & 1.051399 \\
\hline $\mathrm{H}$ & 6.692005 & -3.188030 & 1.477753 \\
\hline $\mathrm{H}$ & 5.537911 & -1.928566 & 2.015003 \\
\hline $\mathrm{C}$ & 6.474795 & -2.833583 & -1.227200 \\
\hline $\mathrm{H}$ & 7.422052 & -3.323643 & -0.951632 \\
\hline $\mathrm{H}$ & 5.717347 & -3.619175 & -1.356114 \\
\hline $\mathrm{H}$ & 6.612709 & -2.318509 & -2.189825 \\
\hline $\mathrm{C}$ & 3.059778 & 3.253077 & -2.089232 \\
\hline $\mathrm{H}$ & 2.018959 & 3.058741 & -2.365648 \\
\hline $\mathrm{H}$ & 3.195233 & 4.346410 & -2.060824 \\
\hline $\mathrm{H}$ & 3.702924 & 2.846379 & -2.884233 \\
\hline $\mathrm{C}$ & 2.582725 & 3.200867 & 0.426548 \\
\hline $\mathrm{H}$ & 1.540533 & 2.876159 & 0.342396 \\
\hline $\mathrm{H}$ & 2.957265 & 2.860890 & 1.402063 \\
\hline $\mathrm{H}$ & 2.593823 & 4.302763 & 0.408149 \\
\hline $\mathrm{C}$ & -5.145656 & 1.986587 & 2.554823 \\
\hline $\mathrm{H}$ & -5.895061 & 1.202531 & 2.378582 \\
\hline $\mathrm{H}$ & -4.519044 & 1.671895 & 3.401915 \\
\hline $\mathrm{H}$ & -5.667973 & 2.916805 & 2.832060 \\
\hline $\mathrm{C}$ & -3.266492 & 3.368478 & 1.698968 \\
\hline $\mathrm{H}$ & -2.670765 & 3.114214 & 2.585886 \\
\hline $\mathrm{H}$ & -2.614431 & 3.655908 & 0.862996 \\
\hline $\mathrm{H}$ & -3.876386 & 4.247162 & 1.961912 \\
\hline $\mathrm{C}$ & -6.273113 & -2.944960 & 0.985722 \\
\hline $\mathrm{H}$ & -7.101389 & -3.610957 & 0.693713 \\
\hline $\mathrm{H}$ & -5.580575 & -3.505904 & 1.630294 \\
\hline $\mathrm{H}$ & -6.683162 & -2.114440 & 1.578861 \\
\hline
\end{tabular}




$\begin{array}{llll}\mathrm{C} & -4.964750 & -3.654776 & -1.049789 \\ \mathrm{H} & -4.367440 & -4.315418 & -0.405143 \\ \mathrm{H} & -5.805502 & -4.249519 & -1.439271 \\ \mathrm{H} & -4.345614 & -3.317520 & -1.892768\end{array}$

$1 \pi$-dimer singlet $\mathrm{E}(\mathrm{U} \omega \mathrm{B} 97 \mathrm{XD})=-2109.73413250$ a.u.; triplet $\mathrm{E}(\mathrm{U} \omega \mathrm{B} 97 \mathrm{XD})=-2109.72856693$ a.u.

\begin{tabular}{lrrr} 
Atomic & \multicolumn{3}{c}{ Coordinates(Angstroms) } \\
type & $\mathrm{X}$ & $\mathrm{Y}$ & $\mathrm{Z}$ \\
$\mathrm{N}$ & 1.937242 & -3.857996 & 1.276254 \\
$\mathrm{~N}$ & 3.479875 & 0.027463 & 2.629559 \\
$\mathrm{C}$ & -2.393169 & 0.346436 & 1.046121 \\
$\mathrm{C}$ & -2.103536 & -1.059973 & 0.832826 \\
$\mathrm{C}$ & -0.841408 & -1.577055 & 0.999205 \\
$\mathrm{C}$ & 0.257133 & -0.774019 & 1.421073 \\
$\mathrm{C}$ & -0.010778 & 0.626126 & 1.624399 \\
$\mathrm{C}$ & -1.250360 & 1.143224 & 1.452874 \\
$\mathrm{C}$ & 1.515207 & -1.329545 & 1.633993 \\
$\mathrm{C}$ & 1.756735 & -2.721589 & 1.438210 \\
$\mathrm{C}$ & 2.599217 & -0.573129 & 2.166876 \\
$\mathrm{H}$ & 0.809841 & 1.277414 & 1.905353 \\
$\mathrm{H}$ & -0.682455 & -2.639367 & 0.839951 \\
$\mathrm{~N}$ & -1.574465 & 3.334851 & -1.346172 \\
$\mathrm{~N}$ & -3.434445 & -0.368167 & -2.596470 \\
$\mathrm{C}$ & 2.478363 & -1.061699 & -1.280894 \\
$\mathrm{C}$ & 2.385588 & 0.354198 & -1.100351 \\
$\mathrm{C}$ & 1.107557 & 0.916376 & -1.281250 \\
$\mathrm{C}$ & -0.014176 & 0.171824 & -1.664021 \\
$\mathrm{C}$ & 0.129439 & -1.236507 & -1.880361 \\
$\mathrm{C}$ & 1.350669 & -1.815803 & -1.702293 \\
$\mathrm{C}$ & -1.285511 & 0.807585 & -1.796416 \\
$\mathrm{C}$ & -1.441204 & 2.196596 & -1.559997 \\
$\mathrm{C}$ & -2.444370 & 0.143767 & -2.251210 \\
$\mathrm{H}$ & -0.730016 & -1.824686 & -2.186957 \\
$\mathrm{H}$ & 0.948257 & 1.968564 & -1.114824 \\
$\mathrm{C}$ & -3.697351 & 0.820860 & 0.858002 \\
$\mathrm{C}$ & -4.405394 & -1.501707 & 0.180545 \\
$\mathrm{C}$ & -4.647382 & -0.062677 & 0.225783 \\
$\mathrm{C}$ & -3.165557 & -1.943792 & 0.476493 \\
$\mathrm{C}$ & 3.707210 & -1.703973 & -1.029490
\end{tabular}




\begin{tabular}{|c|c|c|c|}
\hline $\mathrm{C}$ & 3.547377 & 1.101199 & -0.694241 \\
\hline $\mathrm{C}$ & 4.708435 & 0.395734 & -0.342950 \\
\hline $\mathrm{C}$ & 4.793991 & -1.039692 & -0.542239 \\
\hline $\mathrm{H}$ & 1.473411 & -2.886056 & -1.841808 \\
\hline $\mathrm{H}$ & -1.356146 & 2.205674 & 1.571401 \\
\hline $\mathrm{H}$ & -2.934720 & -3.001615 & 0.422165 \\
\hline $\mathrm{C}$ & -5.536940 & -2.455341 & -0.171832 \\
\hline $\mathrm{C}$ & -4.270936 & 2.189579 & 1.267112 \\
\hline $\mathrm{C}$ & -6.343025 & -1.792167 & -1.291464 \\
\hline $\mathrm{H}$ & -5.704520 & -1.684302 & -2.173683 \\
\hline $\mathrm{H}$ & -7.202540 & -2.415078 & -1.562898 \\
\hline $\mathrm{C}$ & -6.845086 & -0.431448 & -0.866333 \\
\hline $\mathrm{H}$ & -7.248196 & 0.098077 & -1.734098 \\
\hline $\mathrm{H}$ & -7.662385 & -0.516335 & -0.136179 \\
\hline $\mathrm{C}$ & -6.132158 & 1.830956 & -0.383781 \\
\hline $\mathrm{H}$ & -7.019498 & 2.008485 & 0.240028 \\
\hline $\mathrm{H}$ & -6.420953 & 2.035574 & -1.420204 \\
\hline $\mathrm{N}$ & -5.798480 & 0.413327 & -0.291923 \\
\hline $\mathrm{C}$ & -4.995299 & 2.734538 & 0.026838 \\
\hline $\mathrm{H}$ & -4.268000 & 2.839199 & -0.784109 \\
\hline $\mathrm{H}$ & -5.401494 & 3.731152 & 0.229296 \\
\hline $\mathrm{N}$ & 5.821450 & 1.034260 & 0.191052 \\
\hline $\mathrm{C}$ & 3.506318 & 2.643865 & -0.628946 \\
\hline $\mathrm{C}$ & 4.930572 & 3.179727 & -0.400996 \\
\hline $\mathrm{H}$ & 4.875343 & 4.238568 & -0.126325 \\
\hline $\mathrm{H}$ & 5.504226 & 3.111529 & -1.332971 \\
\hline $\mathrm{C}$ & 5.663120 & 2.393493 & 0.658340 \\
\hline $\mathrm{H}$ & 6.660605 & 2.813742 & 0.821756 \\
\hline $\mathrm{H}$ & 5.131533 & 2.418258 & 1.623729 \\
\hline $\mathrm{C}$ & 6.030292 & -1.866363 & -0.167422 \\
\hline $\mathrm{C}$ & 7.220556 & -0.943742 & 0.114193 \\
\hline $\mathrm{H}$ & 7.645750 & -0.583250 & -0.830393 \\
\hline $\mathrm{H}$ & 8.003745 & -1.504959 & 0.636287 \\
\hline $\mathrm{C}$ & 6.796800 & 0.255317 & 0.932051 \\
\hline $\mathrm{H}$ & 6.394294 & -0.047475 & 1.912079 \\
\hline $\mathrm{H}$ & 7.657653 & 0.903691 & 1.121172 \\
\hline $\mathrm{H}$ & 3.751175 & -2.778956 & -1.173865 \\
\hline $\mathrm{C}$ & 5.700276 & -2.693908 & 1.093086 \\
\hline $\mathrm{H}$ & 4.866275 & -3.376760 & 0.905814 \\
\hline $\mathrm{H}$ & 6.570843 & -3.290394 & 1.390291 \\
\hline
\end{tabular}




$\begin{array}{lrrr}\text { H } & 5.415948 & -2.053187 & 1.932755 \\ \mathrm{C} & 6.429138 & -2.822874 & -1.304430 \\ \mathrm{H} & 7.372583 & -3.319000 & -1.050329 \\ \mathrm{H} & 5.684822 & -3.606502 & -1.470372 \\ \mathrm{H} & 6.569464 & -2.279697 & -2.244181 \\ \mathrm{C} & 3.053604 & 3.272534 & -1.966641 \\ \mathrm{H} & 1.994271 & 3.147674 & -2.188204 \\ \mathrm{H} & 3.251418 & 4.350206 & -1.940914 \\ \mathrm{H} & 3.621410 & 2.842671 & -2.797781 \\ \mathrm{C} & 2.624298 & 3.154145 & 0.533375 \\ \mathrm{H} & 1.566913 & 2.920029 & 0.402900 \\ \mathrm{H} & 2.944345 & 2.720047 & 1.485168 \\ \mathrm{H} & 2.704837 & 4.244832 & 0.606571 \\ \mathrm{C} & -5.266427 & 1.938442 & 2.426544 \\ \mathrm{H} & -5.997273 & 1.156703 & 2.200425 \\ \mathrm{H} & -4.721801 & 1.629699 & 3.323763 \\ \mathrm{H} & -5.813410 & 2.859318 & 2.659668 \\ \mathrm{C} & -3.320573 & 3.297822 & 1.738701 \\ \mathrm{H} & -2.788481 & 3.032381 & 2.655421 \\ \mathrm{H} & -2.613207 & 3.596238 & 0.960500 \\ \mathrm{H} & -3.931431 & 4.176270 & 1.973292 \\ \mathrm{C} & -6.402584 & -2.678849 & 1.084978 \\ \mathrm{H} & -7.287593 & -3.276730 & 0.838491 \\ \mathrm{H} & -5.827914 & -3.212095 & 1.848091 \\ \mathrm{H} & -6.739485 & -1.737686 & 1.530882 \\ \mathrm{C} & -5.032951 & -3.811108 & -0.678851 \\ \mathrm{H} & -4.519361 & -4.381137 & 0.101202 \\ \mathrm{H} & -5.886159 & -4.413542 & -1.007135 \\ \mathrm{H} & -4.354487 & -3.687662 & -1.528576\end{array}$

2 radical $\mathrm{E}(\mathrm{U} \omega \mathrm{B} 97 \mathrm{XD})=-901.27028391$ a.u

\begin{tabular}{crrr} 
Atomic & \multicolumn{3}{c}{ Coordinates(Angstroms) } \\
type & $\mathrm{X}$ & $\mathrm{Y}$ & $\mathrm{Z}$ \\
$\mathrm{N}$ & 2.400288 & 0.000026 & -0.143377 \\
$\mathrm{~N}$ & -4.528436 & -2.229935 & 0.009075 \\
$\mathrm{~N}$ & -4.528283 & 2.229909 & 0.008804 \\
$\mathrm{C}$ & 1.030739 & 0.000003 & -0.085589 \\
$\mathrm{C}$ & 0.304509 & -1.236277 & -0.064365 \\
$\mathrm{C}$ & -1.069743 & -1.207126 & -0.042715 \\
$\mathrm{C}$ & -1.805216 & -0.000039 & -0.036079
\end{tabular}




$\begin{array}{lrrr}\text { C } & -1.069776 & 1.207068 & -0.042682 \\ \mathrm{C} & 0.304476 & 1.236262 & -0.064343 \\ \mathrm{C} & -3.227403 & -0.000056 & -0.014384 \\ \mathrm{C} & -3.961918 & -1.213225 & -0.001588 \\ \mathrm{C} & -3.961950 & 1.213093 & -0.001542 \\ \mathrm{C} & 1.035588 & -2.577344 & 0.006425 \\ \mathrm{C} & 2.427675 & -2.404537 & -0.613706 \\ \mathrm{H} & 3.016703 & -3.315792 & -0.464415 \\ \mathrm{H} & 2.325024 & -2.252692 & -1.694975 \\ \mathrm{C} & 3.170077 & -1.226427 & -0.018574 \\ \mathrm{H} & 3.420429 & -1.411080 & 1.037218 \\ \mathrm{H} & 4.117592 & -1.078463 & -0.546205 \\ \mathrm{C} & 3.170010 & 1.226498 & -0.018361 \\ \mathrm{H} & 4.117616 & 1.078607 & -0.545844 \\ \mathrm{H} & 3.420178 & 1.411093 & 1.037485 \\ \mathrm{C} & 2.427648 & 2.404608 & -0.613545 \\ \mathrm{H} & 3.016633 & 3.315877 & -0.464170 \\ \mathrm{H} & 2.325122 & 2.252779 & -1.694828 \\ \mathrm{C} & 1.035493 & 2.577368 & 0.006442 \\ \mathrm{H} & -1.619430 & 2.141672 & -0.022067 \\ \mathrm{H} & -1.619370 & -2.141746 & -0.022123 \\ \mathrm{C} & 0.305910 & -3.683061 & -0.772225 \\ \mathrm{H} & -0.644523 & -3.961842 & -0.310119 \\ \mathrm{H} & 0.108504 & -3.378227 & -1.804503 \\ \mathrm{H} & 0.927803 & -4.584467 & -0.793486 \\ \mathrm{C} & 1.146544 & -3.002294 & 1.484619 \\ \mathrm{H} & 0.150279 & -3.157113 & 1.909194 \\ \mathrm{H} & 1.706492 & -3.940572 & 1.572285 \\ \mathrm{H} & 1.644909 & -2.244058 & 2.095889 \\ \mathrm{C} & 0.305829 & 3.683002 & -0.772346 \\ \mathrm{H} & 0.108515 & 3.378080 & -1.804615 \\ \mathrm{H} & -0.644650 & 3.961778 & -0.310333 \\ \mathrm{H} & 0.927688 & 4.584431 & -0.793625 \\ \mathrm{C} & 1.146290 & 3.002412 & 1.484620 \\ \mathrm{H} & 1.706137 & 3.940752 & 1.572276 \\ \mathrm{H} & 0.149979 & 3.157156 & 1.909114 \\ \mathrm{H} & 1.644688 & 2.244261 & 2.095969 \\ & & & \end{array}$

$2 \sigma$-dimer $\mathrm{E}(\mathrm{RB} 98)=-1802.37380117$ a.u. 


\begin{tabular}{|c|c|c|c|}
\hline \multirow{2}{*}{$\begin{array}{l}\text { Atomic } \\
\text { type }\end{array}$} & \multicolumn{3}{|c|}{ Coordinates(Angstroms) } \\
\hline & $\mathrm{X}$ & $\mathrm{Y}$ & Z \\
\hline $\mathrm{N}$ & -1.312267 & 5.145804 & 2.971796 \\
\hline $\mathrm{N}$ & 2.978119 & 0.291217 & -0.082549 \\
\hline $\mathrm{N}$ & 0.161474 & -1.833922 & 2.359654 \\
\hline $\mathrm{C}$ & -0.966353 & 1.519693 & 2.325689 \\
\hline $\mathrm{H}$ & -1.396934 & 0.584399 & 2.666703 \\
\hline $\mathrm{C}$ & -1.417523 & 2.710285 & 2.890560 \\
\hline $\mathrm{C}$ & -2.476441 & 2.711508 & 4.000414 \\
\hline $\mathrm{C}$ & -3.404023 & 1.478425 & 3.946074 \\
\hline $\mathrm{H}$ & -3.859385 & 1.359464 & 2.956117 \\
\hline $\mathrm{H}$ & -4.208262 & 1.594586 & 4.683181 \\
\hline $\mathrm{H}$ & -2.874723 & 0.551276 & 4.192369 \\
\hline $\mathrm{C}$ & -1.774508 & 2.747682 & 5.384263 \\
\hline $\mathrm{H}$ & -1.205505 & 1.824520 & 5.542295 \\
\hline $\mathrm{H}$ & -2.515440 & 2.835594 & 6.190514 \\
\hline $\mathrm{H}$ & -1.072594 & 3.584381 & 5.468207 \\
\hline $\mathrm{C}$ & -3.341755 & 3.976265 & 3.791246 \\
\hline $\mathrm{H}$ & -3.864925 & 3.888969 & 2.829617 \\
\hline $\mathrm{H}$ & -4.107779 & 4.047309 & 4.574185 \\
\hline $\mathrm{C}$ & -2.503219 & 5.249638 & 3.808265 \\
\hline $\mathrm{H}$ & -3.106556 & 6.094810 & 3.441959 \\
\hline $\mathrm{H}$ & -2.206566 & 5.503431 & 4.839724 \\
\hline $\mathrm{C}$ & -0.864289 & 3.949958 & 2.441405 \\
\hline $\mathrm{C}$ & -0.657964 & 6.423139 & 2.714922 \\
\hline $\mathrm{H}$ & -0.591796 & 6.970533 & 3.667837 \\
\hline $\mathrm{H}$ & -1.288011 & 7.035104 & 2.047604 \\
\hline $\mathrm{C}$ & 0.737096 & 6.250960 & 2.124794 \\
\hline $\mathrm{H}$ & 1.421169 & 5.878732 & 2.899594 \\
\hline $\mathrm{H}$ & 1.109711 & 7.232330 & 1.804259 \\
\hline $\mathrm{C}$ & 0.746668 & 5.261239 & 0.936851 \\
\hline $\mathrm{C}$ & -0.128524 & 5.790547 & -0.230988 \\
\hline $\mathrm{H}$ & 0.225475 & 6.776859 & -0.560704 \\
\hline $\mathrm{H}$ & -1.182477 & 5.880437 & 0.053494 \\
\hline $\mathrm{H}$ & -0.077088 & 5.103771 & -1.083276 \\
\hline $\mathrm{C}$ & 2.202596 & 5.122849 & 0.440177 \\
\hline $\mathrm{H}$ & 2.274943 & 4.520206 & -0.471504 \\
\hline $\mathrm{H}$ & 2.845910 & 4.670174 & 1.203989 \\
\hline $\mathrm{H}$ & 2.603023 & 6.115826 & 0.200821 \\
\hline $\mathrm{C}$ & 0.163154 & 3.937383 & 1.447337 \\
\hline
\end{tabular}




\begin{tabular}{|c|c|c|c|}
\hline $\mathrm{C}$ & 0.592257 & 2.716731 & 0.930279 \\
\hline $\mathrm{H}$ & 1.373965 & 2.714809 & 0.178263 \\
\hline $\mathrm{C}$ & 0.031775 & 1.500209 & 1.342140 \\
\hline $\mathrm{C}$ & 0.451913 & 0.182452 & 0.679280 \\
\hline $\mathrm{C}$ & 1.869278 & 0.226742 & 0.255635 \\
\hline $\mathrm{C}$ & 0.303111 & -0.955095 & 1.614242 \\
\hline $\mathrm{N}$ & 1.312267 & -5.145804 & -2.971796 \\
\hline $\mathrm{N}$ & -2.978119 & -0.291217 & 0.082549 \\
\hline $\mathrm{N}$ & -0.161474 & 1.833922 & -2.359654 \\
\hline $\mathrm{C}$ & 0.966353 & -1.519693 & -2.325689 \\
\hline $\mathrm{H}$ & 1.396934 & -0.584399 & -2.666703 \\
\hline $\mathrm{C}$ & 1.417523 & -2.710285 & -2.890560 \\
\hline $\mathrm{C}$ & 2.476441 & -2.711508 & -4.000414 \\
\hline $\mathrm{C}$ & 3.404023 & -1.478425 & -3.946074 \\
\hline $\mathrm{H}$ & 3.859385 & -1.359464 & -2.956117 \\
\hline $\mathrm{H}$ & 4.208262 & -1.594586 & -4.683181 \\
\hline $\mathrm{H}$ & 2.874723 & -0.551276 & -4.192369 \\
\hline $\mathrm{C}$ & 1.774508 & -2.747682 & -5.384263 \\
\hline $\mathrm{H}$ & 1.205505 & -1.824520 & -5.542295 \\
\hline $\mathrm{H}$ & 2.515440 & -2.835594 & -6.190514 \\
\hline $\mathrm{H}$ & 1.072594 & -3.584381 & -5.468207 \\
\hline $\mathrm{C}$ & 3.341755 & -3.976265 & -3.791246 \\
\hline $\mathrm{H}$ & 3.864925 & -3.888969 & -2.829617 \\
\hline $\mathrm{H}$ & 4.107779 & -4.047309 & -4.574185 \\
\hline $\mathrm{C}$ & 2.503219 & -5.249638 & -3.808265 \\
\hline $\mathrm{H}$ & 3.106556 & -6.094810 & -3.441959 \\
\hline $\mathrm{H}$ & 2.206566 & -5.503431 & -4.839724 \\
\hline $\mathrm{C}$ & 0.864289 & -3.949958 & -2.441405 \\
\hline $\mathrm{C}$ & 0.657964 & -6.423139 & -2.714922 \\
\hline $\mathrm{H}$ & 0.591796 & -6.970533 & -3.667837 \\
\hline $\mathrm{H}$ & 1.288011 & -7.035104 & -2.047604 \\
\hline $\mathrm{C}$ & -0.737096 & -6.250960 & -2.124794 \\
\hline $\mathrm{H}$ & -1.421169 & -5.878732 & -2.899594 \\
\hline $\mathrm{H}$ & -1.109711 & -7.232330 & -1.804259 \\
\hline $\mathrm{C}$ & -0.746668 & -5.261239 & -0.936851 \\
\hline $\mathrm{C}$ & 0.128524 & -5.790547 & 0.230988 \\
\hline $\mathrm{H}$ & -0.225475 & -6.776859 & 0.560704 \\
\hline $\mathrm{H}$ & 1.182477 & -5.880437 & -0.053494 \\
\hline $\mathrm{H}$ & 0.077088 & -5.103771 & 1.083276 \\
\hline $\mathrm{C}$ & -2.202596 & -5.122849 & -0.440177 \\
\hline
\end{tabular}




$\begin{array}{lrrr}\mathrm{H} & -2.274943 & -4.520206 & 0.471504 \\ \mathrm{H} & -2.845910 & -4.670174 & -1.203989 \\ \mathrm{H} & -2.603023 & -6.115826 & -0.200821 \\ \mathrm{C} & -0.163154 & -3.937383 & -1.447337 \\ \mathrm{C} & -0.592257 & -2.716731 & -0.930279 \\ \mathrm{H} & -1.373965 & -2.714809 & -0.178263 \\ \mathrm{C} & -0.031775 & -1.500209 & -1.342140 \\ \mathrm{C} & -0.451913 & -0.182452 & -0.679280 \\ \mathrm{C} & -1.869278 & -0.226742 & -0.255635 \\ \mathrm{C} & -0.303111 & 0.955095 & -1.614242\end{array}$

2 б-dimer $\mathrm{E}(\mathrm{RB} 97 \mathrm{D})=-1801.83313878$ a.u.

\begin{tabular}{lrrr} 
Atomic & \multicolumn{3}{c}{ Coordinates(Angstroms) } \\
type & $\mathrm{X}$ & $\mathrm{Y}$ & $\mathrm{Z}$ \\
$\mathrm{N}$ & -1.302338 & 5.105910 & 2.946689 \\
$\mathrm{~N}$ & 2.984258 & 0.175280 & -0.173490 \\
$\mathrm{~N}$ & 0.102655 & -1.962687 & 2.259267 \\
$\mathrm{C}$ & -0.918192 & 1.467216 & 2.360628 \\
$\mathrm{H}$ & -1.357461 & 0.529470 & 2.692187 \\
$\mathrm{C}$ & -1.389324 & 2.663774 & 2.900206 \\
$\mathrm{C}$ & -2.467446 & 2.683813 & 3.985890 \\
$\mathrm{C}$ & -3.375220 & 1.436130 & 3.963787 \\
$\mathrm{H}$ & -3.812100 & 1.281137 & 2.966582 \\
$\mathrm{H}$ & -4.189624 & 1.569597 & 4.692445 \\
$\mathrm{H}$ & -2.823590 & 0.527599 & 4.246008 \\
$\mathrm{C}$ & -1.782180 & 2.786159 & 5.374288 \\
$\mathrm{H}$ & -1.200862 & 1.873440 & 5.570687 \\
$\mathrm{H}$ & -2.540749 & 2.898559 & 6.166747 \\
$\mathrm{H}$ & -1.094450 & 3.642613 & 5.420816 \\
$\mathrm{C}$ & -3.349230 & 3.929401 & 3.725283 \\
$\mathrm{H}$ & -3.839374 & 3.813089 & 2.745823 \\
$\mathrm{H}$ & -4.136861 & 4.001189 & 4.490999 \\
$\mathrm{C}$ & -2.524857 & 5.215805 & 3.744069 \\
$\mathrm{H}$ & -3.122504 & 6.053314 & 3.340893 \\
$\mathrm{H}$ & -2.256861 & 5.484902 & 4.783265 \\
$\mathrm{C}$ & -0.832023 & 3.903648 & 2.445064 \\
$\mathrm{C}$ & -0.640730 & 6.387959 & 2.703624 \\
$\mathrm{H}$ & -0.585744 & 6.932073 & 3.663823 \\
$\mathrm{H}$ & -1.267328 & 6.997539 & 2.025346 \\
$\mathrm{C}$ & 0.760398 & 6.216392 & 2.119023
\end{tabular}




\begin{tabular}{|c|c|c|c|}
\hline $\mathrm{H}$ & 1.446438 & 5.848377 & 2.898662 \\
\hline $\mathrm{H}$ & 1.127267 & 7.197862 & 1.781694 \\
\hline $\mathrm{C}$ & 0.768248 & 5.213339 & 0.940650 \\
\hline $\mathrm{C}$ & -0.151767 & 5.709450 & -0.206809 \\
\hline $\mathrm{H}$ & 0.173444 & 6.705701 & -0.550209 \\
\hline $\mathrm{H}$ & -1.199727 & 5.774208 & 0.119136 \\
\hline $\mathrm{H}$ & -0.103085 & 5.008831 & -1.052467 \\
\hline $\mathrm{C}$ & 2.212683 & 5.103201 & 0.408450 \\
\hline $\mathrm{H}$ & 2.266868 & 4.480853 & -0.496052 \\
\hline $\mathrm{H}$ & 2.882636 & 4.674994 & 1.169365 \\
\hline $\mathrm{H}$ & 2.577841 & 6.107225 & 0.142549 \\
\hline $\mathrm{C}$ & 0.217714 & 3.887620 & 1.469668 \\
\hline $\mathrm{C}$ & 0.668864 & 2.664040 & 0.975704 \\
\hline $\mathrm{H}$ & 1.455886 & 2.656595 & 0.225476 \\
\hline $\mathrm{C}$ & 0.108098 & 1.445120 & 1.398487 \\
\hline $\mathrm{C}$ & 0.494546 & 0.145325 & 0.713512 \\
\hline $\mathrm{C}$ & 1.887656 & 0.143392 & 0.233726 \\
\hline $\mathrm{C}$ & 0.300135 & -1.034438 & 1.574634 \\
\hline $\mathrm{N}$ & 1.302338 & -5.105910 & -2.946689 \\
\hline $\mathrm{N}$ & -2.984258 & -0.175280 & 0.173490 \\
\hline $\mathrm{N}$ & -0.102655 & 1.962687 & -2.259267 \\
\hline $\mathrm{C}$ & 0.918192 & -1.467216 & -2.360628 \\
\hline $\mathrm{H}$ & 1.357461 & -0.529470 & -2.692187 \\
\hline $\mathrm{C}$ & 1.389324 & -2.663774 & -2.900206 \\
\hline $\mathrm{C}$ & 2.467446 & -2.683813 & -3.985890 \\
\hline $\mathrm{C}$ & 3.375220 & -1.436130 & -3.963787 \\
\hline $\mathrm{H}$ & 3.812100 & -1.281137 & -2.966582 \\
\hline $\mathrm{H}$ & 4.189624 & -1.569597 & -4.692445 \\
\hline $\mathrm{H}$ & 2.823590 & -0.527599 & -4.246008 \\
\hline $\mathrm{C}$ & 1.782180 & -2.786159 & -5.374288 \\
\hline $\mathrm{H}$ & 1.200862 & -1.873440 & -5.570687 \\
\hline $\mathrm{H}$ & 2.540749 & -2.898559 & -6.166747 \\
\hline $\mathrm{H}$ & 1.094450 & -3.642613 & -5.420816 \\
\hline $\mathrm{C}$ & 3.349230 & -3.929401 & -3.725283 \\
\hline $\mathrm{H}$ & 3.839374 & -3.813089 & -2.745823 \\
\hline $\mathrm{H}$ & 4.136861 & -4.001189 & -4.490999 \\
\hline $\mathrm{C}$ & 2.524857 & -5.215805 & -3.744069 \\
\hline $\mathrm{H}$ & 3.122504 & -6.053314 & -3.340893 \\
\hline $\mathrm{H}$ & 2.256861 & -5.484902 & -4.783265 \\
\hline $\mathrm{C}$ & 0.832023 & -3.903648 & -2.445064 \\
\hline
\end{tabular}




$\begin{array}{lrrr}\mathrm{C} & 0.640730 & -6.387959 & -2.703624 \\ \mathrm{H} & 0.585744 & -6.932073 & -3.663823 \\ \mathrm{H} & 1.267328 & -6.997539 & -2.025346 \\ \mathrm{C} & -0.760398 & -6.216392 & -2.119023 \\ \mathrm{H} & -1.446438 & -5.848377 & -2.898662 \\ \mathrm{H} & -1.127267 & -7.197862 & -1.781694 \\ \mathrm{C} & -0.768248 & -5.213339 & -0.940650 \\ \mathrm{C} & 0.151767 & -5.709450 & 0.206809 \\ \mathrm{H} & -0.173444 & -6.705701 & 0.550209 \\ \mathrm{H} & 1.199727 & -5.774208 & -0.119136 \\ \mathrm{H} & 0.103085 & -5.008831 & 1.052467 \\ \mathrm{C} & -2.212683 & -5.103201 & -0.408450 \\ \mathrm{H} & -2.266868 & -4.480853 & 0.496052 \\ \mathrm{H} & -2.882636 & -4.674994 & -1.169365 \\ \mathrm{H} & -2.577841 & -6.107225 & -0.142549 \\ \mathrm{C} & -0.217714 & -3.887620 & -1.469668 \\ \mathrm{C} & -0.668864 & -2.664040 & -0.975704 \\ \mathrm{H} & -1.455886 & -2.656595 & -0.225476 \\ \mathrm{C} & -0.108098 & -1.445120 & -1.398487 \\ \mathrm{C} & -0.494546 & -0.145325 & -0.713512 \\ \mathrm{C} & -1.887656 & -0.143392 & -0.233726 \\ \mathrm{C} & -0.300135 & 1.034438 & -1.574634\end{array}$

$2 \sigma$-dimer $\mathrm{E}(\mathrm{U} \omega \mathrm{B} 97 \mathrm{XD})=-1802.57870538$ a.u.

\begin{tabular}{lrrr} 
Atomic & \multicolumn{3}{c}{ Coordinates(Angstroms) } \\
type & $\mathrm{X}$ & \multicolumn{1}{c}{$\mathrm{Y}$} & \multicolumn{1}{c}{$\mathrm{Z}$} \\
$\mathrm{N}$ & -1.287744 & 5.086570 & 2.945055 \\
$\mathrm{~N}$ & 2.962848 & 0.248995 & -0.139234 \\
$\mathrm{~N}$ & 0.153191 & -1.875952 & 2.305038 \\
$\mathrm{C}$ & -0.940427 & 1.478691 & 2.308962 \\
$\mathrm{H}$ & -1.379031 & 0.544793 & 2.641421 \\
$\mathrm{C}$ & -1.392519 & 2.664693 & 2.864590 \\
$\mathrm{C}$ & -2.458547 & 2.679213 & 3.953036 \\
$\mathrm{C}$ & -3.362473 & 1.440463 & 3.917250 \\
$\mathrm{H}$ & -3.807622 & 1.295789 & 2.927705 \\
$\mathrm{H}$ & -4.171839 & 1.558802 & 4.645632 \\
$\mathrm{H}$ & -2.822550 & 0.527384 & 4.185365 \\
$\mathrm{C}$ & -1.773234 & 2.762683 & 5.331467 \\
$\mathrm{H}$ & -1.220399 & 1.839727 & 5.531047 \\
$\mathrm{H}$ & -2.518684 & 2.895106 & 6.124837
\end{tabular}




\begin{tabular}{|c|c|c|c|}
\hline $\mathrm{H}$ & -1.059857 & 3.590327 & 5.384277 \\
\hline $\mathrm{C}$ & -3.327457 & 3.922322 & 3.699739 \\
\hline $\mathrm{H}$ & -3.815861 & 3.815373 & 2.723978 \\
\hline $\mathrm{H}$ & -4.117444 & 3.994521 & 4.455442 \\
\hline $\mathrm{C}$ & -2.503734 & 5.196105 & 3.729732 \\
\hline $\mathrm{H}$ & -3.096971 & 6.031027 & 3.331116 \\
\hline $\mathrm{H}$ & -2.249385 & 5.461577 & 4.767562 \\
\hline $\mathrm{C}$ & -0.837311 & 3.893422 & 2.421778 \\
\hline $\mathrm{C}$ & -0.612368 & 6.351715 & 2.726159 \\
\hline $\mathrm{H}$ & -0.530297 & 6.869177 & 3.692183 \\
\hline $\mathrm{H}$ & -1.231715 & 6.992791 & 2.079629 \\
\hline $\mathrm{C}$ & 0.768234 & 6.171489 & 2.123424 \\
\hline $\mathrm{H}$ & 1.457123 & 5.780546 & 2.882044 \\
\hline $\mathrm{H}$ & 1.148590 & 7.150581 & 1.811515 \\
\hline $\mathrm{C}$ & 0.748166 & 5.202813 & 0.930152 \\
\hline $\mathrm{C}$ & -0.167705 & 5.729169 & -0.192832 \\
\hline $\mathrm{H}$ & 0.162532 & 6.720454 & -0.526009 \\
\hline $\mathrm{H}$ & -1.209901 & 5.803355 & 0.131443 \\
\hline $\mathrm{H}$ & -0.139430 & 5.048725 & -1.049146 \\
\hline $\mathrm{C}$ & 2.176632 & 5.072164 & 0.386302 \\
\hline $\mathrm{H}$ & 2.215111 & 4.484867 & -0.535714 \\
\hline $\mathrm{H}$ & 2.843670 & 4.607285 & 1.119636 \\
\hline $\mathrm{H}$ & 2.570347 & 6.066345 & 0.149542 \\
\hline $\mathrm{C}$ & 0.187684 & 3.881568 & 1.441501 \\
\hline $\mathrm{C}$ & 0.621957 & 2.668468 & 0.931418 \\
\hline $\mathrm{H}$ & 1.402916 & 2.666405 & 0.179695 \\
\hline $\mathrm{C}$ & 0.060895 & 1.461745 & 1.341259 \\
\hline $\mathrm{C}$ & 0.456234 & 0.155059 & 0.658390 \\
\hline $\mathrm{C}$ & 1.865366 & 0.190368 & 0.219922 \\
\hline $\mathrm{C}$ & 0.305472 & -0.990077 & 1.577714 \\
\hline $\mathrm{N}$ & 1.287744 & -5.086570 & -2.945055 \\
\hline $\mathrm{N}$ & -2.962848 & -0.248995 & 0.139234 \\
\hline $\mathrm{N}$ & -0.153191 & 1.875952 & -2.305038 \\
\hline $\mathrm{C}$ & 0.940427 & -1.478691 & -2.308962 \\
\hline $\mathrm{H}$ & 1.379031 & -0.544793 & -2.641421 \\
\hline $\mathrm{C}$ & 1.392519 & -2.664693 & -2.864590 \\
\hline $\mathrm{C}$ & 2.458547 & -2.679213 & -3.953036 \\
\hline $\mathrm{C}$ & 3.362473 & -1.440463 & -3.917250 \\
\hline $\mathrm{H}$ & 3.807622 & -1.295789 & -2.927705 \\
\hline $\mathrm{H}$ & 4.171839 & -1.558802 & -4.645632 \\
\hline
\end{tabular}




\begin{tabular}{lrrr}
$\mathrm{H}$ & 2.822550 & -0.527384 & -4.185365 \\
$\mathrm{C}$ & 1.773234 & -2.762683 & -5.331467 \\
$\mathrm{H}$ & 1.220399 & -1.839727 & -5.531047 \\
$\mathrm{H}$ & 2.518684 & -2.895106 & -6.124837 \\
$\mathrm{H}$ & 1.059857 & -3.590327 & -5.384277 \\
$\mathrm{C}$ & 3.327457 & -3.922322 & -3.699739 \\
$\mathrm{H}$ & 3.815861 & -3.815373 & -2.723978 \\
$\mathrm{H}$ & 4.117444 & -3.994521 & -4.455442 \\
$\mathrm{C}$ & 2.503734 & -5.196105 & -3.729732 \\
$\mathrm{H}$ & 3.096971 & -6.031027 & -3.33116 \\
$\mathrm{H}$ & 2.249385 & -5.461577 & -4.767562 \\
$\mathrm{C}$ & 0.837311 & -3.893422 & -2.421778 \\
$\mathrm{C}$ & 0.612368 & -6.351715 & -2.726159 \\
$\mathrm{H}$ & 0.530297 & -6.869177 & -3.692183 \\
$\mathrm{H}$ & 1.231715 & -6.992791 & -2.079629 \\
$\mathrm{C}$ & -0.768234 & -6.171489 & -2.123424 \\
$\mathrm{H}$ & -1.457123 & -5.780546 & -2.882044 \\
$\mathrm{H}$ & -1.148590 & -7.150581 & -1.811515 \\
$\mathrm{C}$ & -0.748166 & -5.202813 & -0.930152 \\
$\mathrm{C}$ & 0.167705 & -5.729169 & 0.192832 \\
$\mathrm{H}$ & -0.162532 & -6.720454 & 0.526009 \\
$\mathrm{H}$ & 1.209901 & -5.803355 & -0.131443 \\
$\mathrm{H}$ & 0.139430 & -5.048725 & 1.049146 \\
$\mathrm{C}$ & -2.176632 & -5.072164 & -0.386302 \\
$\mathrm{H}$ & -2.215111 & -4.484867 & 0.535714 \\
$\mathrm{H}$ & -2.843670 & -4.607285 & -1.119636 \\
$\mathrm{H}$ & -2.570347 & -6.066345 & -0.149542 \\
$\mathrm{C}$ & -0.187684 & -3.881568 & -1.441501 \\
$\mathrm{C}$ & -0.621957 & -2.668468 & -0.931418 \\
$\mathrm{H}$ & -1.402916 & -2.666405 & -0.179695 \\
$\mathrm{C}$ & -0.060895 & -1.461745 & -1.341259 \\
$\mathrm{C}$ & -0.456234 & -0.155059 & -0.658390 \\
$\mathrm{C}$ & -1.865366 & -0.190368 & -0.219922 \\
$\mathrm{C}$ & -0.305472 & 0.990077 & -1.577714 \\
& & & \\
\hline
\end{tabular}

$2 \pi$-dimer singlet $\mathrm{E}(\mathrm{UB} 98)=-1802.37879004$ a.u.; triplet $\mathrm{E}(\mathrm{UB} 98)=-1802.37843371$ a.u.

\begin{tabular}{cccc} 
Atomic & \multicolumn{3}{c}{ Coordinates(Angstroms) } \\
type & $\mathrm{X}$ & $\mathrm{Y}$ & $\mathrm{Z}$ \\
$\mathrm{N}$ & -3.805689 & -0.003433 & -0.426536 \\
$\mathrm{~N}$ & 1.787251 & -2.176940 & 3.704213
\end{tabular}




$\begin{array}{lrrr}\mathrm{N} & 1.782507 & 2.200243 & 3.697130 \\ \mathrm{C} & -2.893116 & 0.000011 & 0.585502 \\ \mathrm{C} & -2.395052 & -1.246197 & 1.121944 \\ \mathrm{C} & -1.302659 & -1.210120 & 1.965949 \\ \mathrm{C} & -0.677582 & 0.006522 & 2.359537 \\ \mathrm{C} & -1.305204 & 1.220019 & 1.960225 \\ \mathrm{C} & -2.397625 & 1.249842 & 1.116006 \\ \mathrm{C} & 0.529524 & 0.009369 & 3.130577 \\ \mathrm{C} & 1.202361 & -1.188438 & 3.471221 \\ \mathrm{C} & 1.199699 & 1.209818 & 3.467159 \\ \mathrm{C} & -3.054176 & -2.596517 & 0.778958 \\ \mathrm{C} & -4.377234 & -2.341689 & 0.020234 \\ \mathrm{H} & -4.716710 & -3.272378 & -0.450299 \\ \mathrm{H} & -5.156518 & -2.028790 & 0.728993 \\ \mathrm{C} & -4.227744 & -1.260909 & -1.045557 \\ \mathrm{H} & -3.510150 & -1.565628 & -1.825246 \\ \mathrm{H} & -5.185173 & -1.077074 & -1.543759 \\ \mathrm{C} & -4.231244 & 1.250009 & -1.051190 \\ \mathrm{H} & -5.188792 & 1.061606 & -1.547495 \\ \mathrm{H} & -3.515156 & 1.552564 & -1.833162 \\ \mathrm{C} & -4.382404 & 2.335573 & 0.009497 \\ \mathrm{H} & -4.724355 & 3.263210 & -0.465241 \\ \mathrm{H} & -5.160535 & 2.024279 & 0.720238 \\ \mathrm{C} & -3.059485 & 2.597082 & 0.766202 \\ \mathrm{H} & -0.896210 & 2.155458 & 2.332101 \\ \mathrm{H} & -0.891681 & -2.142929 & 2.342232 \\ \mathrm{~N} & 3.805517 & 0.003019 & 0.426674 \\ \mathrm{~N} & -1.787446 & 2.177128 & -3.704405 \\ \mathrm{~N} & -1.782102 & -2.200081 & -3.697112 \\ \mathrm{C} & 2.893044 & -0.000055 & -0.585461 \\ \mathrm{C} & 2.394930 & 1.246311 & -1.121510 \\ \mathrm{C} & 1.302530 & 1.210460 & -1.965539 \\ \mathrm{C} & 0.677654 & -0.006116 & -2.359672 \\ \mathrm{C} & 1.305397 & -1.219689 & -1.960811 \\ \mathrm{C} & 2.397699 & -1.249727 & -1.116472 \\ \mathrm{C} & -0.529393 & -0.008995 & -3.130793 \\ \mathrm{C} & -1.202388 & 1.188710 & -3.471505 \\ \mathrm{C} & -1.199458 & -1.209500 & -3.467367 \\ \mathrm{C} & 3.054111 & 2.596479 & -0.778036 \\ \mathrm{C} & 4.377541 & 2.341174 & -0.020113\end{array}$




\begin{tabular}{|c|c|c|c|}
\hline $\mathrm{H}$ & 4.717535 & 3.271688 & 0.450388 \\
\hline $\mathrm{H}$ & 5.156354 & 2.028183 & -0.729353 \\
\hline $\mathrm{C}$ & 4.228338 & 1.260277 & 1.045591 \\
\hline $\mathrm{H}$ & 3.511347 & 1.565097 & 1.825792 \\
\hline $\mathrm{H}$ & 5.186015 & 1.076072 & 1.543192 \\
\hline $\mathrm{C}$ & 4.230418 & -1.250662 & 1.051328 \\
\hline $\mathrm{H}$ & 5.187586 & -1.062523 & 1.548439 \\
\hline $\mathrm{H}$ & 3.513686 & -1.553337 & 1.832648 \\
\hline $\mathrm{C}$ & 4.382065 & -2.335960 & -0.009558 \\
\hline $\mathrm{H}$ & 4.723662 & -3.263784 & 0.465058 \\
\hline $\mathrm{H}$ & 5.160565 & -2.024541 & -0.719834 \\
\hline $\mathrm{C}$ & 3.059479 & -2.597078 & -0.767021 \\
\hline $\mathrm{H}$ & 0.896581 & -2.155021 & -2.333155 \\
\hline $\mathrm{H}$ & 0.891383 & 2.143374 & -2.341377 \\
\hline $\mathrm{C}$ & 2.112232 & -3.461894 & 0.103178 \\
\hline $\mathrm{H}$ & 1.165065 & -3.640941 & -0.416876 \\
\hline $\mathrm{H}$ & 2.576993 & -4.435472 & 0.304864 \\
\hline $\mathrm{H}$ & 1.888089 & -2.989609 & 1.064448 \\
\hline $\mathrm{C}$ & 3.397406 & -3.377267 & -2.063017 \\
\hline $\mathrm{H}$ & 2.496271 & -3.684629 & -2.603658 \\
\hline $\mathrm{H}$ & 4.011258 & -2.772344 & -2.740319 \\
\hline $\mathrm{H}$ & 3.957092 & -4.286837 & -1.810820 \\
\hline $\mathrm{C}$ & 2.105763 & 3.463243 & 0.089000 \\
\hline $\mathrm{H}$ & 2.567409 & 4.439776 & 0.283397 \\
\hline $\mathrm{H}$ & 1.156554 & 3.635301 & -0.429636 \\
\hline $\mathrm{H}$ & 1.885762 & 2.996257 & 1.053807 \\
\hline $\mathrm{C}$ & 3.389854 & 3.371766 & -2.077571 \\
\hline $\mathrm{H}$ & 4.003659 & 2.764779 & -2.753064 \\
\hline $\mathrm{H}$ & 2.487804 & 3.675964 & -2.618435 \\
\hline $\mathrm{H}$ & 3.948818 & 4.282946 & -1.829624 \\
\hline $\mathrm{C}$ & -3.396740 & 3.377865 & 2.062020 \\
\hline $\mathrm{H}$ & -4.009848 & 2.773096 & 2.740131 \\
\hline $\mathrm{H}$ & -2.495314 & 3.685882 & 2.601805 \\
\hline $\mathrm{H}$ & -3.956949 & 4.287080 & 1.809711 \\
\hline $\mathrm{C}$ & -2.112845 & 3.461566 & -0.104984 \\
\hline $\mathrm{H}$ & -2.577092 & 4.435671 & -0.305304 \\
\hline $\mathrm{H}$ & -1.164597 & 3.639413 & 0.413471 \\
\hline $\mathrm{H}$ & -1.890786 & 2.989702 & -1.066934 \\
\hline $\mathrm{C}$ & -3.390539 & -3.371089 & 2.078734 \\
\hline $\mathrm{H}$ & -2.488770 & -3.674729 & 2.620390 \\
\hline
\end{tabular}




$\begin{array}{lrrr}\mathrm{H} & -4.004944 & -2.763874 & 2.753481 \\ \mathrm{H} & -3.949101 & -4.282568 & 1.830968 \\ \mathrm{C} & -2.105116 & -3.463697 & -0.086939 \\ \mathrm{H} & -1.156576 & -3.635643 & 0.432975 \\ \mathrm{H} & -2.566669 & -4.440248 & -0.281497 \\ \mathrm{H} & -1.884088 & -2.997039 & -1.051663\end{array}$

$2 \pi$-dimer singlet $\mathrm{E}(\mathrm{UB} 97 \mathrm{D})=-1801.85237018$ a.u.; triplet $\mathrm{E}(\mathrm{UB} 97 \mathrm{D})=-1801.84361156$ a.u.

\begin{tabular}{lrrr} 
Atomic & \multicolumn{3}{c}{ Coordinates(Angstroms) } \\
type & $\mathrm{X}$ & $\mathrm{Y}$ & $\mathrm{Z}$ \\
$\mathrm{N}$ & 4.045283 & 0.494750 & 0.198432 \\
$\mathrm{~N}$ & -1.296912 & -3.078976 & -3.177440 \\
$\mathrm{~N}$ & -2.694900 & 1.058038 & -2.930878 \\
$\mathrm{C}$ & 2.871598 & 0.228822 & -0.442201 \\
$\mathrm{C}$ & 2.525321 & -1.141419 & -0.752107 \\
$\mathrm{C}$ & 1.270039 & -1.407582 & -1.272433 \\
$\mathrm{C}$ & 0.332188 & -0.380138 & -1.572483 \\
$\mathrm{C}$ & 0.737330 & 0.972904 & -1.333219 \\
$\mathrm{C}$ & 1.986537 & 1.300597 & -0.836316 \\
$\mathrm{C}$ & -0.900852 & -0.685236 & -2.243508 \\
$\mathrm{C}$ & -1.140723 & -1.993134 & -2.745863 \\
$\mathrm{C}$ & -1.860026 & 0.287205 & -2.612215 \\
$\mathrm{C}$ & 3.566456 & -2.279741 & -0.681562 \\
$\mathrm{C}$ & 4.951129 & -1.721765 & -0.260075 \\
$\mathrm{H}$ & 5.571118 & -2.541231 & 0.134172 \\
$\mathrm{H}$ & 5.472295 & -1.293855 & -1.132022 \\
$\mathrm{C}$ & 4.788573 & -0.622584 & 0.791284 \\
$\mathrm{H}$ & 4.232704 & -0.997471 & 1.666277 \\
$\mathrm{H}$ & 5.755897 & -0.239527 & 1.139272 \\
$\mathrm{C}$ & 4.576884 & 1.857263 & 0.342960 \\
$\mathrm{H}$ & 5.672042 & 1.792399 & 0.247306 \\
$\mathrm{H}$ & 4.344342 & 2.232214 & 1.350239 \\
$\mathrm{C}$ & 4.003377 & 2.766452 & -0.741878 \\
$\mathrm{H}$ & 4.366609 & 3.792758 & -0.582791 \\
$\mathrm{H}$ & 4.363771 & 2.428047 & -1.726999 \\
$\mathrm{C}$ & 2.455488 & 2.762656 & -0.739341 \\
$\mathrm{H}$ & 0.049057 & 1.767383 & -1.610357 \\
$\mathrm{H}$ & 1.014141 & -2.435983 & -1.522923 \\
$\mathrm{~N}$ & -4.045393 & 0.494564 & -0.198404 \\
$\mathrm{~N}$ & 2.694779 & 1.058391 & 2.931146
\end{tabular}




\begin{tabular}{|c|c|c|c|}
\hline $\mathrm{N}$ & 1.297489 & -3.078644 & 3.177290 \\
\hline $\mathrm{C}$ & -2.871624 & 0.228773 & 0.442145 \\
\hline $\mathrm{C}$ & -1.986644 & 1.300626 & 0.836188 \\
\hline $\mathrm{C}$ & -0.737416 & 0.973057 & 1.333127 \\
\hline $\mathrm{C}$ & -0.332180 & -0.379942 & 1.572450 \\
\hline $\mathrm{C}$ & -1.269932 & -1.407476 & 1.272416 \\
\hline $\mathrm{C}$ & -2.525255 & -1.141426 & 0.752122 \\
\hline $\mathrm{C}$ & 0.900865 & -0.684914 & 2.243553 \\
\hline $\mathrm{C}$ & 1.859905 & 0.287605 & 2.612355 \\
\hline $\mathrm{C}$ & 1.140900 & -1.992842 & 2.745756 \\
\hline $\mathrm{C}$ & -2.455685 & 2.762653 & 0.739146 \\
\hline $\mathrm{C}$ & -4.003577 & 2.766400 & 0.741526 \\
\hline $\mathrm{H}$ & -4.366816 & 3.792681 & 0.582292 \\
\hline $\mathrm{H}$ & -4.364065 & 2.428084 & 1.726641 \\
\hline $\mathrm{C}$ & -4.576923 & 1.857066 & -0.343284 \\
\hline $\mathrm{H}$ & -4.344166 & 2.231862 & -1.350569 \\
\hline $\mathrm{H}$ & -5.672098 & 1.792235 & -0.247848 \\
\hline $\mathrm{C}$ & -4.788513 & -0.622865 & -0.791283 \\
\hline $\mathrm{H}$ & -5.755835 & -0.239909 & -1.139389 \\
\hline $\mathrm{H}$ & -4.232517 & -0.997766 & -1.666194 \\
\hline $\mathrm{C}$ & -4.951058 & -1.721997 & 0.260138 \\
\hline $\mathrm{H}$ & -5.570926 & -2.541557 & -0.134100 \\
\hline $\mathrm{H}$ & -5.472344 & -1.294074 & 1.132004 \\
\hline $\mathrm{C}$ & -3.566351 & -2.279803 & 0.681786 \\
\hline $\mathrm{H}$ & -1.013949 & -2.435850 & 1.522933 \\
\hline $\mathrm{H}$ & -0.049201 & 1.767607 & 1.610213 \\
\hline $\mathrm{C}$ & -3.131134 & -3.370371 & -0.322480 \\
\hline $\mathrm{H}$ & -2.106956 & -3.711747 & -0.119564 \\
\hline $\mathrm{H}$ & -3.809219 & -4.234197 & -0.237698 \\
\hline $\mathrm{H}$ & -3.160761 & -3.007672 & -1.356321 \\
\hline $\mathrm{C}$ & -3.713182 & -2.922685 & 2.086369 \\
\hline $\mathrm{H}$ & -2.786763 & -3.424307 & 2.398015 \\
\hline $\mathrm{H}$ & -3.966910 & -2.161605 & 2.839860 \\
\hline $\mathrm{H}$ & -4.517693 & -3.674462 & 2.059550 \\
\hline $\mathrm{C}$ & -1.942786 & 3.450145 & -0.550675 \\
\hline $\mathrm{H}$ & -2.320326 & 4.484845 & -0.586155 \\
\hline $\mathrm{H}$ & -0.847041 & 3.489509 & -0.553284 \\
\hline $\mathrm{H}$ & -2.267711 & 2.924773 & -1.455547 \\
\hline $\mathrm{C}$ & -1.979216 & 3.582228 & 1.962417 \\
\hline $\mathrm{H}$ & -2.290885 & 3.101993 & 2.901860 \\
\hline
\end{tabular}




$\begin{array}{lrrr}\mathrm{H} & -0.886724 & 3.696820 & 1.979024 \\ \mathrm{H} & -2.420042 & 4.590140 & 1.916635 \\ \mathrm{C} & 1.978870 & 3.582201 & -1.962569 \\ \mathrm{H} & 2.290441 & 3.101958 & -2.902041 \\ \mathrm{H} & 0.886375 & 3.696778 & -1.979056 \\ \mathrm{H} & 2.419683 & 4.590121 & -1.916854 \\ \mathrm{C} & 1.942713 & 3.450155 & 0.550537 \\ \mathrm{H} & 2.320483 & 4.484767 & 0.586091 \\ \mathrm{H} & 0.846975 & 3.489744 & 0.553150 \\ \mathrm{H} & 2.267561 & 2.924653 & 1.455362 \\ \mathrm{C} & 3.713286 & -2.922960 & -2.085980 \\ \mathrm{H} & 2.786888 & -3.424709 & -2.397487 \\ \mathrm{H} & 3.966966 & -2.162066 & -2.839675 \\ \mathrm{H} & 4.517824 & -3.674700 & -2.058987 \\ \mathrm{C} & 3.131279 & -3.370087 & 0.322976 \\ \mathrm{H} & 2.107141 & -3.711592 & 0.120075 \\ \mathrm{H} & 3.809440 & -4.233880 & 0.238462 \\ \mathrm{H} & 3.160819 & -3.007119 & 1.356724\end{array}$

$2 \pi$-dimer singlet $\mathrm{E}(\mathrm{U} \omega \mathrm{B} 97 \mathrm{XD})=-1802.56279368$ a.u.; triplet $\mathrm{E}(\mathrm{U} \omega \mathrm{B} 97 \mathrm{XD})=-1802.55576292$ a.u.

\begin{tabular}{lrrr} 
Atomic & \multicolumn{3}{c}{ Coordinates(Angstroms) } \\
type & $\mathrm{X}$ & $\mathrm{Y}$ & $\mathrm{Z}$ \\
$\mathrm{N}$ & -4.057530 & 0.000026 & -0.152838 \\
$\mathrm{~N}$ & 1.984460 & -2.162898 & 3.210161 \\
$\mathrm{~N}$ & 1.984547 & 2.162283 & 3.211619 \\
$\mathrm{C}$ & -2.831870 & 0.000057 & 0.419603 \\
$\mathrm{C}$ & -2.198766 & -1.245480 & 0.768839 \\
$\mathrm{C}$ & -0.911389 & -1.215580 & 1.234580 \\
$\mathrm{C}$ & -0.219472 & 0.000067 & 1.498061 \\
$\mathrm{C}$ & -0.911320 & 1.215701 & 1.234440 \\
$\mathrm{C}$ & -2.198656 & 1.245610 & 0.768584 \\
$\mathrm{C}$ & 1.018968 & 0.000056 & 2.175830 \\
$\mathrm{C}$ & 1.561047 & -1.195237 & 2.721512 \\
$\mathrm{C}$ & 1.560954 & 1.195082 & 2.722196 \\
$\mathrm{C}$ & -2.969448 & -2.574215 & 0.775261 \\
$\mathrm{C}$ & -4.443047 & -2.328505 & 0.398618 \\
$\mathrm{H}$ & -4.890361 & -3.264218 & 0.047754 \\
$\mathrm{H}$ & -5.004223 & -2.010380 & 1.285432 \\
$\mathrm{C}$ & -4.595510 & -1.253784 & -0.661996
\end{tabular}




\begin{tabular}{|c|c|c|c|}
\hline $\mathrm{H}$ & -4.084984 & -1.534468 & -1.592736 \\
\hline $\mathrm{H}$ & -5.650862 & -1.092828 & -0.894574 \\
\hline $\mathrm{C}$ & -4.595418 & 1.253737 & -0.662347 \\
\hline $\mathrm{H}$ & -5.650808 & 1.092829 & -0.894790 \\
\hline $\mathrm{H}$ & -4.084919 & 1.534098 & -1.593197 \\
\hline $\mathrm{C}$ & -4.442780 & 2.328751 & 0.397929 \\
\hline $\mathrm{H}$ & -4.890016 & 3.264402 & 0.046800 \\
\hline $\mathrm{H}$ & -5.003934 & 2.010959 & 1.284877 \\
\hline $\mathrm{C}$ & -2.969161 & 2.574433 & 0.774442 \\
\hline $\mathrm{H}$ & -0.420523 & 2.146193 & 1.503150 \\
\hline $\mathrm{H}$ & -0.420635 & -2.146069 & 1.503378 \\
\hline $\mathrm{N}$ & 4.057569 & 0.000488 & 0.152770 \\
\hline $\mathrm{N}$ & -1.985183 & 2.161382 & -3.211292 \\
\hline $\mathrm{N}$ & -1.983881 & -2.163813 & -3.210346 \\
\hline $\mathrm{C}$ & 2.831898 & 0.000205 & -0.419648 \\
\hline $\mathrm{C}$ & 2.198469 & 1.245596 & -0.768852 \\
\hline $\mathrm{C}$ & 0.911120 & 1.215388 & -1.234644 \\
\hline $\mathrm{C}$ & 0.219465 & -0.000413 & -1.498048 \\
\hline $\mathrm{C}$ & 0.911602 & -1.215887 & -1.234391 \\
\hline $\mathrm{C}$ & 2.198992 & -1.245485 & -0.768641 \\
\hline $\mathrm{C}$ & -1.018983 & -0.000685 & -2.175778 \\
\hline $\mathrm{C}$ & -1.561359 & 1.194217 & -2.721999 \\
\hline $\mathrm{C}$ & -1.560712 & -1.196081 & -2.721621 \\
\hline $\mathrm{C}$ & 2.968722 & 2.574557 & -0.774918 \\
\hline $\mathrm{C}$ & 4.442358 & 2.329241 & -0.398208 \\
\hline $\mathrm{H}$ & 4.889358 & 3.265021 & -0.047123 \\
\hline $\mathrm{H}$ & 5.003689 & 2.011488 & -1.285059 \\
\hline $\mathrm{C}$ & 4.595170 & 1.254352 & 0.662173 \\
\hline $\mathrm{H}$ & 4.084601 & 1.534670 & 1.593007 \\
\hline $\mathrm{H}$ & 5.650595 & 1.093703 & 0.894641 \\
\hline $\mathrm{C}$ & 4.595770 & -1.253150 & 0.662154 \\
\hline $\mathrm{H}$ & 5.651099 & -1.091967 & 0.894675 \\
\hline $\mathrm{H}$ & 4.085307 & -1.533751 & 1.592943 \\
\hline $\mathrm{C}$ & 4.443455 & -2.328091 & -0.398257 \\
\hline $\mathrm{H}$ & 4.890912 & -3.263669 & -0.047218 \\
\hline $\mathrm{H}$ & 5.004574 & -2.010060 & -1.285143 \\
\hline $\mathrm{C}$ & 2.969883 & -2.574089 & -0.774823 \\
\hline $\mathrm{H}$ & 0.421009 & -2.146508 & -1.503028 \\
\hline $\mathrm{H}$ & 0.420164 & 2.145750 & -1.503509 \\
\hline $\mathrm{C}$ & 2.343551 & -3.595697 & 0.188023 \\
\hline
\end{tabular}




$\begin{array}{lrrr}\mathrm{H} & 1.272990 & -3.718086 & -0.007814 \\ \mathrm{H} & 2.820168 & -4.571811 & 0.045935 \\ \mathrm{H} & 2.461676 & -3.307014 & 1.233423 \\ \mathrm{C} & 2.939751 & -3.159459 & -2.201357 \\ \mathrm{H} & 1.933991 & -3.477884 & -2.489639 \\ \mathrm{H} & 3.287959 & -2.426854 & -2.936237 \\ \mathrm{H} & 3.593696 & -4.037080 & -2.252820 \\ \mathrm{C} & 2.341631 & 3.595544 & 0.188073 \\ \mathrm{H} & 2.818635 & 4.571682 & 0.047493 \\ \mathrm{H} & 1.271341 & 3.718347 & -0.008969 \\ \mathrm{H} & 2.458369 & 3.305635 & 1.233263 \\ \mathrm{C} & 2.938499 & 3.160194 & -2.201320 \\ \mathrm{H} & 3.287294 & 2.427956 & -2.936289 \\ \mathrm{H} & 1.932611 & 3.478080 & -2.489751 \\ \mathrm{H} & 3.591924 & 4.038223 & -2.252476 \\ \mathrm{C} & -2.938904 & 3.160289 & 2.200776 \\ \mathrm{H} & -3.287482 & 2.428087 & 2.935880 \\ \mathrm{H} & -1.933046 & 3.478413 & 2.489045 \\ \mathrm{H} & -3.592497 & 4.038195 & 2.251860 \\ \mathrm{C} & -2.342378 & 3.595462 & -0.188684 \\ \mathrm{H} & -2.819291 & 4.571600 & -0.047748 \\ \mathrm{H} & -1.272008 & 3.718216 & 0.007979 \\ \mathrm{H} & -2.459501 & 3.305869 & -1.233931 \\ \mathrm{C} & -2.939232 & -3.159369 & 2.201861 \\ \mathrm{H} & -1.933403 & -3.477487 & 2.490244 \\ \mathrm{H} & -3.287672 & -2.426759 & 2.936627 \\ \mathrm{H} & -3.592946 & -4.037159 & 2.253413 \\ \mathrm{C} & -2.342905 & -3.595788 & -0.187491 \\ \mathrm{H} & -1.272368 & -3.718100 & 0.008544 \\ \mathrm{H} & -2.819488 & -4.571934 & -0.045585 \\ \mathrm{H} & -2.460825 & -3.306864 & -1.232829\end{array}$

Derivative of 1 lacking methyl groups--pimer

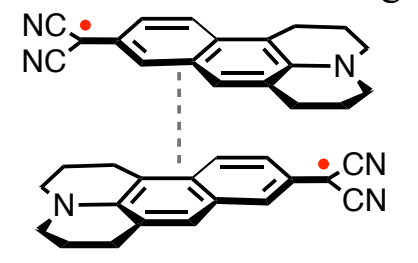

$\omega$ B97XD $\quad-1795.28296318$ a.u.

$\mathrm{N} \quad-1.94407800 \quad-3.97687900 \quad-1.02288400$ 


\begin{tabular}{|c|c|c|c|}
\hline $\mathrm{N}$ & -3.53319900 & -0.18434200 & -2.55866300 \\
\hline $\mathrm{C}$ & 2.36327200 & 0.21180100 & -1.15329000 \\
\hline $\mathrm{C}$ & 2.10625500 & -1.17788500 & -0.86389100 \\
\hline $\mathrm{C}$ & 0.83495600 & -1.69792600 & -0.96167300 \\
\hline $\mathrm{C}$ & -0.26672000 & -0.91017100 & -1.40292800 \\
\hline $\mathrm{C}$ & -0.00798400 & 0.47591300 & -1.71290700 \\
\hline $\mathrm{C}$ & 1.23868300 & 0.99737900 & -1.60304500 \\
\hline $\mathrm{C}$ & -1.53566400 & -1.47351100 & -1.53890000 \\
\hline $\mathrm{C}$ & -1.77052700 & -2.85170900 & -1.25585300 \\
\hline $\mathrm{C}$ & -2.63584400 & -0.75339500 & -2.08780200 \\
\hline $\mathrm{H}$ & -0.83306100 & 1.09811900 & -2.04427700 \\
\hline $\mathrm{H}$ & 0.67163400 & -2.74699300 & -0.73409900 \\
\hline $\mathrm{N}$ & 1.66222500 & 3.36928200 & 1.10728700 \\
\hline $\mathrm{N}$ & 3.57033200 & -0.26166200 & 2.48737700 \\
\hline $\mathrm{C}$ & -2.38362900 & -1.04547300 & 1.36494600 \\
\hline $\mathrm{C}$ & -2.27163000 & 0.35010600 & 1.07356700 \\
\hline $\mathrm{C}$ & -1.00648600 & 0.94513500 & 1.18470300 \\
\hline $\mathrm{C}$ & 0.12798600 & 0.22378000 & 1.59108600 \\
\hline $\mathrm{C}$ & -0.01659900 & -1.17349700 & 1.89402800 \\
\hline $\mathrm{C}$ & -1.24097900 & -1.76855800 & 1.80068000 \\
\hline $\mathrm{C}$ & 1.39700100 & 0.85869100 & 1.66584000 \\
\hline $\mathrm{C}$ & 1.54274400 & 2.24087600 & 1.36928900 \\
\hline $\mathrm{C}$ & 2.57062500 & 0.22071700 & 2.13127000 \\
\hline $\mathrm{H}$ & 0.85302900 & -1.74003800 & 2.21241800 \\
\hline $\mathrm{H}$ & -0.88200100 & 1.99730300 & 0.95839400 \\
\hline $\mathrm{C}$ & 3.65565900 & 0.73454000 & -1.02900100 \\
\hline $\mathrm{C}$ & 4.45026600 & -1.50968400 & -0.30089600 \\
\hline $\mathrm{C}$ & 4.70292300 & -0.10250700 & -0.56477800 \\
\hline $\mathrm{C}$ & 3.19830600 & -1.99947900 & -0.45240200 \\
\hline $\mathrm{C}$ & -3.64481500 & -1.66644700 & 1.21606300 \\
\hline $\mathrm{C}$ & -3.41906600 & 1.08042600 & 0.63456100 \\
\hline $\mathrm{C}$ & -4.62567800 & 0.41999600 & 0.43616100 \\
\hline $\mathrm{C}$ & -4.73313000 & -0.98814300 & 0.74391000 \\
\hline $\mathrm{H}$ & -1.35154500 & -2.82662100 & 2.01990100 \\
\hline $\mathrm{H}$ & 1.38296300 & 2.04401600 & -1.83874100 \\
\hline $\mathrm{H}$ & 3.00332300 & -3.04713300 & -0.23805800 \\
\hline $\mathrm{C}$ & 5.57960900 & -2.39673000 & 0.15339200 \\
\hline $\mathrm{C}$ & 3.92643600 & 2.19519700 & -1.32096900 \\
\hline $\mathrm{C}$ & 6.57305600 & -1.59001100 & 0.97605800 \\
\hline $\mathrm{H}$ & 6.08513300 & -1.21650500 & 1.88184500 \\
\hline $\mathrm{H}$ & 7.43044900 & -2.20110100 & 1.27198000 \\
\hline $\mathrm{C}$ & 7.06015100 & -0.42264800 & 0.13800900 \\
\hline $\mathrm{H}$ & 7.69346900 & 0.23487100 & 0.74102900 \\
\hline $\mathrm{H}$ & 7.67114400 & -0.78245000 & -0.70423800 \\
\hline $\mathrm{C}$ & 6.30580100 & 1.74148800 & -0.78324400 \\
\hline $\mathrm{H}$ & 6.60273300 & 1.74059800 & -1.84295200 \\
\hline
\end{tabular}




$\begin{array}{lrrr}\mathrm{H} & 7.17760100 & 2.04990300 & -0.20056900 \\ \mathrm{~N} & 5.95537100 & 0.38688700 & -0.37182400 \\ \mathrm{C} & 5.14556900 & 2.69217200 & -0.55886100 \\ \mathrm{H} & 4.91886900 & 2.74504000 & 0.51134000 \\ \mathrm{H} & 5.42200200 & 3.69714200 & -0.88902600 \\ \mathrm{~N} & -5.74139000 & 1.09136500 & -0.05143400 \\ \mathrm{C} & -3.27033800 & 2.55261200 & 0.32677700 \\ \mathrm{C} & -4.61518700 & 3.23951600 & 0.11520100 \\ \mathrm{H} & -4.47553500 & 4.21062100 & -0.36911100 \\ \mathrm{H} & -5.10450100 & 3.41597800 & 1.07937500 \\ \mathrm{C} & -5.51281500 & 2.35482500 & -0.73139500 \\ \mathrm{H} & -6.48535300 & 2.83161000 & -0.88762600 \\ \mathrm{H} & -5.05807800 & 2.18124400 & -1.72108200 \\ \mathrm{C} & -6.01882700 & -1.73849500 & 0.48708300 \\ \mathrm{C} & -7.21734800 & -0.80350900 & 0.40367200 \\ \mathrm{H} & -7.45276000 & -0.39089800 & 1.39121200 \\ \mathrm{H} & -8.10057500 & -1.33963600 & 0.04418800 \\ \mathrm{C} & -6.88060500 & 0.33427300 & -0.54412900 \\ \mathrm{H} & -6.68232600 & -0.06271000 & -1.55459500 \\ \mathrm{H} & -7.72372100 & 1.02794600 & -0.62281800 \\ \mathrm{H} & -3.73241200 & -2.72895900 & 1.42999100 \\ \mathrm{H} & 4.07970500 & 2.33562700 & -2.40001700 \\ \mathrm{H} & 3.06904100 & 2.80552500 & -1.03410000 \\ \mathrm{H} & 6.08877500 & -2.82345000 & -0.72187100 \\ \mathrm{H} & 5.17520800 & -3.23296300 & 0.72958700 \\ \mathrm{H} & -5.91712900 & -2.27388500 & -0.46652100 \\ \mathrm{H} & -6.15998700 & -2.50017700 & 1.26039900 \\ \mathrm{H} & -2.64996300 & 2.67405100 & -0.57304800 \\ \mathrm{H} & -2.73353100 & 3.05339600 & 1.14010200 \\ & & & \\ & & & \\ & & & \end{array}$

$\begin{array}{lrrr} & \\ \omega B 97 X D & -1795.29839027 \text { a.u. } & \\ N & 0.47678800 & -2.87066400 & 2.02191000 \\ N & 0.51515100 & 1.41436200 & 1.99784000 \\ N & -8.13761200 & 0.59539000 & -0.12975900 \\ N & -0.47682200 & -2.87041700 & -2.02226200 \\ N & -0.51501600 & 1.41466000 & -1.99748700 \\ N & -0.34700100 & -0.72750300 & 0.73561300 \\ \mathrm{C} & 0.12917100 & -1.91922900 & 1.46477300 \\ \mathrm{C} & 0.14729700 & 0.46229200 & 1.45492000 \\ \mathrm{C} & -1.87750500 & -0.72041400 & 0.65551500 \\ \mathrm{C} & -2.58619500 & -1.93999000 & 0.54009400 \\ \mathrm{C} & -2.05412400 & -2.88548600 & 0.56164500 \\ \mathrm{H} & & \end{array}$




\begin{tabular}{|c|c|c|c|}
\hline $\mathrm{C}$ & -3.94962800 & -1.94000600 & 0.39589500 \\
\hline $\mathrm{H}$ & -4.45328500 & -2.89401000 & 0.29521400 \\
\hline $\mathrm{C}$ & -4.69844600 & -0.72988100 & 0.35687100 \\
\hline $\mathrm{C}$ & -6.11141700 & -0.70488500 & 0.18663900 \\
\hline $\mathrm{C}$ & -6.89573400 & -1.99623000 & 0.12554800 \\
\hline $\mathrm{C}$ & -8.35913400 & -1.76506400 & 0.48820700 \\
\hline $\mathrm{H}$ & -8.95031500 & -2.66653000 & 0.30207100 \\
\hline $\mathrm{H}$ & -8.44272600 & -1.52870400 & 1.55512700 \\
\hline $\mathrm{C}$ & -8.91965600 & -0.61128400 & -0.32178000 \\
\hline $\mathrm{H}$ & -8.95042200 & -0.87980600 & -1.39210200 \\
\hline $\mathrm{H}$ & -9.94984400 & -0.39665000 & -0.01448100 \\
\hline $\mathrm{C}$ & -6.77289300 & 0.52038600 & 0.08827000 \\
\hline $\mathrm{C}$ & -8.81854800 & 1.81484000 & -0.55144000 \\
\hline $\mathrm{H}$ & -9.51333200 & 2.13070200 & 0.24134900 \\
\hline $\mathrm{H}$ & -9.42839000 & 1.57943600 & -1.43443000 \\
\hline $\mathrm{C}$ & -7.86330100 & 2.95132800 & -0.88200000 \\
\hline $\mathrm{H}$ & -8.43090600 & 3.88244800 & -0.96825400 \\
\hline $\mathrm{H}$ & -7.37920600 & 2.76821700 & -1.84795900 \\
\hline $\mathrm{C}$ & -6.79781800 & 3.04410300 & 0.20522600 \\
\hline $\mathrm{C}$ & -6.03854100 & 1.74643500 & 0.25451800 \\
\hline $\mathrm{C}$ & -4.68570000 & 1.71162700 & 0.42786200 \\
\hline $\mathrm{H}$ & -4.13681700 & 2.64451200 & 0.53286200 \\
\hline $\mathrm{C}$ & -3.97189100 & 0.48771500 & 0.47251000 \\
\hline $\mathrm{C}$ & -2.56953300 & 0.46841500 & 0.62698400 \\
\hline $\mathrm{H}$ & -2.05049700 & 1.41854500 & 0.71748700 \\
\hline $\mathrm{C}$ & 0.34699200 & -0.72746800 & -0.73562100 \\
\hline $\mathrm{C}$ & -0.12918700 & -1.91915800 & -1.46483700 \\
\hline $\mathrm{C}$ & -0.14729700 & 0.46236300 & -1.45487400 \\
\hline $\mathrm{C}$ & 1.87749700 & -0.72039200 & -0.65552000 \\
\hline $\mathrm{C}$ & 2.56953100 & 0.46843300 & -0.62699400 \\
\hline $\mathrm{H}$ & 2.05050100 & 1.41856500 & -0.71750600 \\
\hline $\mathrm{C}$ & 3.97188800 & 0.48772600 & -0.47251600 \\
\hline $\mathrm{C}$ & 4.68570500 & 1.71163400 & -0.42787500 \\
\hline $\mathrm{H}$ & 4.13682800 & 2.64452200 & -0.53288200 \\
\hline $\mathrm{C}$ & 6.03854600 & 1.74643600 & -0.25452600 \\
\hline $\mathrm{C}$ & 6.79783000 & 3.04409900 & -0.20524100 \\
\hline $\mathrm{C}$ & 6.77289000 & 0.52038300 & -0.08826800 \\
\hline $\mathrm{C}$ & 8.91964600 & -0.61129700 & 0.32179400 \\
\hline $\mathrm{H}$ & 8.95040800 & -0.87981200 & 1.39211800 \\
\hline $\mathrm{H}$ & 9.94983500 & -0.39667100 & 0.01449600 \\
\hline $\mathrm{C}$ & 8.35911800 & -1.76507900 & -0.48818700 \\
\hline $\mathrm{H}$ & 8.95029400 & -2.66654700 & -0.30204300 \\
\hline $\mathrm{H}$ & 8.44271500 & -1.52872600 & -1.55510800 \\
\hline $\mathrm{C}$ & 6.89571600 & -1.99623400 & -0.12552900 \\
\hline $\mathrm{C}$ & 6.11140700 & -0.70488400 & -0.18663200 \\
\hline $\mathrm{C}$ & 4.69843700 & -0.72987400 & -0.35686700 \\
\hline
\end{tabular}




$\begin{array}{lrrr}\mathrm{C} & 3.94961100 & -1.93999500 & -0.39588500 \\ \mathrm{H} & 4.45326300 & -2.89400100 & -0.29519600 \\ \mathrm{C} & 2.58617900 & -1.93997200 & -0.54008900 \\ \mathrm{H} & 2.05410200 & -2.88546500 & -0.56163300 \\ \mathrm{~N} & 8.13760800 & 0.59538100 & 0.12976400 \\ \mathrm{C} & 7.86331000 & 2.95132500 & 0.88198800 \\ \mathrm{H} & 8.43092000 & 3.88244300 & 0.96823700 \\ \mathrm{C} & 8.81855100 & 1.81482900 & 0.55143700 \\ \mathrm{H} & 9.51333800 & 2.13068100 & -0.24135300 \\ \mathrm{H} & 9.42839100 & 1.57942800 & 1.43443000 \\ \mathrm{H} & 7.37921200 & 2.76822300 & 1.84794700 \\ \mathrm{H} & -7.28332900 & 3.23047900 & 1.17322600 \\ \mathrm{H} & -6.11030000 & 3.87458700 & 0.02205300 \\ \mathrm{H} & -6.47197700 & -2.72546000 & 0.82287500 \\ \mathrm{H} & -6.82380100 & -2.44434100 & -0.87520100 \\ \mathrm{H} & 6.82377800 & -2.44433800 & 0.87522200 \\ \mathrm{H} & 6.47195700 & -2.72546600 & -0.82285200 \\ \mathrm{H} & 6.11031600 & 3.87458800 & -0.02207500 \\ \mathrm{H} & 7.28334400 & 3.23046600 & -1.17324100\end{array}$

